\title{
Lithium and Dilithium Guanidinates, a Starter Kit for Metal Complexes Containing Various Mono- and Dianionic Ligands
}

\author{
Tomáš Chlupatý, Jana Nevoralová, Zdeňka Růžičková, Aleš Růžička*
}

Department of General and Inorganic Chemistry, Faculty of Chemical Technology, University of Pardubice, Studentská 573, CZ-532 10, Pardubice, Czech Republic, Fax: +420-466037068,

*E-mail: ales.ruzicka@upce.cz

\section{TABLE OF CONTENTS}

Molecular structure of $\mathbf{2}$ - Figure $\mathbf{S 1}$ page S3

Molecular structure of $\mathbf{2 a}$ - Figure S2. .pages S3 - S4

Molecular structure of $\mathbf{3}$ - Figure S3. page $\mathrm{S} 4$

Molecular structure of 4 - Figure S4. page S5

Molecular structure of 4 a - Figure S5 page $\mathrm{S} 6$

Molecular structure of 5 - Figure S6. page 57

Molecular structure of $6 .\left[\mathrm{Et}_{2} \mathrm{O}\right]_{2}$ - Figure S7. page S7

Molecular structure of $\left[\text { 7. } \mathrm{Et}_{2} \mathrm{O}\right]_{2}$ - Figure $\mathbf{S 8}$. page S8

Molecular structure of 8- Figure $\mathbf{S 9 .}$ page $\mathrm{S} 8$

Molecular structure of 9 - Figure S10. page 59

Molecular structure of $\mathbf{1 0}$ - Figure S11. pages S9 - S10

Molecular structure of $\mathbf{1 1}$ - Figure S12. ..page S10

Molecular structure of $[12]_{2}$ - Figure S13. page S11

Molecular structure of 14 - Figure S14. page S11

Proposed THF solvation of $\mathbf{1 3}$ and $\mathbf{1 4}$ - Scheme S1. .page S12

General methods. .pages $\mathrm{S} 12-\mathrm{S} 13$

Crystallographic data for 2 - 5, 6. $\left[\mathrm{Et}_{2} \mathrm{O}\right]_{2},\left[7 . \mathrm{Et}_{2} \mathrm{O}\right]_{2}, 8-11,[12]_{2}$ and 14 - Table S1 pages S14 - S15 Lithium [2,6-di(propan-2-yl)phenyl]azanide (1)..... page S16

Lithium trimethylsilyl[2,6-di(propan-2-yl)phenyl]azanide (6). page S16

Lithium 2-[(dimethylamino)methyl]phenylazanide (7). .page S16 
General procedure of preparation of lithium

$N, N^{\prime}$-disubstituted-\{ $N^{\prime \prime}$-[2,6-di(propan-2-yl)phenyl]\}guanidinates (2 - 3)

pages S16 - S17

General procedure of preparation of lithium

$N, N^{\prime}$-disubstituted-\{N')-[2-[(dimethylamino)methyl]phenyl $\}$ guanidinates (8 - 9) page S17

General procedure of preparation of lithium $N, N^{\prime}$-disubstituted-

$\left\{N^{\prime \prime}\right.$-trimethylsilyl-[2-[(dimethylamino)methyl]phenyl\}guanidinates (13 - 14) page S17

Preparation of $\mathbf{2}$ pages S17 - S18

Preparation of $\mathbf{3}$ page S18

Preparation of 4 page S18

Preparation of $\mathbf{4 a}$ pages S18 - S19

Preparation of $\mathbf{5}$ page S19

Preparation of 6. $\left[\mathrm{Et}_{\mathbf{2}} \mathrm{O}\right]_{2}$ page S19

Preparation of $\left[\text { 7. } \mathrm{Et}_{\mathbf{2}} \mathrm{O}\right]_{2}$ page S19

Preparation of $\mathbf{8}$ pages S19 - S20

Preparation of $\mathbf{9}$ page S20

Preparation of $\mathbf{1 0}$ page S21

Preparation of $\mathbf{1 1}$ page S21

Preparation of [12] page S21

Preparation of 13 pages S21 - S22

Preparation of 14 page S22

References. .pages S22 - S23

Scanned NMR spectra of titled compound pages S24 - S60 


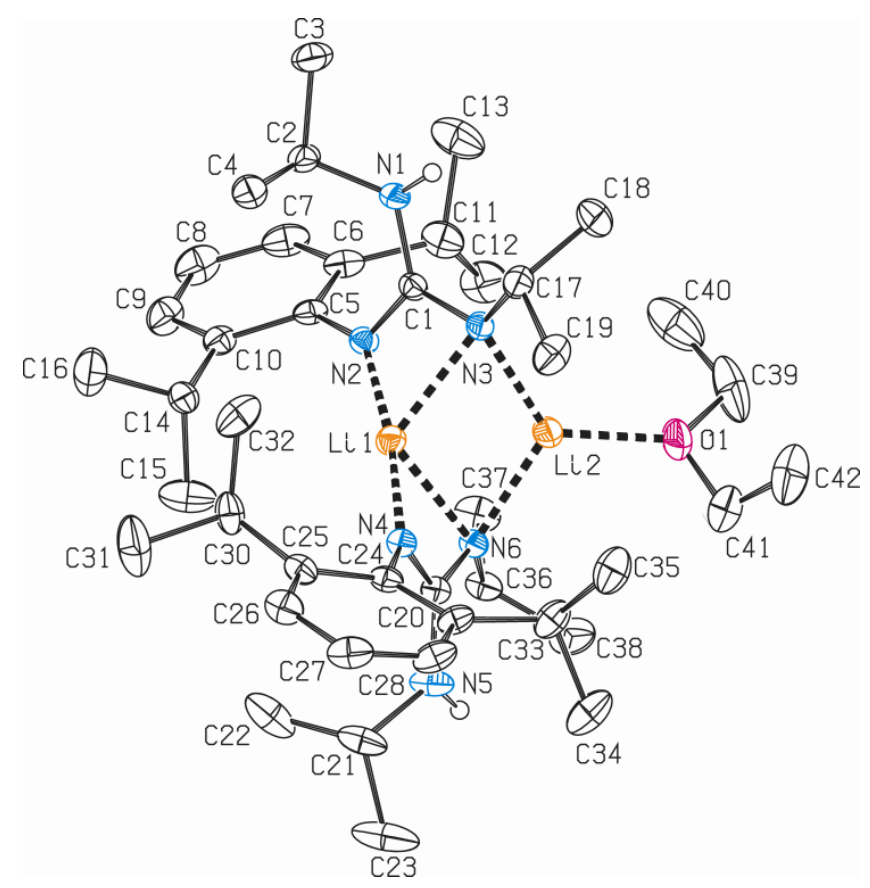

Figure S1 Molecular structure of 2 (ORTEP view, 40\% probability level). Hydrogen atoms are omitted for clarity. Selected interatomic distances [Å] and angles [ ${ }^{\circ}$ ]: Li1-N2 1.964(4), Li1-N4 1.975(4), Li1-N3 2.181(4), Li1-N6 2.120(4), Li2-N3 2.004(4), Li2-N6 2.024(4), Li2-O1 1.940(4), C1-N1 1.388(2), C1-N2 1.335(2), C1-N3 1.340(3), N1-C2 1.470(3), C20-N4 1.332(2), C20-N5 1.389(2), C20-N6 1.339(2), N5C21 1.464(3); N2-Li1-N3 65.95(12), N4-Li1-N6 67.17(12), N2-Li1-N4 172.8(2), N3-Li1-N6 104.66(15), N3-Li2-N6 115.42(18), N3-Li2-O1 124.72(19), N2-C1-N3 115.58(17), N4-C20-N6 116.28(17), C1-N1-C2 123.98(16), C20-N5-C21 126.98(18).

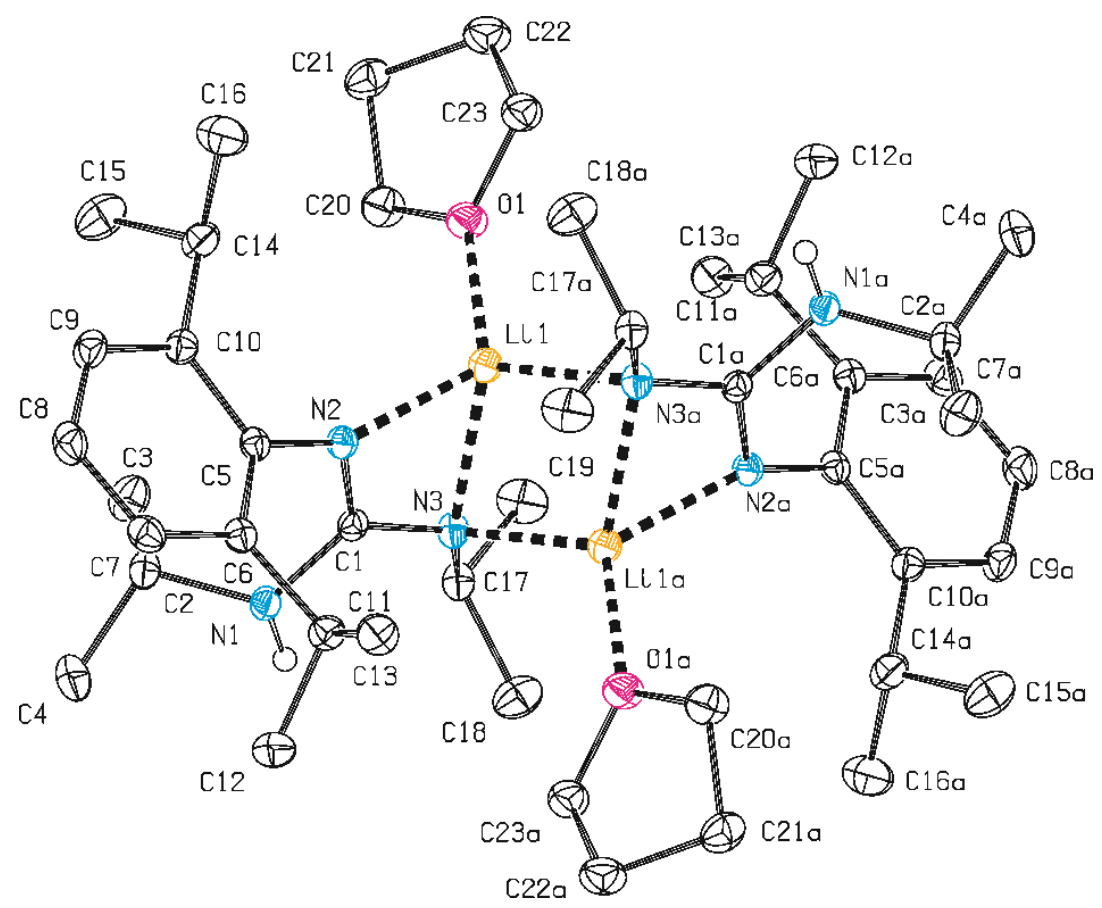


Figure S2 Molecular structure of 2a (ORTEP view, 40\% probability level). Hydrogen atoms are omitted for clarity. Selected interatomic distances [Å] and angles [ ${ }^{\circ}$ ]: N2-C1 1.3317(17), N2-C5 1.4043(16), N2Li1 2.051(3), O1-C23 1.4354(18), O1-Li1 1.995(2), C1-N3 1.3388(16), C1-N1 1.3919(17), C1-Li1 2.419(3), N3-C17 1.4663(16), N3-Li1a 2.064(2), N3-Li1 2.108(3), N2-C1-N3 116.76(11), N2-C1-N1 122.27(11), N3-C1-N1 120.97(12), O1-Li1-N2 118.89(12), O1-Li1-N3a 115.85(12), N2-Li1-N3a 117.36(11), O1-Li1-N3 123.88(12), N2-Li1-N3 66.28(8), N3-Li1-N3a 105.02(10), O1-Li1-C1 123.54(11), N3-Li1-C1a 120.22(11), O1-Li1-Li1a 144.80(17), N2-Li1-Li1a 92.28(13), N3a-Li1-Li1a 53.29(9).

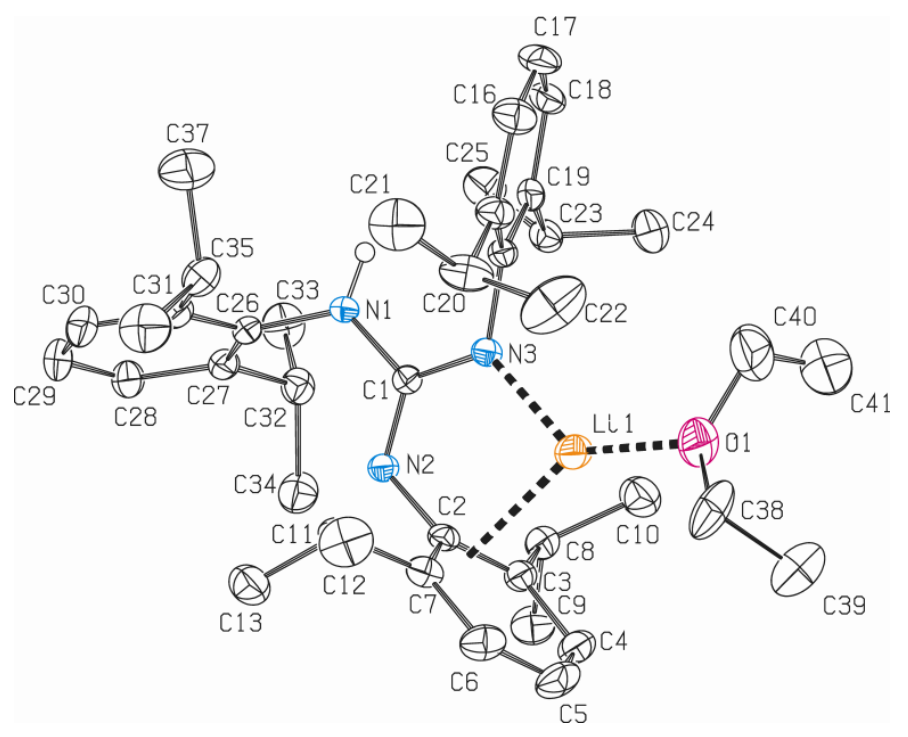

Figure S3 Molecular structure of 3 (ORTEP view, 40\% probability level). Disorder of diethylether molecule and hydrogen atoms are omitted for clarity. Selected interatomic distances $[\AA \AA]$ and angles []: Li1-N2 3.048(6), Li1-N3 1.937(5), Li1-Cg(C3, C2, C7) 2.301(4), Li1-O1 1.880(5), C1-N1 1.400(3), C1N2 1.307(3), C1-N3 1.349(3), N1-C26 1.429(3); N3-Li1-Cg(C3, C2, C7) 88.36(5), N3-Li1-O1 129.5(3), N2-C1-N3 125.0(2), C1-N1-C26 122.05(18). 


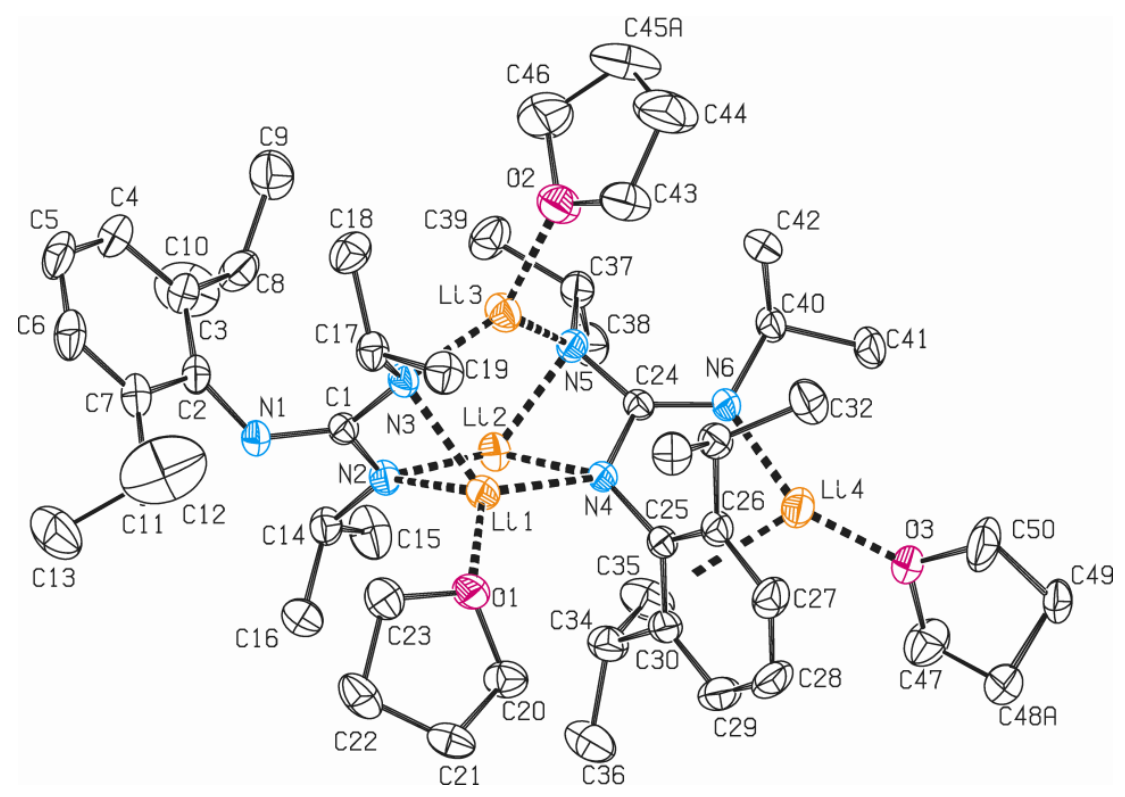

Figure S4 Molecular structure of 4 (ORTEP view, 50\% probability level). Hydrogen atoms and disordered THF parts are omitted for clarity. Selected interatomic distances [ $[\AA]$ and angles $\left[^{\circ}\right]$ : Li1-N2 2.046(4), Li1-N3 2.095(4), Li1-N4 2.138(4), Li1-O1 1.955(4), Li2-N2 1.916(4), Li2-N4 2.026(4), Li2-N5 1.978(4), Li3-N3 2.001(4), Li3-N5 2.007(4), Li3-O2 2.024(4), Li4-N6 1.922(4), Li4-Cg(C26, C25, C30) 2.292(5), Li4-O3 1.872(4), C1-N1 1.336(2), C1-N2 1.370(3), C1-N3 1.387(3), C24-N4 1.393(2), N5-C24 1.374(2), N6-C24 1.338(2); N2-Li1-N3 67.14(12), N2-Li1-N4 103.17(17), N3-Li1-N4 118.25(17), Li1-N2Li2 73.88(16), Li1-N4-Li2 69.77(15), N2-Li2-N4 112.56(19), N2-Li2-N5 129.6(2), N4-Li2-N5 68.82(13), Li1-N3-Li3 85.10(15), N3-Li3-N5 127.7(2), Li2-N5-Li3 87.22(16), N6-Li4-Cg(C26, C25, C30) 89.41(6), O3Li4-N6 146.5(2), N1-C1-N2 119.69(18), N1-C1-N3 127.63(19), N4-C24-N6 118.54(17), N4-C24-N5 109.69(16). 

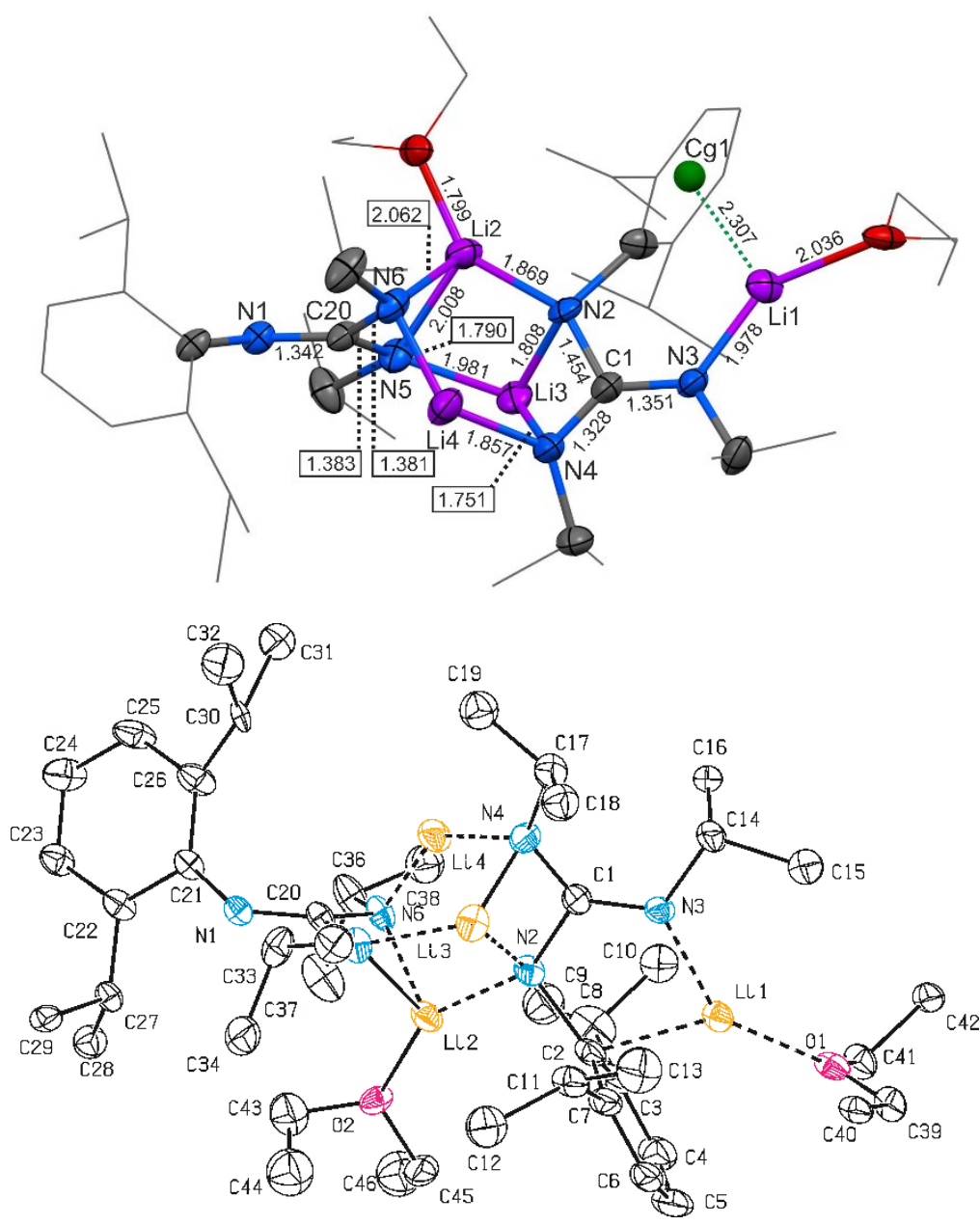

Figure S5 Molecular structure of 4a (schematic and ORTEP views, 30\% probability level). Hydrogen atoms and disordered parts are omitted for clarity. Selected interatomic distances $[\AA \AA]$ and angles $\left[^{\circ}\right]$ : Li1-N3 1.98(4), Li1-O1 2.04(3), Li1-C2 2.16(3), Li1-C3 2.44(3), Li1-C7 2.44(3), Li2-O2 1.80(2), Li2-N2 1.87(3), Li2-N5 2.01(3), Li2-N6 2.06(3), Li2-Li3 2.44(4), Li2-Li4 2.93(3), Li3-N4 1.74(5), Li3-N2 1.80(4), Li3-C1 1.95(4), Li3-N5 1.99(3), Li3-Li4 2.36(4), Li4-N6 1.79(2), Li4-N4 1.86(3), N3-Li1 O1 142.2(19), O2Li2-N2 144.0(17), O2-Li2-N5 117.5(14), N2-Li2-N5 98.0(14), O2-Li2-N6 102.9(12), N2-Li2-N6 95.9(12), N5-Li2-N6 66.9(9), N2-Li3-N5 101.1(15), Li4-Li3-Li2 75.3(11), N6-Li4-N4 133.6(15), N6-Li4-N5 60.6(8), N4-Li4-N5 94.7(13) 


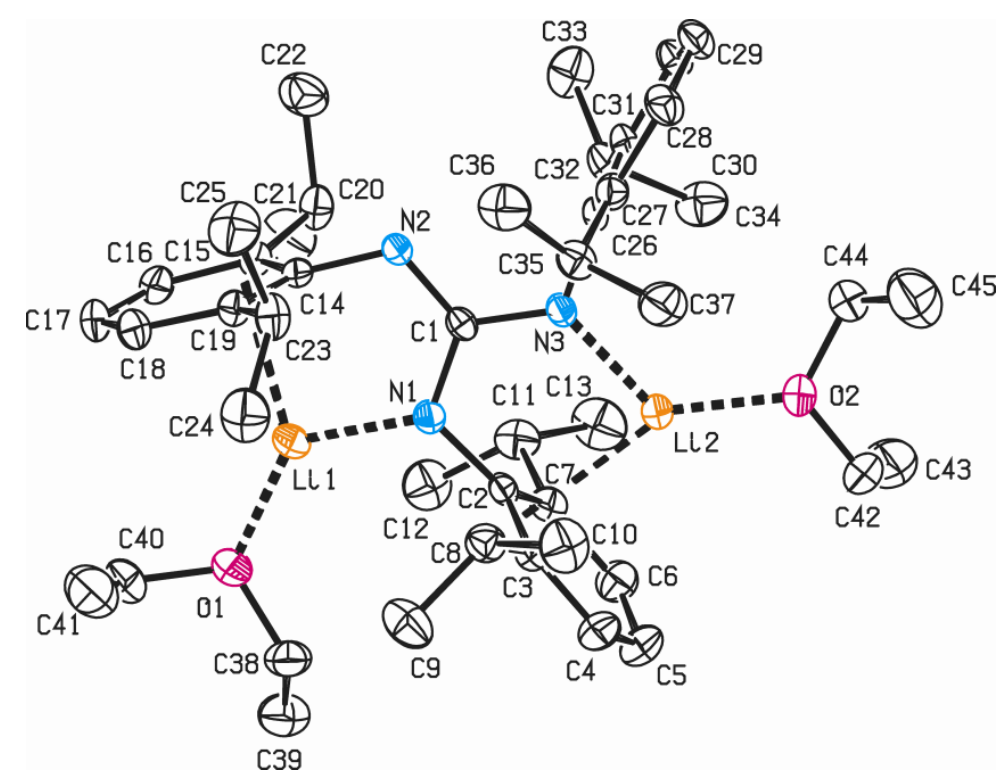

Figure S6 Molecular structure of $\mathbf{5}$ (ORTEP view, 50\% probability level). Hydrogen atoms and the second independent molecule are omitted for clarity. Selected interatomic distances $[\AA \AA]$ and angles $\left[{ }^{\circ}\right]$, values of the second independent molecule are given in italics in parentheses: Li1-N1 1.907(5) (1.917(5)), Li1-Cg(C15, C14, C19) 2.275(4) (3.137(5)), Li1-O1 1.938(5) (1.940(5)), Li2-N3 1.892(5) (1.893(5)), Li2-Cg(C3, C2, C7) 2.300(4) (2.304(6)), Li2-O2 1.925(5) (1.913(5)), C1-N1 1.378(3) (1.383(3)), C1-N2 1.338(3) (1.330(3)), C1-N3 1.361(3) (1.359(3)); N1-Li1-Cg(C15, C14, C19) 87.63(5) (86.33(6)), N1-Li1-O1 126.5(3) (125.4(3)), N3-Li2-Cg(C3, C2, C7) 87.65(6) (84.66(5)), N3-Li2-O2 125.5(3) (125.9(3)), N1-C1-N2 120.8(2) (120.8(2)), N1-C1-N3 118.1(2) (117.9(2)), N2-C1-N3 121.0(2) $(121.3(2))$.
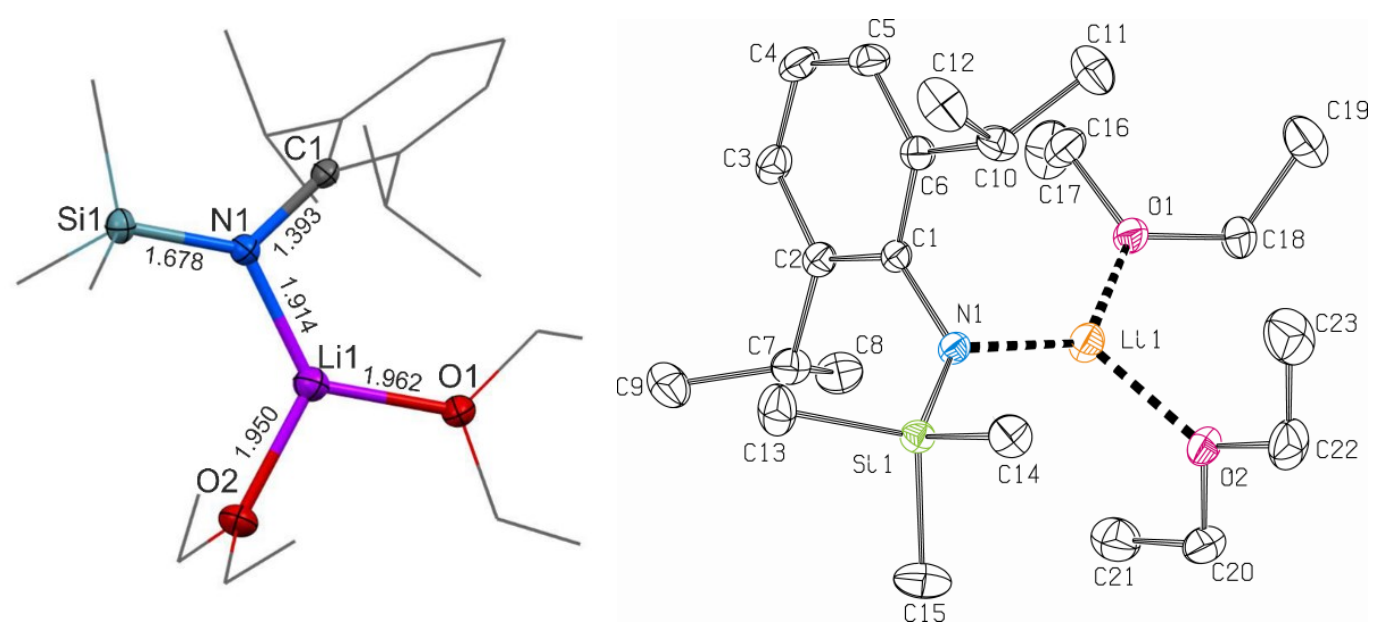

Figure S7 Molecular structure of 6. $\left[\mathrm{Et}_{2} \mathrm{O}\right]_{2}$ (schematic and ORTEP view, $40 \%$ probability level). Hydrogen atoms are omitted for clarity. Selected interatomic distances [Å] and angles [ $\left.{ }^{\circ}\right]$ : Li1-N1 1.914(3), Li1-O1 1.962(3), Li1-O2 1.950(3); N1-Li1-O1 128.08(14), N1-Li1-O2 123.53(14). 

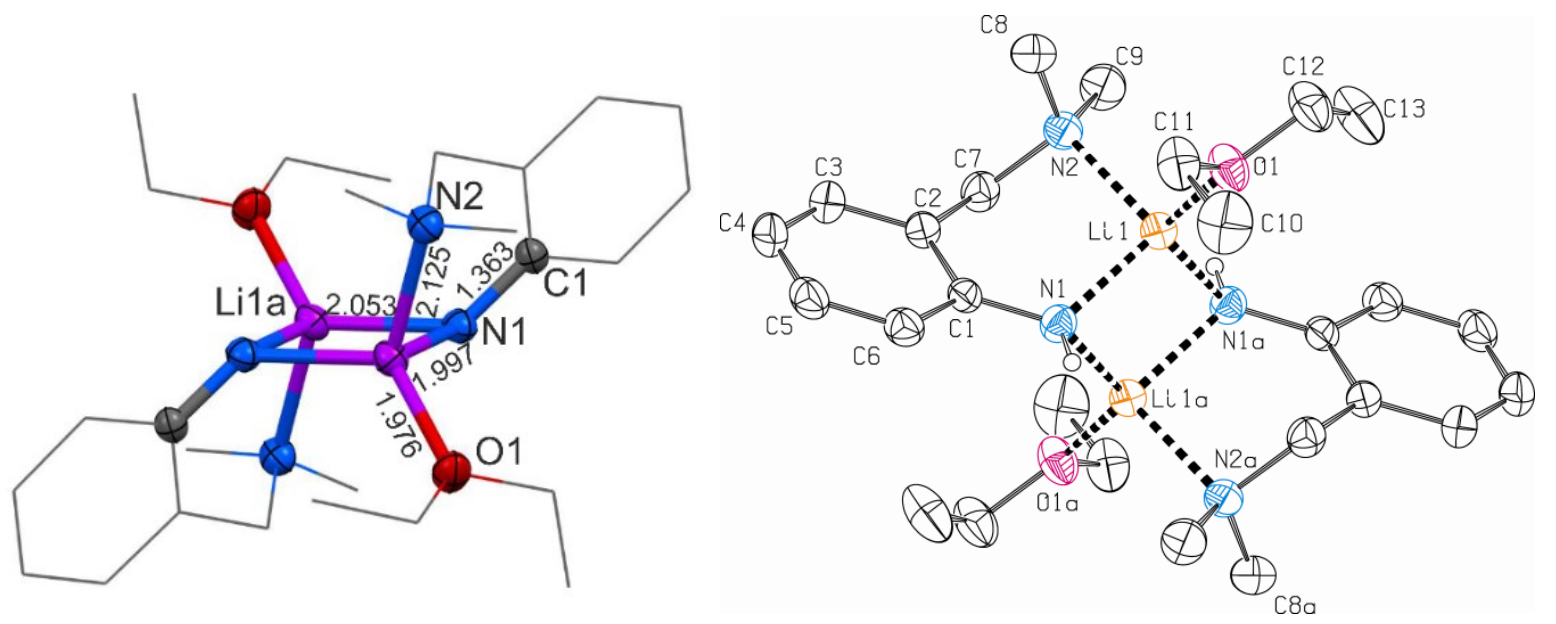

Figure S8 Molecular structure of $\left[7 . \mathrm{Et}_{2} \mathrm{O}\right]_{2}$ (schematic and ORTEP view, 30\% probability level). Hydrogen atoms are omitted for clarity. Selected interatomic distances [Å] and angles [ $\left.{ }^{\circ}\right]$ : Li1-N1 1.996(5), Li1-N1a 2.053(5), Li1-N2 2.125(5), Li1-O1 1.975(5); N1-Li1-N2 96.9(2), N1-Li1-N1a 103.6(2), N2-Li1-N1a 108.0(2).

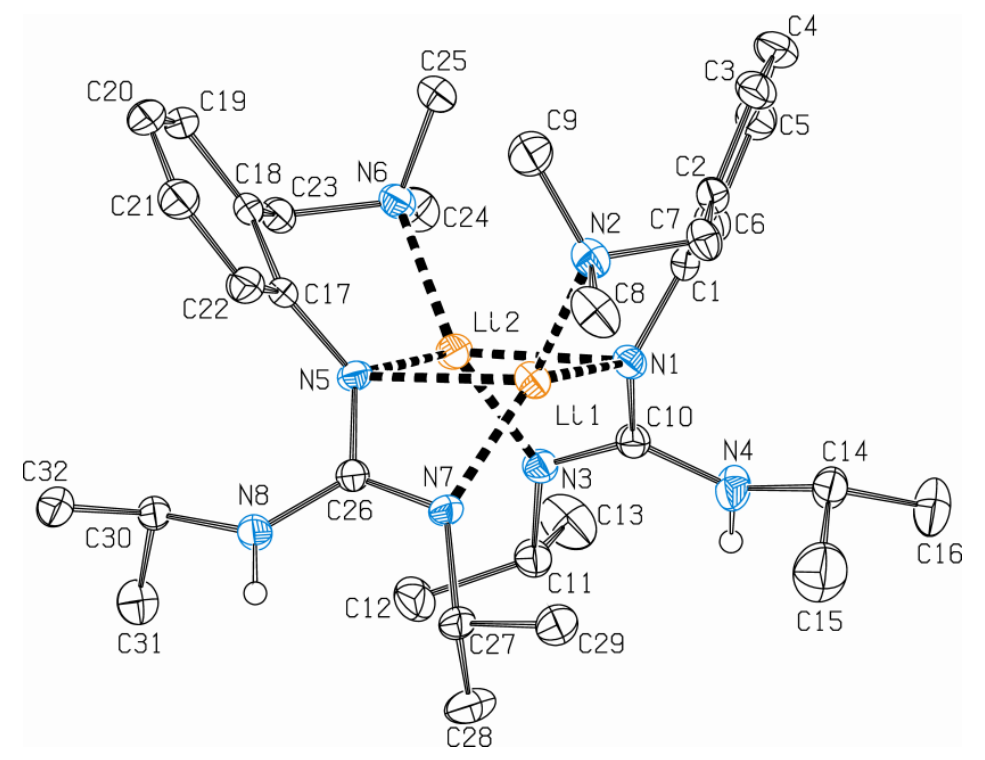

Figure S9 Molecular structure of $\mathbf{8}$ (ORTEP view, 40\% probability level). Hydrogen atoms are omitted for clarity. Selected interatomic distances [Å] and angles [ ${ }^{\circ}$ ]: Li1-Li2 2.456(4), Li1-N1 1.978(3), Li1-N5 2.260(3), Li1-N2 2.027(3), Li1-N7 1.948(3), Li2-N1 2.223(3), Li2-N5 1.965(3), Li2-N6 2.057(3), Li2-N3 1.964(3), C10-N1 1.393(2), C10-N3 1.296(2), C10 N4 1.369(2), C26-N5 1.3879(19), C26-N7 1.301(2), C26-N8 1.369(2); N7-Li1-N5 65.55(10), Li1-N1-Li2 71.27(12), N1-Li2-N5 109.96(13), Li2-N5-Li1 70.67(12), N1-C10-N3 117.31(14), N5-C26-N7 116.98(14). 


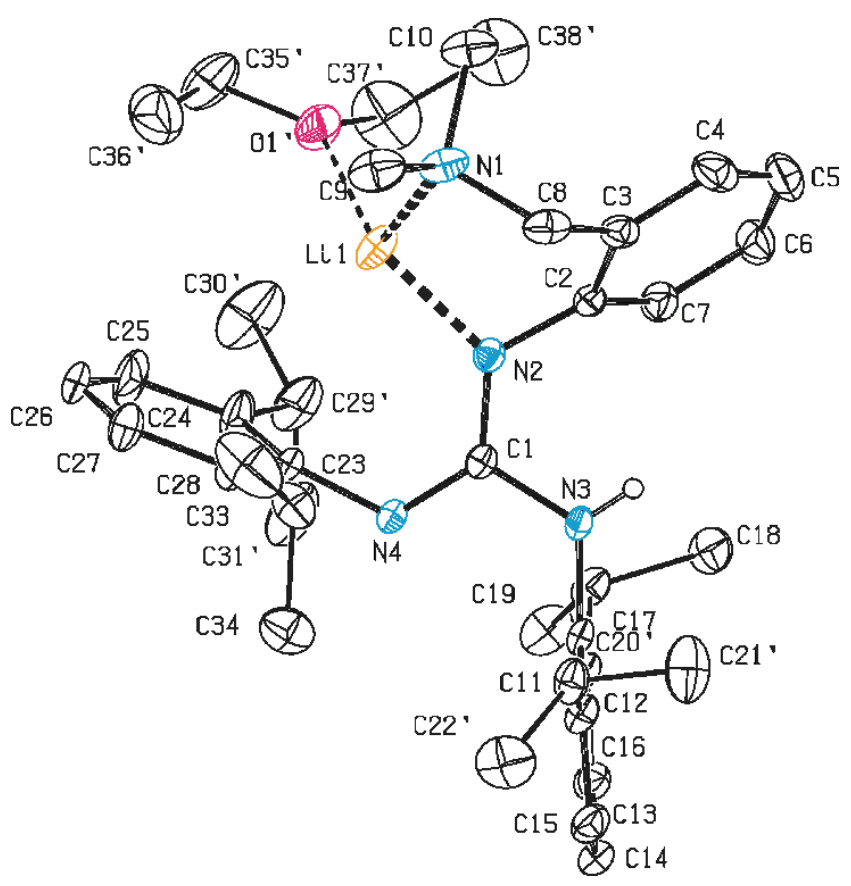

Figure S10 Molecular structure of $\mathbf{9}$ (ORTEP view, 30\% probability level). Disorder of the aromatic ring, free molecule of diethylether, four atoms from diethylether bonding and hydrogen atoms are omitted for clarity. Selected interatomic distances [Å] and angles [ ${ }^{\circ}$ ]: Li1-N1 2.109(8), Li1-N2 1.939(7), Li1-N4 3.431(10), Li1-O1' 1.987(11), Li1-Cg(C24A, C23A, C28A) 3.042(5), C1-N2 1.350(5), C1-N3 1.412(4), C1-N4 1.299(5); N1-Li1-N2 101.5(3), N1-Li1-O1' 103.8(5), N2-Li1-O1' 130.6(5), N2-C1-N4 126.5(3).

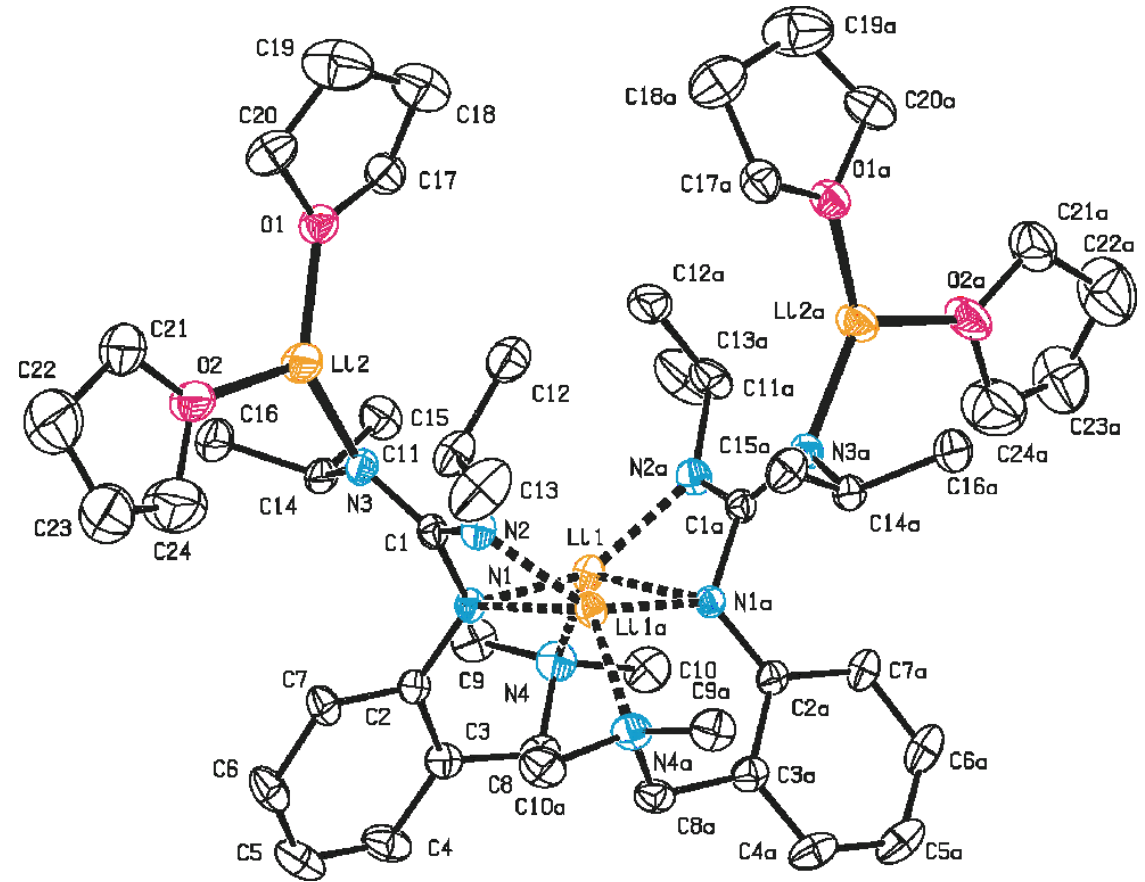

Figure S11 Molecular structure of $\mathbf{1 0}$ (ORTEP view, 40\% probability level). Hydrogen atoms are omitted for clarity. Selected interatomic distances [ $\AA \AA$ ] and angles [ $\left.{ }^{\circ}\right]$ : Li1-N1 1.968(9), Li1-N1a 
2.251(8), Li1-N4 2.041(9), Li1a-N2 1.941(9), Li2-N3 1.928(10), Li2-O1 1.903(11), Li2-O2 1.949(11), C1N1 1.433(6), C1-N2 1.323(6), C1-N3 1.352(6); N1-Li1-N4 96.5(4), N1-Li1-N1a 102.4(3), N4-Li1-N1a 124.8(4), Li1-N1-C1 117.7(4), N3-Li2-O1 129.0(5), N3-Li2-O2 116.0(5), N1-C1-N2 112.9(4), N1-C1-N3 122.2(4), N2-C1-N3 124.8(4), Li1-N1-Li1a 76.9(3).

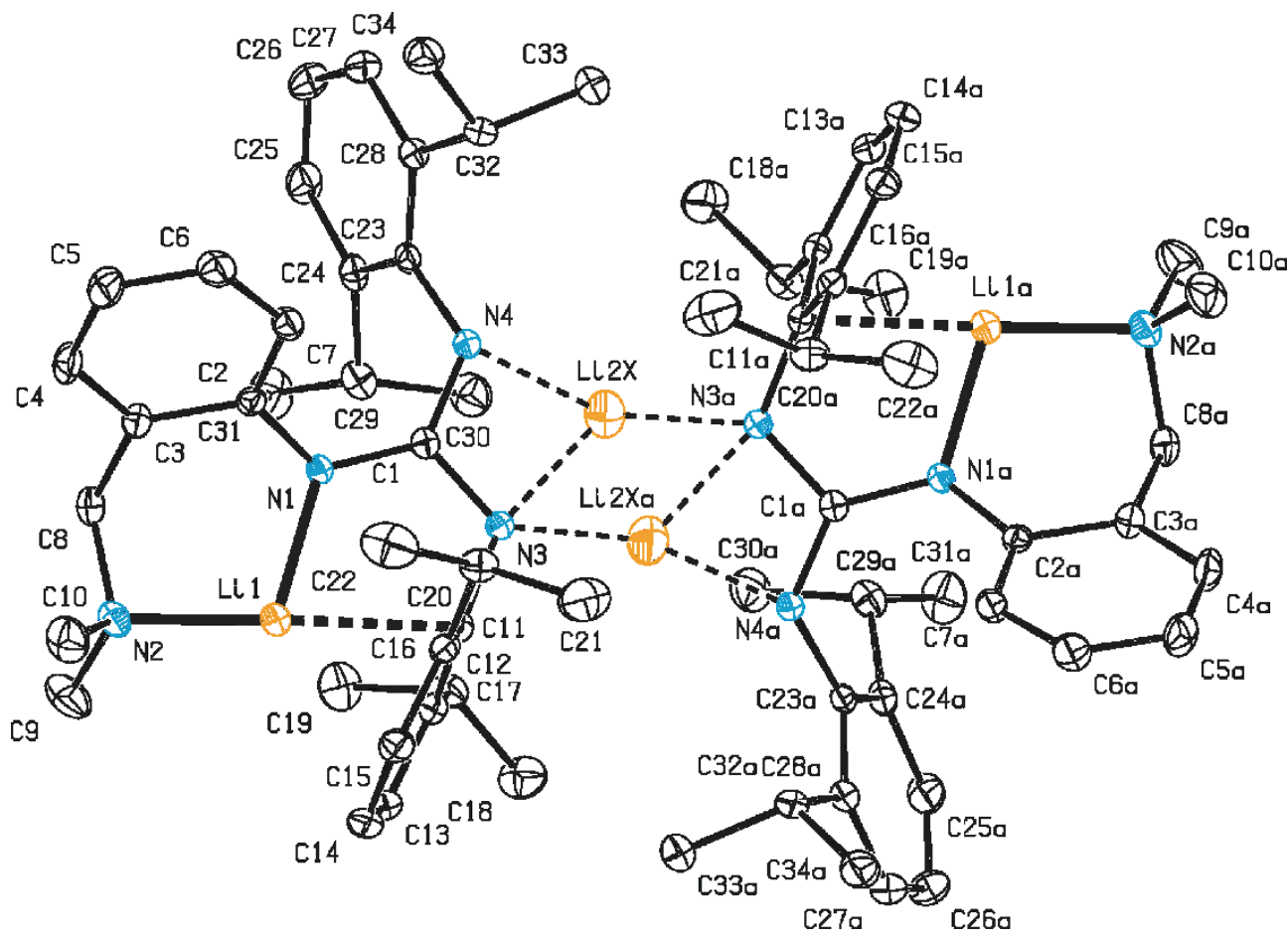

Figure S12 Molecular structure of 11 (ORTEP view, 40\% probability level). Disordered part and hydrogen atoms are omitted for clarity. Selected interatomic distances [Å] and angles [ $\left.{ }^{\circ}\right]$ : Li1-N1 1.940(4), Li1-N2 1.982(4), Li1-Cg1 (C11, C12, C13, C14, C15, C16) 2.173(4), Li1-Cg2 (C15, C16, C11, C12, C13) 2.130(4), C1-N1 1.366(3), C1-N3 1.369(3), C1-N4 1.350(2); Li1X-N3 2.167(15), Li1Xa-N3a 2.011(15), Li1Xa-N4 1.875(15), Li2X-N3 1.993(11), Li2X-N3a 1.920(10), Li2X-N4 1.955(10); N1-Li1-N2 105.85(19), N1-C1-N3 118.24(17), N3-C1-N4 113.81(17), N3-Li1Xa-N3a 110.1(8), N3-Li1Xa-N4a 145.7(11), N3a-Li1Xa-N4a 68.2(5), N3-Li2X-N4 70.5(3), N3-Li2X-N3a 122.2(6), N4-Li2X-N3a 146.8(7). 


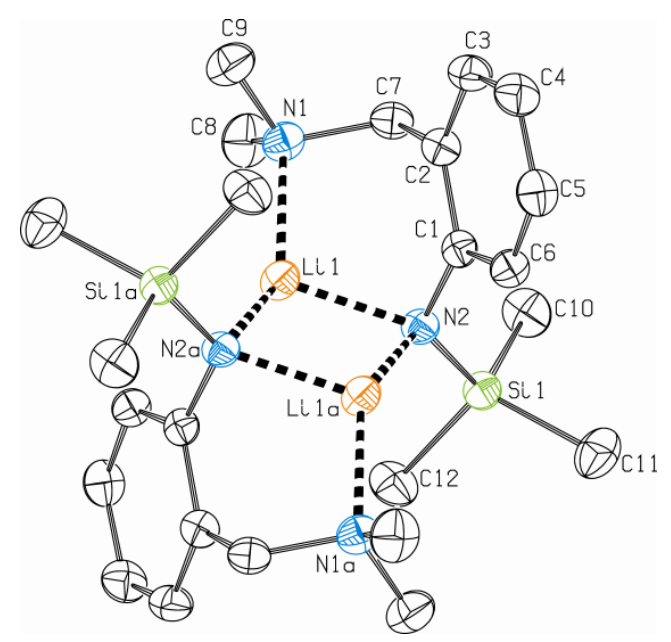

Figure S13 Molecular structure of $[12]_{2}$ (ORTEP view, 50\% probability level). Hydrogen atoms are omitted for clarity. Selected interatomic distances [Å] and angles [ ${ }^{\circ}$ ]: Li1-N1 2.049(4), Li1-N2 1.984(4), Li1-N2a 2.019(4); N1-Li1-N2 105.24(17), N1-Li1-N2a 139.31(19), Li1-N2-Li1a 76.47(16).

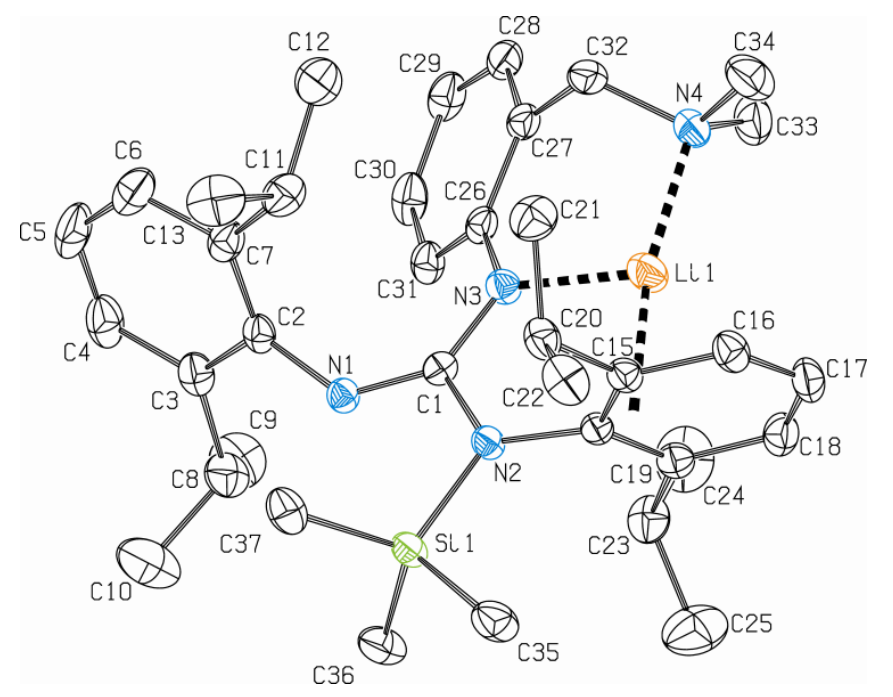

Figure S14 Molecular structure of 14 (ORTEP view, 50\% probability level). Hydrogen atoms are omitted for clarity. Selected interatomic distances [Å] and angles [ $\left.{ }^{\circ}\right]$ : Li1-N3 1.935(4), Li1-N4 1.986(4), Li1-N2 3.015(4), Li1-Cg(C15, C14, C19) 2.151(4), C1-N1 1.303(2), C1-N2 1.415(2), C1-N3 1.359(2); N3Li1-N4 105.67(17), Li1-N3-C1 122.93(15), N2-C1-N3 114.48(15). 


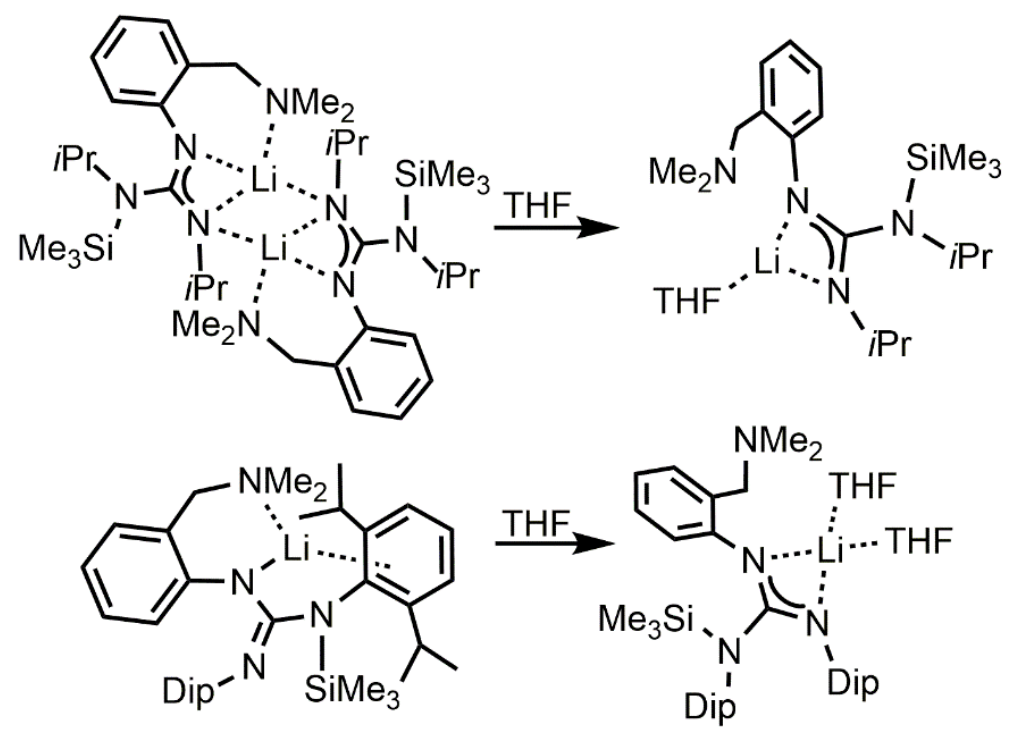

Scheme S1 Proposed THF solvation of 13 (above) and 14 (below).

\section{General methods}

All syntheses were performed using the standard Schlenk techniques under an inert argon atmosphere. All solvents and starting carbodiimides were purchased from commercial sources (Sigma-Aldrich and VúOS, a. s. from Pardubice-Rybitví). Solvents were dried with the help of solvent purification system PureSolv MD 7 supplied by Innovative Technology, Inc., degassed and then stored under argon atmosphere. Single crystals suitable for $\mathrm{X}$-ray analyses were obtained under argon from corresponding saturated solutions of products in organic solvent(s) cooled to 7 or $-30^{\circ} \mathrm{C}$.

Melting points were measured in an inert perfluoroalkylether and were uncorrected. Deuterated solvents for NMR spectra were distilled, degassed and stored over a $\mathrm{K}$ or Na-mirror under argon atmosphere.

NMR spectra in THF- $d_{8}$ and/or in $\mathrm{C}_{6} \mathrm{D}_{6}$ of starting lithium amides $\mathbf{1}, \mathbf{6}, \mathbf{7}$ and 12 were measured for comparison and studies of chemical shifts of target compounds. Synthesis of $\mathbf{1}^{1}, 6^{2}, \mathbf{7}^{3}$ and $12^{3}$ is reported elsewhere. NMR spectra were recorded from solutions of appropriate compounds in deuterated solvent(s) on a Bruker Avance 500 spectrometer (equipped with Z-gradient $5 \mathrm{~mm}$ probe) at frequencies for ${ }^{1} \mathrm{H}(500.13 \mathrm{MHz}),{ }^{13} \mathrm{C}\left\{{ }^{1} \mathrm{H}\right\}(125.76 \mathrm{MHz}),{ }^{7} \mathrm{Li}\left\{{ }^{1} \mathrm{H}\right\}(194.37 \mathrm{MHz})$ at $295 \mathrm{~K}$. Solutions were obtained by dissolving of approximately $40 \mathrm{mg}$ of each compound approximately in $0.6 \mathrm{ml}$ of deuterated solvents. Values of ${ }^{1} \mathrm{H}$ chemical shifts were calibrated to internal standard tetramethylsilane $\left(\delta\left({ }^{1} \mathrm{H}\right)=0.00\right)$ or to residual signals of benzene $\left(\delta\left({ }^{1} \mathrm{H}\right)=7.16\right)$ and $\operatorname{THF}\left(\delta\left({ }^{1} \mathrm{H}\right)=3.58\right.$ or 1.73), respectively. Values of ${ }^{13} \mathrm{C}$ chemical shifts were calibrated to signals of THF $\left(\delta\left({ }^{13} \mathrm{C}\right)=67.6\right)$ or benzene $\left(\delta\left({ }^{13} \mathrm{C}\right)=128.4\right)$ and ${ }^{7} \mathrm{Li}$ to external $1 \mathrm{M} \mathrm{LiCl}$ in $\mathrm{D}_{2} \mathrm{O}\left(\delta\left({ }^{7} \mathrm{Li}\right)=0.0\right.$; recalculated to frequency ratio $\Xi$ for $500.13 \mathrm{MHz}$ spectrometer - 194.3695079361 MHz). All ${ }^{13} \mathrm{C}$ NMR spectra were measured 
using standard proton-decoupled experiment and $\mathrm{CH}$ and $\mathrm{CH}_{3}$ vs. $\mathrm{C}$ and $\mathrm{CH}_{2}$ were differentiated by the help of APT method ${ }^{4}$. NMR spectra in a scanned forms ${ }^{*}$ of printed versions of prepared compounds 2 - 14 (expect of 10) are attached at the end of this document.

The X-ray data for colorless crystals of $2-5,6 .\left[\mathrm{Et}_{2} \mathrm{O}\right]_{2},\left[7 . \mathrm{Et}_{2} \mathrm{O}\right]_{2}, 8-11,[12]_{2}$ and 14 (see Table S1) were obtained at $150 \mathrm{~K}$ using Oxford Cryostream low-temperature device on a Nonius KappaCCD diffractometer with $\mathrm{Mo} \mathrm{K}_{\alpha}$ radiation $(\lambda=0.71073 \AA)$, a graphite monochromator, and the $\phi$ and $\chi$ scan mode. Data reductions were performed with DENZO-SMN ${ }^{5}$. The absorption was corrected by integration methods. ${ }^{6}$ Structures were solved by direct methods $(\text { Sir92 })^{7}$ and refined by full matrix least-square based on $F^{2}$ (SHELXL97) ${ }^{8}$. Hydrogen atoms were mostly localized on a difference Fourier map, however to ensure uniformity of treatment of crystal, all hydrogen were recalculated into idealized positions (riding model) and assigned temperature factors $\mathrm{H}_{\text {iso }}(\mathrm{H})=1.2 \mathrm{U}_{\text {eq }}$ (pivot atom) or of $1.5 \mathrm{U}_{\text {eq }}$ (methyl). $\mathrm{H}$ atoms in methyl, methylene, methane moieties and hydrogen atoms in aromatic rings were placed with $\mathrm{C}-\mathrm{H}$ distances of $0.96,0.97,0.98$ and $0.93 \AA$ and $0.86 \AA$ for $\mathrm{N}-\mathrm{H}$ bonds.

Crystallographic data for structural analysis have been deposited with the Cambridge Crystallographic Data Centre, CCDC no. $1997777-1997791$ for 2 - 5, 6. $\left[\mathrm{Et}_{2} \mathrm{O}\right]_{2},\left[\right.$ 7. $\mathrm{Et}_{2} \mathrm{O}_{2}, 8$ - 11, [12] 2 and 14 and its solvatopolymorphs, respectively. Copies of this information may be obtained free of charge from The Director, CCDC, 12 Union Road, Cambridge CB2 1EY, UK (fax: +44-1223-336033; email: deposit@ccdc.cam.ac.uk or www: http://www.ccdc.cam.ac.uk).

* Unfortunately, all the spectral data in the digital form were lost during the crash of the hard-disc, so we have only printed versions of it. We apologize that most of the spectra are in the working versions and containing some indelible marks/symbols. In some cases, there are also the dirty lines caused by cartridge of the printer present on the sheets. 
Table S1 Crystallographic data for $2-5,6 .\left[\mathrm{Et}_{2} \mathrm{O}\right]_{2},\left[7 . \mathrm{Et}_{2} \mathrm{O}\right]_{2}, 8-11,[12]_{2}$ and 14.

\begin{tabular}{|c|c|c|c|c|c|c|c|}
\hline Compound reference & 2 & $2 a$ & 3 & 4 & $4 a$ & 5 & 6. $\left[\mathrm{Et}_{2} \mathrm{O}\right]_{2}$ \\
\hline Chemical formula & $\mathrm{C}_{42} \mathrm{H}_{74} \mathrm{Li}_{2} \mathrm{~N}_{6} \mathrm{O}$ & $\mathrm{C}_{46} \mathrm{H}_{80} \mathrm{Li}_{2} \mathrm{~N}_{6} \mathrm{O}_{2}$ & $\mathrm{C}_{41} \mathrm{H}_{62} \mathrm{LiN}_{3} \mathrm{O}$ & $\mathrm{C}_{50} \mathrm{H}_{86} \mathrm{Li}_{4} \mathrm{~N}_{6} \mathrm{O}_{3}$ & $\mathrm{C}_{46} \mathrm{H}_{82} \mathrm{Li}_{4} \mathrm{~N}_{6} \mathrm{O}_{2}$ & $\mathrm{C}_{45} \mathrm{H}_{71} \mathrm{Li}_{2} \mathrm{~N}_{3} \mathrm{O}_{2}$ & $\mathrm{C}_{23} \mathrm{H}_{46} \mathrm{LiNO}_{2} \mathrm{Si}$ \\
\hline Formula Mass & 692.95 & 763.04 & 619.88 & 847.00 & 778.93 & 699.93 & 403.64 \\
\hline Crystal system & Orthorhombic & Orthorhombic & Monoclinic & Monoclinic & Monoclinic & Monoclinic & Monoclinic \\
\hline$a / \AA$ & $22.727(3)$ & $16.3369(6)$ & $11.3080(10)$ & $14.7360(13)$ & $18.557(2)$ & $10.8440(4)$ & $9.5490(9)$ \\
\hline$b / \AA$ & $17.001(2)$ & $16.3842(4)$ & $17.4330(18)$ & $18.5181(19)$ & $10.1912(8)$ & $20.7690(15)$ & $16.4230(12)$ \\
\hline$c / \AA$ & $23.279(3)$ & $17.0330(3)$ & $21.871(2)$ & $21.7870(14)$ & $27.210(2)$ & $19.532(2)$ & $17.6211(18)$ \\
\hline$\alpha /^{\circ}$ & 90 & 90 & 90 & 90 & 90 & 90 & 90 \\
\hline$B /^{\circ}$ & 90 & 90 & $115.010(8)$ & $116.988(5)$ & $106.825(4)$ & $94.604(5)$ & 103.391(7) \\
\hline$\gamma /{ }^{\circ}$ & 90 & 90 & 90 & 90 & 90 & 90 & 90 \\
\hline Unit cell volume $/ \AA^{3}$ & $8995.3(2)$ & $4559.2(2)$ & $3907.2(7)$ & $5297.8(8)$ & $4925.6(8)$ & $4384.8(6)$ & $2688.3(4)$ \\
\hline Temperature/K & $150(1)$ & $150(1)$ & $150(1)$ & $150(1)$ & $150(1)$ & $150(1)$ & $150(1)$ \\
\hline Space group & Pbca & Pbca & $P 2_{1} / c$ & $P 2_{1} / c$ & $P 2_{1} / c$ & $P 2_{1} / c$ & $P 2_{1} / c$ \\
\hline $\begin{array}{l}\text { No. of formula units per unit } \\
\text { cell, } Z\end{array}$ & 8 & 4 & 4 & 4 & 4 & 4 & 4 \\
\hline Absorption coefficient, $\mu / \mathrm{mm}^{-1}$ & 0.061 & 0.061 & 0.062 & 0.064 & 0.063 & 0.063 & 0.103 \\
\hline No. of reflections measured & 34953 & 35534 & 23182 & 64327 & 49374 & 40168 & 23741 \\
\hline No. of independent reflections & 8592 & 5132 & 8678 & 11421 & 8269 & 17026 & 5907 \\
\hline$R_{\text {int }}$ & 0.0402 & 0.0202 & 0.0462 & 0.1211 & 0.0605 & 0.0393 & 0.0275 \\
\hline Final $R_{1}$ values $(I>2 \sigma(I))$ & 0.0569 & 0.0419 & 0.0711 & 0.0619 & 0.1384 & 0.0556 & 0.0410 \\
\hline Final $w R\left(F^{2}\right)$ values $(I>2 \sigma(I))$ & 0.1152 & 0.0964 & 0.1516 & 0.1308 & 0.2714 & 0.1163 & 0.0901 \\
\hline Goodness of fit on $F^{2}(S)$ & 1.180 & 1.119 & 1.135 & 1.131 & 1.084 & 1.222 & 1.149 \\
\hline
\end{tabular}


Table S1 Crystallographic data for 2 - 5, 6. $\left[\mathrm{Et}_{2} \mathrm{O}\right]_{2},\left[7 . \mathrm{Et}_{2} \mathrm{O}\right]_{2}, 8-11,[12]_{2}$ and 14 - continuing.

\begin{tabular}{|c|c|c|c|c|c|c|c|}
\hline Compound reference & {$\left[\text { 7. } \mathrm{Et}_{2} \mathrm{O}\right]_{2}$} & 8 & 9 & 10 & 11 & {$[12]_{2}$} & 14 \\
\hline Chemical formula & $\mathrm{C}_{26} \mathrm{H}_{46} \mathrm{Li}_{2} \mathrm{~N}_{4} \mathrm{O}_{2}$ & $\mathrm{C}_{32} \mathrm{H}_{54} \mathrm{Li}_{2} \mathrm{~N}_{8}$ & $\mathrm{C}_{40} \mathrm{H}_{62} \mathrm{LiN}_{4} \mathrm{O}_{1.5}$ & $\mathrm{C}_{48} \mathrm{H}_{84} \mathrm{Li}_{4} \mathrm{~N}_{8} \mathrm{O}_{4}$ & $\mathrm{C}_{68} \mathrm{H}_{92} \mathrm{Li}_{4} \mathrm{~N}_{8}$ & $\mathrm{C}_{24} \mathrm{H}_{42} \mathrm{Li}_{2} \mathrm{~N}_{4} \mathrm{Si}_{2}$ & $\mathrm{C}_{37} \mathrm{H}_{55} \mathrm{LiN}_{4} \mathrm{Si}$ \\
\hline Formula Mass & 460.55 & 564.71 & 629.88 & 864.99 & 1049.26 & 456.68 & 590.88 \\
\hline Crystal system & Orthorhombic & Triclinic & Monoclinic & Tetragonal & Monoclinic & Monoclinic & Monoclinic \\
\hline$a / \AA ̊$ & $11.0950(8)$ & $9.7201(5)$ & $10.9840(11)$ & $13.2530(14)$ & $20.8732(5)$ & $9.4890(9)$ & $12.2490(12)$ \\
\hline$b / \AA ̊$ & $13.6730(9)$ & $9.9280(6)$ & $19.1401(16)$ & $13.2530(14)$ & $16.8446(8)$ & $17.8541(10)$ & $16.0520(6)$ \\
\hline$c / \AA ̊$ & $18.9611(15)$ & 19.3199(11) & $19.0120(17)$ & $29.704(3)$ & $18.2813(6)$ & $9.2600(8)$ & $20.7821(13)$ \\
\hline$\alpha /^{\circ}$ & 90 & $94.410(5)$ & 90 & 90 & 90 & 90 & 90 \\
\hline$B /{ }^{\circ}$ & 90 & $101.834(5)$ & $105.607(8)$ & 90 & $100.782(4)$ & $116.176(5)$ & $118.318(8)$ \\
\hline$v /^{\circ}$ & 90 & $105.387(4)$ & 90 & 90 & 90 & 90 & 90 \\
\hline Unit cell volume $/ \AA^{3}$ & $2876.4(4)$ & $1742.24(18)$ & $3849.6(6)$ & $5217.3(12)$ & $6314.2(4)$ & $1407.9(2)$ & $3597.2(5)$ \\
\hline Temperature/K & $150(1)$ & $150(1)$ & $150(1)$ & $150(1)$ & $150(1)$ & $150(1)$ & $150(1)$ \\
\hline Space group & Pbcn & $P 2_{1} / c$ & $P 2_{1} / c$ & $P 4_{1} 2{ }_{1} 2$ & $C 2 / c$ & $P 2_{1} / c$ & $P 2_{1} / c$ \\
\hline No. of formula units per unit cell, $Z$ & 4 & 2 & 4 & 4 & 4 & 2 & 4 \\
\hline Absorption coefficient, $\mu / \mathrm{mm}^{-1}$ & 0.066 & 0.065 & 0.065 & 0.069 & 0.064 & 0.143 & 0.095 \\
\hline No. of reflections measured & 14239 & 34712 & 28082 & 19509 & 25260 & 11452 & 22808 \\
\hline No. of independent reflections & 2684 & 7944 & 7337 & 4465 & 7016 & 3179 & 7724 \\
\hline$R_{\text {int }}$ & 0.0773 & 0.0389 & 0.0789 & 0.0389 & 0.0440 & 0.0484 & 0.0344 \\
\hline Final $R_{1}$ values $(I>2 \sigma(I))$ & 0.0730 & 0.0546 & 0.1151 & 0.0762 & 0.0614 & 0.0554 & 0.0489 \\
\hline Final $w R\left(F^{2}\right)$ values $(I>2 \sigma(I))$ & 0.1531 & 0.1116 & 0.2309 & 0.1778 & 0.1319 & 0.1232 & 0.0971 \\
\hline Goodness of fit on $F^{2}(S)$ & 1.014 & 1.131 & 1.126 & 1.151 & 1.161 & 1.147 & 1.160 \\
\hline
\end{tabular}




\section{Lithium [2,6-di(propan-2-yl)phenyl]azanide (1)}

${ }^{1} \mathrm{H}$ NMR (THF-d 8,500 MHz, 295 K) $\delta: 6.68$ (br s, 2H, ArH); 6.05 (br s, 1H, ArH); 3.19 (br s, 2H, CH); 2.70 (br s, $1 \mathrm{H}, \mathrm{NH}$ ); 1.19 (d, ${ }^{2} \mathrm{~J}=6.8 \mathrm{~Hz}, 12 \mathrm{H}, \mathrm{CH}_{3}$ ). ${ }^{7} \mathrm{Li}$ NMR (THF-d $8,194 \mathrm{MHz}, 295 \mathrm{~K}$ ) $\delta:-0.7 . \ln { }^{13} \mathrm{C} \mathrm{NMR}$ spectrum in THF- $\mathrm{d}_{8}(125 \mathrm{MHz}, 295 \mathrm{~K})$ only the very broad signals were observed because of fast exchange processes.

\section{Lithium trimethylsilyl[2,6-di(propan-2-yl)phenyl]azanide (6)}

${ }^{1} \mathrm{H}$ NMR $\left(\mathrm{C}_{6} \mathrm{D}_{6}, 500 \mathrm{MHz}, 295 \mathrm{~K}\right) \delta: 7.00\left(\mathrm{~d},{ }^{3} \mathrm{~J}=7.6 \mathrm{~Hz}, 2 \mathrm{H}, \operatorname{ArH}\right) ; 6.87\left(\mathrm{t},{ }^{3} \mathrm{~J}=7.6 \mathrm{~Hz}, 1 \mathrm{H}, \mathrm{ArH}\right) ; 3.49(\mathrm{~m}$, $2 \mathrm{H}, \mathrm{CH}) ; 1.20\left(\mathrm{~d},{ }^{3} \mathrm{~J}=6.9 \mathrm{~Hz}, 12 \mathrm{H}, \mathrm{CH}_{3}\right) ; 0.12\left(\mathrm{~s}, 9 \mathrm{H}, \mathrm{Si}\left(\mathrm{CH}_{3}\right)_{3}\right) .{ }^{7} \mathrm{Li} \mathrm{NMR}\left(\mathrm{C}_{6} \mathrm{D}_{6}, 194 \mathrm{MHz}, 295 \mathrm{~K}\right) \delta: 1.1 .{ }^{1} \mathrm{H}$ NMR (THF-d 8,500 MHz, 295 K) 8: 6.72 (br s, 2H, ArH); 6.33 (br s, 1H, ArH); 4.06 (br s, 2H, CH); 1.06 (br

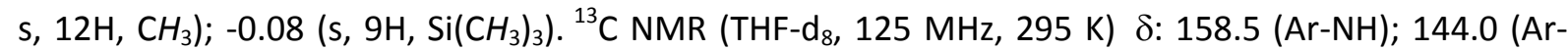
$\mathrm{CH}) ; 122.1(\mathrm{Ar}) ; 115.0(\mathrm{Ar}) ; 26.8(\mathrm{CH}) ; 25.5\left(\mathrm{CH}_{3}\right) ; 4.6\left(\mathrm{Si}\left(\mathrm{CH}_{3}\right)_{3}\right)$. ${ }^{7} \mathrm{Li}$ NMR (THF-d $\left.8,194 \mathrm{MHz}, 295 \mathrm{~K}\right) \delta$ : 0.3 .

\section{Lithium 2-[(dimethylamino)methyl]phenylazanide (7)}

${ }^{1} \mathrm{H}$ NMR $\left(\mathrm{C}_{6} \mathrm{D}_{6}, 500 \mathrm{MHz}, 295 \mathrm{~K}\right) \delta: 7.13\left(\mathrm{t},{ }^{3} \mathrm{~J}=7.2 \mathrm{~Hz}, 1 \mathrm{H}, \operatorname{ArH}\right) ; 6.79\left(\mathrm{~d},{ }^{3} \mathrm{~J}=6.8 \mathrm{~Hz}, 1 \mathrm{H}, \operatorname{ArH}\right) ; 6.60(\mathrm{~d}$, $\left.{ }^{3} \mathrm{~J}=7.1 \mathrm{~Hz}, 1 \mathrm{H}, \mathrm{ArH}\right) ; 6.48\left(\mathrm{t},{ }^{3} \mathrm{~J}=7.2 \mathrm{~Hz}, 1 \mathrm{H}, \mathrm{ArH}\right) ; 2.97\left(\mathrm{br} \mathrm{s}, 2 \mathrm{H}, \mathrm{CH}_{2}\right) ; 2.73(\mathrm{~s}, 1 \mathrm{H}, \mathrm{NH}) ; 1.70(\mathrm{~s}, 6 \mathrm{H}$, $\left.\left(\mathrm{CH}_{3}\right)_{2}\right) .{ }^{7} \mathrm{LiNMR}\left(\mathrm{C}_{6} \mathrm{D}_{6}, 194 \mathrm{MHz}, 295 \mathrm{~K}\right) \delta: 1.8 .{ }^{1} \mathrm{H}$ NMR (THF-d $\left.8,500 \mathrm{MHz}, 295 \mathrm{~K}\right) \delta: 6.61(\mathrm{~m}, 2 \mathrm{H}, \mathrm{ArH})$; $6.20(\mathrm{~s}, 1 \mathrm{H}, \mathrm{ArH}) ; 5.82(\mathrm{~s}, 1 \mathrm{H}, \mathrm{ArH}) ; 3.25\left(\mathrm{br} \mathrm{s}, 2 \mathrm{H}, \mathrm{CH}_{2}\right) ; 2.82(\mathrm{~s}, 1 \mathrm{H}, \mathrm{NH}) ; 2.10\left(\mathrm{~s}, 6 \mathrm{H},\left(\mathrm{CH}_{3}\right)_{2}\right) .{ }^{13} \mathrm{C} \mathrm{NMR}$ (THF-d $\left.\mathrm{d}_{8}, 125 \mathrm{MHz}, 295 \mathrm{~K}\right)$ 8: $164.2\left(\mathrm{Ar}-\mathrm{NH}_{2}\right) ; 132.3$ (Ar); 129.4 (Ar); $120.4\left(\mathrm{Ar}-\mathrm{CH}_{2}\right) ; 118.6$ (Ar); 108.1 (Ar); $65.8\left(\mathrm{CH}_{2}\right) ; 45.4\left(\mathrm{CH}_{3}\right) .{ }^{7} \mathrm{Li}$ NMR (THF-d 8 , $\left.194 \mathrm{MHz}, 295 \mathrm{~K}\right) \delta: 1.3$.

\section{Lithium trimethylsilyl-2-[(dimethylamino)methyl]phenylazanide (12)}

${ }^{1} \mathrm{H}$ NMR $\left(\mathrm{C}_{6} \mathrm{D}_{6}, 500 \mathrm{MHz}, 295 \mathrm{~K}\right) \delta: 7.02(\mathrm{~s}, 1 \mathrm{H}, \operatorname{ArH}) ; 6.89(\mathrm{~s}, 1 \mathrm{H}, \operatorname{ArH}) ; 6.66(\mathrm{~s}, 1 \mathrm{H}, \operatorname{ArH}) ; 6.53(\mathrm{~s}, 1 \mathrm{H}$, $\mathrm{ArH}) ; 3.89,2.51\left(\mathrm{br} \mathrm{s}, \mathrm{AX}\right.$ spin system, 2H, diastereotopic $\left.\mathrm{CH}_{2}\right) ; 1.97\left(\mathrm{~s}, 6 \mathrm{H},\left(\mathrm{CH}_{3}\right)_{2}\right) ; 0.16(\mathrm{~s}, 9 \mathrm{H}$, $\left.\mathrm{Si}\left(\mathrm{CH}_{3}\right)_{3}\right) .{ }^{7} \mathrm{Li} \mathrm{NMR}\left(\mathrm{C}_{6} \mathrm{D}_{6}, 194 \mathrm{MHz}, 295 \mathrm{~K}\right) \delta: 1.5 .{ }^{1} \mathrm{H}$ NMR $\left(\mathrm{THF}-\mathrm{d}_{8}, 500 \mathrm{MHz}, 295 \mathrm{~K}\right) \delta: 6.72\left(\mathrm{t},{ }^{3} \mathrm{~J}=7.9\right.$ $\mathrm{Hz}, 1 \mathrm{H}, \operatorname{ArH}) ; 6.68\left(\mathrm{~d},{ }^{3} \mathrm{~J}=7.1 \mathrm{~Hz}, 1 \mathrm{H}, \operatorname{ArH}\right) ; 6.51\left(\mathrm{~d},{ }^{3} \mathrm{~J}=7.7 \mathrm{~Hz}, 1 \mathrm{H}, \operatorname{ArH}\right) ; 6.00\left(\mathrm{t},{ }^{3} \mathrm{~J}=7.0 \mathrm{~Hz}, 1 \mathrm{H}, \operatorname{ArH}\right)$;

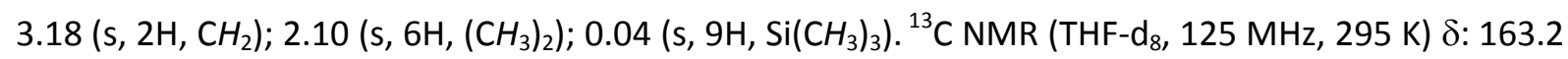
$\left(\mathrm{Ar}-\mathrm{NH}_{2}\right) ; 132.2(\mathrm{Ar}) ; 128.9(\mathrm{Ar}) ; 127.5\left(\mathrm{Ar}-\mathrm{CH}_{2}\right) ; 122.4(\mathrm{Ar}) ; 109.3(\mathrm{Ar}) ; 66.2\left(\mathrm{CH}_{2}\right) ; 45.8\left(\mathrm{CH}_{3}\right) ; 3.3$ $\left(\mathrm{Si}\left(\mathrm{CH}_{3}\right)_{3}\right) .{ }^{7} \mathrm{Li}$ NMR (THF-d $\left.8,194 \mathrm{MHz}, 295 \mathrm{~K}\right)$ ) : - 1.4.

\section{General procedure of preparation of lithium $N, N^{\prime}$-disubstituted-\{ $N^{\prime \prime}$-[2,6-di(propan-2- yl)phenyl]\}guanidinates $(2-3)$}

To a solution of lithium [2,6-di(propan-2-yl)phenyl]azanide (1) in $\mathrm{Et}_{2} \mathrm{O}$ cooled to $0{ }^{\circ} \mathrm{C}$, one equivalent of starting $N, N^{\prime}$-disubstituted carbodiimides (a solution in $\mathrm{Et}_{2} \mathrm{O}$ ) was added. Reaction mixtures were allowed to warm to the room temperature and stirred overnight. After that the $\mathrm{Et}_{2} \mathrm{O}$ was evaporated 
under vacuo and crude products were washed with small amount of hexane to give white crystalline of 2 - 3.

General procedure of preparation of lithium $N, N^{\prime}$-disubstituted-\{ $N^{\prime \prime}$-[2[(dimethylamino)methyl]phenyl\}guanidinates (8 - 9)

To a solution of lithium 2-[(dimethylamino)methyl]phenylazanide (7) in $\mathrm{Et}_{2} \mathrm{O}$ cooled to $0{ }^{\circ} \mathrm{C}$, one equivalent of starting $N, N^{\prime}$-disubstituted carbodiimides (a solution in $\mathrm{Et}_{2} \mathrm{O}$ ) was added. Reaction mixtures were allowed to warm to the room temperature and stirred overnight. After that the $\mathrm{Et}_{2} \mathrm{O}$ was evaporated under vacuo and crude products were washed with small amount of hexane to give white crystalline of $\mathbf{8} \mathbf{- 9}$.

General procedure of preparation of lithium $N, N^{\prime}$-disubstituted-\{ $N^{\prime \prime}$-trimethylsilyl-[2[(dimethylamino)methyl]phenyl\}guanidinates (13 - 14)

To a solution of lithium trimethylsilyl-2-[(dimethylamino)methyl]phenylazanide (12) in $\mathrm{Et}_{2} \mathrm{O}$ cooled to $0{ }^{\circ} \mathrm{C}$, one equivalent of starting $N, N^{\prime}$-disubstituted carbodiimides (a solution in $\mathrm{Et}_{2} \mathrm{O}$ ) was added. Reaction mixtures were allowed to warm to the room temperature and stirred overnight. After that the $\mathrm{Et}_{2} \mathrm{O}$ was evaporated under vacuo and crude products were washed with small amount of hexane to give white crystalline of $\mathbf{1 3} \mathbf{- 1 4}$.

\section{Preparation of 2}

$2.211 \mathrm{~g}(12.1 \mathrm{mmol})$ of $1,1.522 \mathrm{~g}$ (12.1 mmol) of $N, N^{\prime}$-[di(propan-2-yl)]carbodiimide, $20 \mathrm{ml} \mathrm{of} \mathrm{Et}{ }_{2} \mathrm{O}, 5$ $\mathrm{ml}$ of hexane. $2.922 \mathrm{~g}(70 \%)$ of white powder of $\mathbf{2}$. Single crystalline material suitable for XRD analyses were obtained under argon from saturated solution of $\mathbf{2}$ in $\mathrm{Et}_{2} \mathrm{O}$ cooled to $-30{ }^{\circ} \mathrm{C}$. M.p. 136$138^{\circ} \mathrm{C} .{ }^{1} \mathrm{H}$ NMR $\left(\mathrm{C}_{6} \mathrm{D}_{6}, 500 \mathrm{MHz}, 295 \mathrm{~K}\right)$ 8: $7.19(\mathrm{~s}, 2 \mathrm{H}, \operatorname{ArH}) ; 7.06(\mathrm{~s}, 1 \mathrm{H}, \operatorname{ArH}) ; 3.51(\mathrm{~m}, 2 \mathrm{H}, \mathrm{CH}) ; 3.45$ $\left(d,{ }^{3} \mathrm{~J}=9.6 \mathrm{~Hz}, 1 \mathrm{H}, \mathrm{NH}\right) ; 3.33(\mathrm{~m}, 1 \mathrm{H}, \mathrm{CH}) ; 3.27\left(\mathrm{q},{ }^{3} \mathrm{~J}=7.0 \mathrm{~Hz}, 2 \mathrm{H}, \mathrm{Et}_{2} \mathrm{O}\right) ; 3.21(\mathrm{~m}, 1 \mathrm{H}, \mathrm{CH}) ; 1.45\left(\mathrm{~d},{ }^{3} \mathrm{~J}=\right.$ $\left.6.9 \mathrm{~Hz}, 6 \mathrm{H}, \mathrm{CH}_{3}\right) ; 1.19\left(\mathrm{~d},{ }^{3} \mathrm{~J}=6.9 \mathrm{~Hz}, 6 \mathrm{H}, \mathrm{CH}_{3}\right) ; 1.15\left(\mathrm{~d},{ }^{3} \mathrm{~J}=6.1 \mathrm{~Hz}, 6 \mathrm{H}, \mathrm{CH}_{3}\right) ; 1.11\left(\mathrm{t},{ }^{3} \mathrm{~J}=7.0 \mathrm{~Hz}, 3 \mathrm{H}\right.$, $\left.\mathrm{Et}_{2} \mathrm{O}\right) ; 0.78\left(\mathrm{~d},{ }^{3} \mathrm{~J}=6.3 \mathrm{~Hz}, 6 \mathrm{H}, \mathrm{CH}_{3}\right) .{ }^{13} \mathrm{C} \mathrm{NMR}\left(\mathrm{C}_{6} \mathrm{D}_{6}, 125 \mathrm{MHz}, 295 \mathrm{~K}\right) \delta: 164.3(\mathrm{NCN}) ; 147.6$ (Ar-NH); 141.0 (Ar-CH); 123.7 (Ar); 120.9 (Ar); $67.2\left(\mathrm{Et}_{2} \mathrm{O}\right) ; 47.5(\mathrm{CH}) ; 44.4(\mathrm{CH}) ; 28.9(\mathrm{CH}) ; 27.1\left(\mathrm{CH}_{3}\right) ; 26.5$

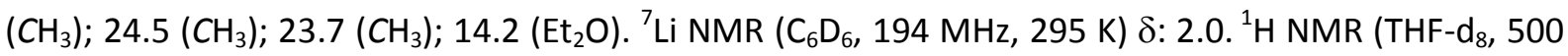
$\mathrm{MHz}, 295 \mathrm{~K}) \delta: 6.79\left(\mathrm{~d},{ }^{3} \mathrm{~J}=7.5 \mathrm{~Hz}, 2 \mathrm{H}, \mathrm{ArH}\right) ; 6.53\left(\mathrm{t},{ }^{3} \mathrm{~J}=7.5 \mathrm{~Hz}, 1 \mathrm{H}, \operatorname{ArH}\right) ; 3.55(\mathrm{~m}, 2 \mathrm{H}, \mathrm{CH}) ; 3.39$ (q, ${ }^{3} J$ $\left.=7.0 \mathrm{~Hz}, 2 \mathrm{H}, \mathrm{Et}_{2} \mathrm{O}\right) ; 3.36(\mathrm{~m}, 1 \mathrm{H}, \mathrm{CH}) ; 3.29\left(\mathrm{~d},{ }^{3} \mathrm{~J}=10.0 \mathrm{~Hz}, 1 \mathrm{H}, \mathrm{NH}\right) ; 3.00(\mathrm{~m}, 1 \mathrm{H}, \mathrm{CH}) ; 1.21\left(\mathrm{~d},{ }^{3} \mathrm{~J}=7.0\right.$ $\left.\mathrm{Hz}, 6 \mathrm{H}, \mathrm{CH}_{3}\right) ; 1.12\left(\mathrm{t},{ }^{3} \mathrm{~J}=7.0 \mathrm{~Hz}, 3 \mathrm{H}, \mathrm{Et}_{2} \mathrm{O}\right) ; 1.02\left(\mathrm{~d},{ }^{3} \mathrm{~J}=6.0 \mathrm{~Hz}, 6 \mathrm{H}, \mathrm{CH}_{3}\right) ; 0.98\left(\mathrm{~d},{ }^{3} \mathrm{~J}=6.8 \mathrm{~Hz}, 6 \mathrm{H}, \mathrm{CH}_{3}\right)$; $0.80\left(\mathrm{~d},{ }^{3} \mathrm{~J}=6.3 \mathrm{~Hz}, 6 \mathrm{H}, \mathrm{CH}_{3}\right) .{ }^{13} \mathrm{C}$ NMR (THF-d $\left.\mathrm{d}_{8}, 125 \mathrm{MHz}, 295 \mathrm{~K}\right) \delta: 162.8$ (NCN); 150.9 (Ar-NH); 141.1 
(Ar-CH); $122.9(\mathrm{Ar}) ; 118.2(\mathrm{Ar}) ; 66.9\left(\mathrm{Et}_{2} \mathrm{O}\right) ; 46.4(\mathrm{CH}) ; 44.5(\mathrm{CH}) ; 28.3(\mathrm{CH}) ; 27.2\left(\mathrm{CH}_{3}\right) ; 25.9\left(\mathrm{CH}_{3}\right) ; 24.5$ $\left(\mathrm{CH}_{3}\right) ; 23.6\left(\mathrm{CH}_{3}\right) ; 13.9\left(\mathrm{Et}_{2} \mathrm{O}\right) .{ }^{7} \mathrm{Li} \mathrm{NMR}\left(\mathrm{THF}-\mathrm{d}_{8}, 194 \mathrm{MHz}, 295 \mathrm{~K}\right) \delta:-0.6$.

\section{Preparation of 3}

$1.826 \mathrm{~g}(10.0 \mathrm{mmol})$ of $1,3.612 \mathrm{~g}$ (10.0 mmol) of $N, N^{\prime}$-bis[2,6-di(propan-2-yl)phenyl]carbodiimide, 15 $\mathrm{ml}$ of $\mathrm{Et}_{2} \mathrm{O}, 5 \mathrm{ml}$ of hexane. $4.847 \mathrm{~g}(78 \%)$ of white powder of 3 . Single crystalline material suitable for XRD analyses were obtained under argon from saturated solution of $\mathbf{3}$ in $\mathrm{Et}_{2} \mathrm{O}$ cooled to $-30{ }^{\circ} \mathrm{C}$. M.p. $154-156{ }^{\circ} \mathrm{C} .{ }^{1} \mathrm{H}$ NMR $\left(\mathrm{C}_{6} \mathrm{D}_{6}, 500 \mathrm{MHz}, 295 \mathrm{~K}\right)$ ): 7.09 (s, 8H, $\left.\operatorname{ArH}\right) ; 6.74(\mathrm{~s}, 1 \mathrm{H}, \operatorname{ArH}) ; 4.94(\mathrm{~s}, 1 \mathrm{H}$, $\mathrm{NH}) ; 3.77(\mathrm{~m}, 6 \mathrm{H}, \mathrm{CH}) ; 2.58\left(\mathrm{q},{ }^{3} \mathrm{~J}=7.0 \mathrm{~Hz}, 4 \mathrm{H}, \mathrm{Et}_{2} \mathrm{O}\right) ; 1.47$ (br s, 6H, $\left.\mathrm{CH}_{3}\right) ; 1.27$ (br s, $\left.18 \mathrm{H}, \mathrm{CH}_{3}\right) ; 1.09$ (br s, 6H, CH $) ; 1.03$ (br s, 6H, CH $\left.\mathrm{CH}_{3}\right) ; 0.38\left(\mathrm{t},{ }^{3} \mathrm{~J}=7.0 \mathrm{~Hz}, 6 \mathrm{H}, \mathrm{Et}_{2} \mathrm{O}\right) .{ }^{13} \mathrm{C} \mathrm{NMR}\left(\mathrm{C}_{6} \mathrm{D}_{6}, 125 \mathrm{MHz}, 295 \mathrm{~K}\right) \delta: 156.3$ (NCN); 147.2 (Ar); 138.3 (Ar); 126.4 (Ar); 123.2 (Ar); 65.2 (Et $\left.{ }_{2} \mathrm{O}\right) ; 29.2(\mathrm{CH}) ; 28.3(\mathrm{CH}) ; 26.7$ (br signal, $\mathrm{CH}_{3}$ ); 25.5 (br signal, $\left.\mathrm{CH}_{3}\right) ; 14.1\left(\mathrm{Et}_{2} \mathrm{O}\right)$; rest of signals remained hidden due to fast exchange processes of some fragment in the molecule. ${ }^{7} \mathrm{Li} \mathrm{NMR}\left(\mathrm{C}_{6} \mathrm{D}_{6}, 194 \mathrm{MHz}, 295 \mathrm{~K}\right) \delta:-1.0 .{ }^{1} \mathrm{H}$ NMR (THF- $\mathrm{d}_{8}$, $500 \mathrm{MHz}, 295 \mathrm{~K}) \delta: 7.08\left(\mathrm{~d},{ }^{3} \mathrm{~J}=7.6 \mathrm{~Hz}, 4 \mathrm{H}, \operatorname{ArH}\right) ; 6.97(\mathrm{~s}, 3 \mathrm{H}, \operatorname{ArH}) ; 6.86\left(\mathrm{t},{ }^{3} \mathrm{~J}=7.6 \mathrm{~Hz}, 2 \mathrm{H}, \operatorname{ArH}\right) ; 4.74$ $(\mathrm{s}, 1 \mathrm{H}, \mathrm{NH}) ; 3.65(\mathrm{~m}, 4 \mathrm{H}, \mathrm{CH}) ; 3.58(\mathrm{~m}, 2 \mathrm{H}, \mathrm{CH}) ; 3.39\left(\mathrm{q},{ }^{3} \mathrm{~J}=7.0 \mathrm{~Hz}, 4 \mathrm{H}, \mathrm{Et}_{2} \mathrm{O}\right) ; 1.11\left(\mathrm{~s}, 36 \mathrm{H}, \mathrm{CH}_{3}\right) ; 1.12$ $\left(\mathrm{t}^{3}{ }^{3}=7.0 \mathrm{~Hz}, 6 \mathrm{H}, \mathrm{Et}_{2} \mathrm{O}\right) .{ }^{13} \mathrm{C}$ NMR (THF-d $\mathrm{d}_{8}, 125 \mathrm{MHz}, 295 \mathrm{~K}$ ) $\delta: 156.4$ (br signal, NCN); 147.6 (Ar); 144.0 (br signal, Ar); 138.7 (Ar); 126.2 (Ar); 124.2 (br signal, Ar); 122.9 (Ar); 122.1 (br signal, Ar); 66.5 (Et $\mathrm{Et}_{2} \mathrm{O}$ ); $29.1(\mathrm{CH}) ; 28.7(\mathrm{CH}) ; 25.1$ (br signal, $\mathrm{CH}_{3}$ ); 24.6 (br signal, $\mathrm{CH}_{3}$ ); 15.9 (Et $\left.{ }_{2} \mathrm{O}\right)$. ${ }^{7} \mathrm{Li}$ NMR (THF-d $\mathrm{d}_{8}, 194 \mathrm{MHz}$, 295 K) $\delta:-2.8$

\section{Preparation of 4}

To a solution of $0.698 \mathrm{~g}(0.9 \mathrm{mmol})$ of 2 in $20 \mathrm{ml}$ of THF cooled to $-20{ }^{\circ} \mathrm{C}$, two equivalent of $\mathrm{n}$ butyllithium (1.6 M solution of hexanes) was added dropwise. Reaction mixture was allowed to warm to the room temperature and stirred overnight. The solution of $\mathbf{4}$ in THF was then left in the freezer at $-30{ }^{\circ} \mathrm{C}$ for a couple of days. $0.483 \mathrm{~g}(63 \%)$ of pure colourless single crystals of 4 suitable for XRD

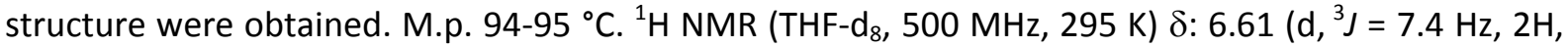
$\operatorname{ArH}) ; 6.19$ (br s, 1H, ArH); 3.58 (br s, 2H, CH); 3.53 (br s, 2H, CH); $1.09\left(\mathrm{~s}, 12 \mathrm{H}, \mathrm{CH}_{3}\right) ; 0.82\left(\mathrm{~s}, 12 \mathrm{H}, \mathrm{CH}_{3}\right)$. ${ }^{7}$ Li NMR (THF-d $\left.8,194 \mathrm{MHz}, 295 \mathrm{~K}\right)$ 8: -1.0.

\section{Preparation of $4 a$}

To a solution of $1.570 \mathrm{~g}(2.3 \mathrm{mmol})$ of 2 in $15 \mathrm{ml}$ of $\mathrm{Et}_{2} \mathrm{O}$ cooled to $-20{ }^{\circ} \mathrm{C}$, two equivalent of $\mathrm{n}$ butyllithium (1.6 M solution of hexanes) was added dropwise. Reaction mixture was allowed to warm to the room temperature and stirred overnight. After that the pale yellow $\mathrm{Et}_{2} \mathrm{O}$ solution of $4 \mathrm{a}$ was left 
in the freezer at $-30{ }^{\circ} \mathrm{C}$ for a couple of days. $1.178 \mathrm{~g} \mathrm{(67 \% )} \mathrm{of} \mathrm{pure} \mathrm{colourless} \mathrm{single} \mathrm{crystals} \mathrm{of} 4 \mathrm{a}$ suitable for XRD structure were obtained.

\section{Preparation of 5}

To a solution of $1.237 \mathrm{~g}(2.0 \mathrm{mmol})$ of 3 in $10 \mathrm{ml}$ of $\mathrm{Et}_{2} \mathrm{O}$ cooled to $-20{ }^{\circ} \mathrm{C}$, one equivalent of $\mathrm{n}$ butyllithium (1.6 M solution of hexanes) was added dropwise. Reaction mixture was allowed to warm to the room temperature and stirred overnight. After that the pale yellow $\mathrm{Et}_{2} \mathrm{O}$ solution of $\mathbf{5}$ was left in the freezer at $-30{ }^{\circ} \mathrm{C}$ for a couple of days. $0.893 \mathrm{~g}(64 \%)$ of pure colourless single crystals of 5 suitable for XRD structure were obtained. M.p. 149-151 ${ }^{\circ} \mathrm{C} .{ }^{1} \mathrm{H}$ NMR (THF-d $\left.8,500 \mathrm{MHz}, 295 \mathrm{~K}\right)$ 8: 7.18 (s, 2H, ArH); 6.92 (br s, 5H, ArH); 6.63 (s, 2H, ArH); 4.05 (s, 2H, CH); 3.87 (br s, 4H, CH); 3.39 (q, ${ }^{3}$ J = 7.1 $\mathrm{Hz}, 8 \mathrm{H}, \mathrm{Et}_{2} \mathrm{O}$ ); $1.14\left(\mathrm{t}^{3}{ }^{3}=7.0 \mathrm{~Hz}, 12 \mathrm{H}, \mathrm{Et}_{2} \mathrm{O}\right.$ ); 1.08 (br s, 36H, $\mathrm{CH}_{3}$ ). ${ }^{7} \mathrm{Li} \mathrm{NMR}\left(\mathrm{THF}^{\mathrm{d}} \mathrm{d}_{8}, 194 \mathrm{MHz}, 295 \mathrm{~K}\right) \delta$ : -2.6 .

\section{Preparation of 6. $\left[\mathrm{Et}_{2} \mathrm{O}\right]_{2}$}

$0.889 \mathrm{~g}$ ( $3.7 \mathrm{mmol}$ ) of 6 was dissolved in $10 \mathrm{ml}$ of $\mathrm{Et}_{2} \mathrm{O}$. The colorless solution was left in the freezer at $-30{ }^{\circ} \mathrm{C}$. After several days colourless single crystals of $6 .\left[\mathrm{Et}_{2} \mathrm{O}\right]_{2}$ suitable for XRD structure were obtained in almost quantitative yield. M.p. $104-105^{\circ} \mathrm{C} .{ }^{1} \mathrm{H}$ NMR (THF-d $\left.\mathrm{d}_{8}, 500 \mathrm{MHz}, 295 \mathrm{~K}\right) \delta: 6.71\left(\mathrm{~d},{ }^{3} \mathrm{~J}\right.$ $=7.4 \mathrm{~Hz}, 2 \mathrm{H}, \mathrm{ArH}) ; 6.33\left(\mathrm{t},{ }^{3} \mathrm{~J}=7.4 \mathrm{~Hz}, 1 \mathrm{H}, \operatorname{ArH}\right) ; 4.06(\mathrm{~m}, 2 \mathrm{H}, \mathrm{CH}) ; 1.05\left(\mathrm{~d},{ }^{3} \mathrm{~J}=7.0 \mathrm{~Hz}, 12 \mathrm{H}, \mathrm{CH}_{3}\right) ;-0.08$ (s, 9H, Si( $\left.\left.\mathrm{CH}_{3}\right)_{3}\right) .{ }^{7} \mathrm{Li}$ NMR (THF-d $\left.8,194 \mathrm{MHz}, 295 \mathrm{~K}\right)$ 8: -0.2.

\section{Preparation of $\left[\text { 7.Et }{ }_{2} \mathrm{O}\right]_{2}$}

$0.652 \mathrm{~g}(4.2 \mathrm{mmol})$ of 7 was dissolved in $5 \mathrm{ml}$ of $\mathrm{Et}_{2} \mathrm{O}$. The colorless solution was left in the freezer at $-30{ }^{\circ} \mathrm{C}$. After several days colourless single crystals of [7.Et $\left.{ }_{2} \mathrm{O}\right]_{2}$ suitable for XRD structure were obtained in almost quantitative yield. M.p. $75-76{ }^{\circ} \mathrm{C} .{ }^{1} \mathrm{H} N M R\left(\mathrm{C}_{6} \mathrm{D}_{6}, 500 \mathrm{MHz}, 295 \mathrm{~K}\right) \delta: 7.14\left(\mathrm{t},{ }^{3} \mathrm{~J}=\right.$ $7.1 \mathrm{~Hz}, 1 \mathrm{H}, \operatorname{ArH}) ; 6.84\left(\mathrm{~d},{ }^{3} \mathrm{~J}=7.1 \mathrm{~Hz}, 1 \mathrm{H}, \operatorname{ArH}\right) ; 6.57\left(\mathrm{~d},{ }^{3} \mathrm{~J}=7.9 \mathrm{~Hz}, 1 \mathrm{H}, \operatorname{ArH}\right) ; 6.48\left(\mathrm{t},{ }^{3} \mathrm{~J}=7.2 \mathrm{~Hz}, 1 \mathrm{H}\right.$, $\operatorname{ArH}) ; 3.17\left(\mathrm{q},{ }^{3} \mathrm{~J}=7.0 \mathrm{~Hz}, 2 \mathrm{H}, \mathrm{Et}_{2} \mathrm{O}\right) ; 3.10\left(\mathrm{br} \mathrm{s}, 2 \mathrm{H}, \mathrm{CH}_{2}\right) ; 2.79(\mathrm{~s}, 1 \mathrm{H}, \mathrm{NH}) ; 1.82\left(\mathrm{~s}, 6 \mathrm{H},\left(\mathrm{CH}_{3}\right)_{2}\right) ; 1.01\left(\mathrm{t},{ }^{3} \mathrm{~J}\right.$ $\left.=6.9 \mathrm{~Hz}, 3 \mathrm{H}, \mathrm{Et}_{2} \mathrm{O}\right) .{ }^{7} \mathrm{Li} \mathrm{NMR}\left(\mathrm{C}_{6} \mathrm{D}_{6}, 194 \mathrm{MHz}, 295 \mathrm{~K}\right) \delta: 1.6$.

\section{Preparation of 8}

$0.776 \mathrm{~g}(5.0 \mathrm{mmol})$ of $7,0.627 \mathrm{~g}(4.9 \mathrm{mmol})$ of $N, N^{\prime}$-[di(propan-2-yl)]carbodiimide, $10 \mathrm{ml} \mathrm{of} \mathrm{Et} \mathrm{O}_{2} \mathrm{O}, 5 \mathrm{ml}$ of hexane. $0.935 \mathrm{~g}(67 \%)$ of white powder of $\mathbf{8}$. Single crystalline material suitable for XRD analyses were obtained under argon from saturated solution of 8 in $\mathrm{Et}_{2} \mathrm{O}$ cooled to $-30^{\circ} \mathrm{C}$. M.p. $158-160{ }^{\circ} \mathrm{C} .{ }^{1} \mathrm{H}$ $\operatorname{NMR}\left(\mathrm{C}_{6} \mathrm{D}_{6}, 500 \mathrm{MHz}, 295 \mathrm{~K}\right) \delta: 7.16\left(\mathrm{t},{ }^{3} \mathrm{~J}=6.9 \mathrm{~Hz}, 1 \mathrm{H}, \operatorname{ArH}\right) ; 6.92\left(\mathrm{~d},{ }^{3} \mathrm{~J}=8.2 \mathrm{~Hz}, 1 \mathrm{H}, \operatorname{ArH}\right) ; 6.88\left(\mathrm{~d},{ }^{3} \mathrm{~J}=\right.$ $7.3 \mathrm{~Hz}, 1 \mathrm{H}, \mathrm{ArH}$ ); $6.54\left(\mathrm{t},{ }^{3} \mathrm{~J}=7.2 \mathrm{~Hz}, 1 \mathrm{H}, \mathrm{ArH}\right.$ ); $3.54\left(\mathrm{~m}, 1 \mathrm{H}, \mathrm{CH}\right.$ ); 3.51, 2.82 (AX spin system, ${ }^{2} \mathrm{~J}=11.7$ 
$\mathrm{Hz}, 2 \mathrm{H}$, diastereotopic $\left.\mathrm{CH}_{2}\right) ; 3.25(\mathrm{~m}, 1 \mathrm{H}, \mathrm{CH}) ; 2.11\left(\mathrm{~s}, 3 \mathrm{H}, \mathrm{NCH}_{3}\right) ; 1.58\left(\mathrm{~s}, 3 \mathrm{H}, \mathrm{NCH}_{3}\right) ; 1.21\left(\mathrm{~d},{ }^{2} \mathrm{~J}=6.2\right.$ $\left.\mathrm{Hz}, 3 \mathrm{H}, \mathrm{CH}_{3}\right) ; 1.18\left(\mathrm{~d},{ }^{2} \mathrm{~J}=6.2 \mathrm{~Hz}, 3 \mathrm{H}, \mathrm{CH}_{3}\right) ; 1.07\left(\mathrm{~d},{ }^{2} \mathrm{~J}=6.1 \mathrm{~Hz}, 3 \mathrm{H}, \mathrm{CH}_{3}\right) ; 0.75\left(\mathrm{~d},{ }^{2} \mathrm{~J}=6.4 \mathrm{~Hz}, 3 \mathrm{H}, \mathrm{CH}_{3}\right)$. ${ }^{13} \mathrm{C} \mathrm{NMR}\left(\mathrm{C}_{6} \mathrm{D}_{6}, 125 \mathrm{MHz}, 295 \mathrm{~K}\right) \delta$ : 162.4 (NCN); 157.5 (Ar-NH); 132.7 (Ar); $129.3(\mathrm{Ar}) ; 124.5\left(\mathrm{Ar}-\mathrm{CH}_{2}\right)$; 118.0 (Ar); $114.3(\mathrm{Ar}) ; 65.3\left(\mathrm{CH}_{2}\right) ; 46.8(\mathrm{CH}) ; 46.3\left(\mathrm{NCH}_{3}\right) ; 44.1\left(\mathrm{NCH}_{3}\right) ; 43.4(\mathrm{CH}) ; 26.3\left(\mathrm{CH}_{3}\right) ; 25.8$ $\left(\mathrm{CH}_{3}\right) ; 25.6\left(\mathrm{CH}_{3}\right) ; 23.4\left(\mathrm{CH}_{3}\right) .{ }^{7} \mathrm{Li} \mathrm{NMR}\left(\mathrm{C}_{6} \mathrm{D}_{6}, 194 \mathrm{MHz}, 295 \mathrm{~K}\right)$ \&: 2.0. ${ }^{1} \mathrm{H}$ NMR (THF-d $\left., 500 \mathrm{MHz}, 295 \mathrm{~K}\right)$ $\delta: 6.85\left(\mathrm{t},{ }^{3} \mathrm{~J}=6.9 \mathrm{~Hz}, 1 \mathrm{H}, \operatorname{ArH}\right) ; 6.73\left(\mathrm{~d},{ }^{3} \mathrm{~J}=6.7 \mathrm{~Hz}, 1 \mathrm{H}, \operatorname{ArH}\right) ; 6.88\left(\mathrm{~d},{ }^{3} \mathrm{~J}=8.2 \mathrm{~Hz}, 1 \mathrm{H}, \operatorname{ArH}\right) ; 6.21\left(\mathrm{t},{ }^{3} \mathrm{~J}=\right.$ $7.2 \mathrm{~Hz}, 1 \mathrm{H}, \operatorname{ArH}) ; 4.00\left(\mathrm{~d},{ }^{3} \mathrm{~J}=9.2 \mathrm{~Hz}, 1 \mathrm{H}, \mathrm{NH}\right.$ ); 3.48, $2.82\left(\mathrm{AX}\right.$ spin system, ${ }^{2} \mathrm{~J}=11.7 \mathrm{~Hz}, 2 \mathrm{H}$, diastereotopic $\left.\mathrm{CH}_{2}\right) ; 3.41(\mathrm{~m}, 1 \mathrm{H}, \mathrm{CH}) ; 3.36(\mathrm{~m}, 1 \mathrm{H}, \mathrm{CH}) ; 2.21\left(\mathrm{br} \mathrm{s}, 3 \mathrm{H}, \mathrm{NCH}_{3}\right) ; 1.52\left(\mathrm{br} \mathrm{s}, 3 \mathrm{H}, \mathrm{NCH}_{3}\right)$; $1.11\left(\mathrm{~m}, 9 \mathrm{H}, \mathrm{CH}_{3}\right) ; 0.84\left(\mathrm{~d},{ }^{2} \mathrm{~J}=6.6 \mathrm{~Hz}, 3 \mathrm{H}, \mathrm{CH}_{3}\right) .{ }^{13} \mathrm{C} \mathrm{NMR}\left(\mathrm{THF}-\mathrm{d}_{8}, 125 \mathrm{MHz}, 295 \mathrm{~K}\right) \delta: 162.9$ (NCN); $157.9(\mathrm{Ar}-\mathrm{NH}) ; 132.6(\mathrm{Ar}) ; 129.2(\mathrm{Ar}) ; 124.6\left(\mathrm{Ar}-\mathrm{CH}_{2}\right)$; $118.0(\mathrm{Ar}) ; 113.9(\mathrm{Ar}) ; 65.6\left(\mathrm{CH}_{2}\right) ; 47.1(\mathrm{CH}) ; 46.4$ $\left(\mathrm{NCH}_{3}\right) ; 44.5\left(\mathrm{NCH}_{3}\right) ; 43.6(\mathrm{CH}) ; 26.4\left(\mathrm{CH}_{3}\right) ; 26.0\left(\mathrm{CH}_{3}\right) ; 25.7\left(\mathrm{CH}_{3}\right) ; 23.4\left(\mathrm{CH}_{3}\right) .{ }^{7} \mathrm{Li}$ NMR $\left(\mathrm{THF}-\mathrm{d}_{8}, 194\right.$ $\mathrm{MHz}, 295 \mathrm{~K})$ 8: 2.0.

\section{Preparation of 9}

$1.253 \mathrm{~g}(8 \mathrm{mmol})$ of 7, $2.912 \mathrm{~g}(8 \mathrm{mmol})$ of $N, N^{\prime}$-bis[2,6-di(propan-2-yl)phenyl]carbodiimide, $20 \mathrm{ml}$ of $\mathrm{Et}_{2} \mathrm{O}, 2 \times 5 \mathrm{ml}$ of hexane. $3.025 \mathrm{~g}$ (64\%) of white powder of 9. Single crystalline material suitable for $\mathrm{XRD}$ analyses were obtained under argon from saturated solution of 9 in $\mathrm{Et}_{2} \mathrm{O}$ cooled to $-30{ }^{\circ} \mathrm{C}$. M.p. 148-150 ${ }^{\circ} \mathrm{C} .{ }^{1} \mathrm{H}$ NMR $\left(\mathrm{C}_{6} \mathrm{D}_{6}, 500 \mathrm{MHz}, 295 \mathrm{~K}\right) \delta: 7.92\left(\mathrm{~d},{ }^{3} \mathrm{~J}=8.2 \mathrm{~Hz}, 1 \mathrm{H}, \mathrm{ArH}\right) ; 7.35-7.12(\mathrm{~m}, 4 \mathrm{H}, \operatorname{ArH})$; $7.02\left(\mathrm{~d},{ }^{3} \mathrm{~J}=7.6 \mathrm{~Hz}, 2 \mathrm{H}, \operatorname{ArH}\right) ; 6.86\left(\mathrm{~d},{ }^{3} \mathrm{~J}=7.3 \mathrm{~Hz}, 1 \mathrm{H}, \operatorname{ArH}\right) ; 6.70\left(\mathrm{t},{ }^{3} \mathrm{~J}=7.5 \mathrm{~Hz}, 1 \mathrm{H}, \operatorname{ArH}\right) ; 6.65\left(\mathrm{t},{ }^{3} \mathrm{~J}=\right.$ $7.3 \mathrm{~Hz}, 1 \mathrm{H}, \mathrm{ArH}) ; 5.92(\mathrm{~s}, 1 \mathrm{H}, \mathrm{NH}) ; 3.91$ (br s, 2H, CH); $3.84(\mathrm{~m}, 2 \mathrm{H}, \mathrm{CH}), 2.92\left(\mathrm{br} \mathrm{s}, 2 \mathrm{H}, \mathrm{CH}_{2}\right) ; 1.38$ (s, $\left.6 \mathrm{H}, \mathrm{N}\left(\mathrm{CH}_{3}\right)_{2}\right) ; 1.22\left(\mathrm{~d},{ }^{2} \mathrm{~J}=6.8 \mathrm{~Hz}, 12 \mathrm{H}, \mathrm{CH}_{3}\right) ; 1.12\left(\mathrm{~d},{ }^{2} \mathrm{~J}=6.8 \mathrm{~Hz}, 12 \mathrm{H}, \mathrm{CH}_{3}\right) .{ }^{13} \mathrm{C} \mathrm{NMR}\left(\mathrm{C}_{6} \mathrm{D}_{6}, 125 \mathrm{MHz}\right.$, $295 \mathrm{~K}) \delta: 157.6(\mathrm{Ar}-\mathrm{NH}) ; 153.6\left(\mathrm{Ar}^{\mathrm{Dipp}}-\mathrm{N}\right) ; 152.6\left(\mathrm{Ar}^{\mathrm{Dipp}}-\mathrm{N}\right) ; 143.3\left(\mathrm{Ar}^{\mathrm{Dipp}}-\mathrm{CH}\right) ; 137.7\left(\mathrm{Ar}^{\mathrm{Dipp}}-\mathrm{CH}\right) ; 133.1$ (Ar); 130.5 (Ar); 127.1 (Ar); $126.3\left(\mathrm{Ar}-\mathrm{CH}_{2}\right) ; 124.3$ (Ar); 123.5 (Ar); 120.8 (Ar); 119.7 (Ar); $116.2(\mathrm{Ar})$; $64.6\left(\mathrm{CH}_{2}\right) ; 43.8$ (br s, $\left.\mathrm{NCH}_{3}\right) ; 29.4(\mathrm{CH}) ; 27.9(\mathrm{CH}) ; 25.0\left(\mathrm{CH}_{3}\right) ; 24.2\left(\mathrm{CH}_{3}\right)$; signal of central carbon atom from NCN fragment remained hidden due to fast exchange processes (of some fragment in the

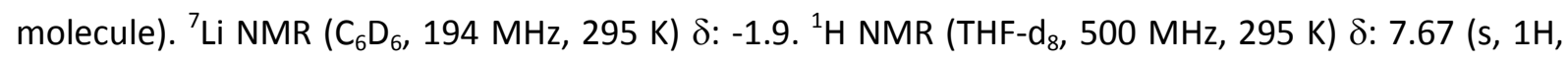
$\operatorname{ArH}) ; 6.89-6.78(\mathrm{~m}, 4 \mathrm{H}, \mathrm{ArH}) ; 6.64-6.59(\mathrm{~m}, 3 \mathrm{H}, \mathrm{ArH}) ; 6.54\left(\mathrm{~d},{ }^{3} \mathrm{~J}=7.0 \mathrm{~Hz}, 1 \mathrm{H}, \operatorname{ArH}\right) ; 6.29\left(\mathrm{t},{ }^{3} \mathrm{~J}=7.4\right.$ $\mathrm{Hz}, 1 \mathrm{H}, \mathrm{ArH}) ; 3.75(\mathrm{~m}, 4 \mathrm{H}, \mathrm{CH}) ; 3.00\left(\mathrm{~s}, 2 \mathrm{H}, \mathrm{CH}_{2}\right) ; 1.57\left(\mathrm{~s}, 6 \mathrm{H}, \mathrm{N}\left(\mathrm{CH}_{3}\right)_{2}\right) ; 1.14\left(\mathrm{~d},{ }^{3} \mathrm{~J}=7.0 \mathrm{~Hz}, 12 \mathrm{H}, \mathrm{CH}_{3}\right)$; $1.10\left(\mathrm{~d},{ }^{2} \mathrm{~J}=6.8 \mathrm{~Hz}, 12 \mathrm{H}, \mathrm{CH}_{3}\right) .{ }^{13} \mathrm{C}$ NMR (THF-d $\left., 125 \mathrm{MHz}, 295 \mathrm{~K}\right) \delta: 157.2(\mathrm{Ar}-\mathrm{NH}) ; 148.1\left(\mathrm{Ar}^{\mathrm{Dipp}}-\mathrm{N}\right)$; 142.2 ( $\left.\mathrm{Ar}^{\text {Dipp }}-\mathrm{CH}\right) ; 129.6(\mathrm{Ar}) ; 127.7(\mathrm{Ar}) ; 124.4\left(\mathrm{Ar}-\mathrm{CH}_{2}\right) ; 123.2(\mathrm{Ar}) ; 120.6(\mathrm{Ar}) ; 119.6(\mathrm{Ar}) ; 118.6(\mathrm{Ar})$; $64.3\left(\mathrm{CH}_{2}\right) ; 45.3$ (br s, NCH$) ; 28.5(\mathrm{CH}) ; 25.7\left(\mathrm{CH}_{3}\right) ; 24.1\left(\mathrm{CH}_{3}\right)$; signal of central carbon atom from $\mathrm{NCN}$ fragment remained hidden due to fast exchange processes (of some fragment in the molecule). ${ }^{7}$ Li NMR (THF-d 8,194 MHz, 295 K) \&: 1.5. 


\section{Preparation of 10}

To a solution of $1.068 \mathrm{~g}(1.9 \mathrm{mmol})$ of 8 in $15 \mathrm{ml}$ of $\mathrm{Et}_{2} \mathrm{O}$ cooled to $-20{ }^{\circ} \mathrm{C}$, two equivalent of $\mathrm{n}$ butyllithium (1.6 M solution of hexanes) was added dropwise. Reaction mixture was allowed to warm to the room temperature and stirred overnight. After that the $\mathrm{Et}_{2} \mathrm{O}$ was evaporated under vacuo and the obtained pale yellow crude product of $\mathbf{1 0}$ was dissolved in $10 \mathrm{ml}$ of THF. The solution of $\mathbf{1 0}$ in THF was then left in the freezer at $-30{ }^{\circ} \mathrm{C}$ for a couple of days. $1.125 \mathrm{~g}$ (69\%) of pure colourless single crystals of 10 suitable for XRD structure were obtained. M.p. $125-126.5^{\circ} \mathrm{C}$. Insoluble in THF- $\mathrm{d}_{8}, \mathrm{C}_{6} \mathrm{D}_{6}$ and $\mathrm{C}_{6} \mathrm{D}_{12}$.

\section{Preparation of 11}

To a solution of $0.523 \mathrm{~g}(0.9 \mathrm{mmol})$ of 9 in $10 \mathrm{ml}$ of $\mathrm{Et}_{2} \mathrm{O}$ cooled to $-20{ }^{\circ} \mathrm{C}$, one equivalent of butyllithium (1.6 M solution of hexanes) was added dropwise. Reaction mixture was allowed to warm to the room temperature and stirred overnight. After that the pale yellow $\mathrm{Et}_{2} \mathrm{O}$ solution of $\mathbf{1 1}$ was left in the freezer at $-30{ }^{\circ} \mathrm{C}$ for a couple of days. $0.346 \mathrm{~g}(75 \%)$ of pure colourless single crystals of 11 suitable for XRD structure were obtained. M.p. $85-87^{\circ} \mathrm{C}$. ${ }^{1} \mathrm{H}$ NMR (THF-d $\left.8,500 \mathrm{MHz}, 295 \mathrm{~K}\right) \delta: 6.80$ (s, $4 \mathrm{H}, \operatorname{ArH}) ; 6.61\left(\mathrm{~d},{ }^{3} \mathrm{~J}=8.2 \mathrm{~Hz}, 1 \mathrm{H}, \operatorname{ArH}\right) ; 6.49(\mathrm{~s}, 2 \mathrm{H}, \operatorname{ArH}) ; 6.40\left(\mathrm{t},{ }^{3} \mathrm{~J}=7.6 \mathrm{~Hz}, 1 \mathrm{H}, \operatorname{ArH}\right) ; 6.34\left(\mathrm{~d},{ }^{3} \mathrm{~J}=7.2\right.$ $\mathrm{Hz}, 1 \mathrm{H}, \mathrm{ArH}) ; 5.79\left(\mathrm{t},{ }^{3} \mathrm{~J}=7.2 \mathrm{~Hz}, 1 \mathrm{H}, \mathrm{ArH}\right) ; 3.97(\mathrm{~m}, 4 \mathrm{H}, \mathrm{CH}) ; 2.97\left(\mathrm{br} \mathrm{s}, 2 \mathrm{H}, \mathrm{CH}_{2}\right) ; 1.83\left(\mathrm{~s}, 6 \mathrm{H}, \mathrm{N}\left(\mathrm{CH}_{3}\right)_{2}\right)$; $1.17\left(\mathrm{~d},{ }^{3} \mathrm{~J}=5.3 \mathrm{~Hz}, 12 \mathrm{H}, \mathrm{CH}_{3}\right) ; 1.10\left(\mathrm{~d},{ }^{2} \mathrm{~J}=6.8 \mathrm{~Hz}, 12 \mathrm{H}, \mathrm{CH}_{3}\right) .{ }^{7} \mathrm{Li} \mathrm{NMR}\left(\mathrm{THF}-\mathrm{d}_{8}, 194 \mathrm{MHz}, 295 \mathrm{~K}\right) \delta:-0.2$ $(1 \mathrm{Li}) ;-3.0(1 \mathrm{Li})$.

\section{Preparation of $[12]_{2}$}

$0.621 \mathrm{~g}$ ( $2.7 \mathrm{mmol})$ of $\mathbf{1 2}$ was dissolved in $5 \mathrm{ml}$ of hexane. The colorless solution was left in the freezer at $-30^{\circ} \mathrm{C}$. After several days colourless single crystals of [12] $]_{2}$ suitable for XRD structure were obtained in almost quantitative yield. M.p. $<50^{\circ} \mathrm{C}$.

\section{Preparation of 13}

$0.986 \mathrm{~g}(4.3 \mathrm{mmol})$ of $12,0.545 \mathrm{~g}(4.3 \mathrm{mmol})$ of $N, N^{\prime}$-[di(propan-2-yl)]carbodiimide, $15 \mathrm{ml}$ of $\mathrm{Et}_{2} \mathrm{O}, 5$ $\mathrm{ml}$ of hexane. $0.735 \mathrm{~g}$ (76 \%) of white powder of 13. M.p. $158-159.5^{\circ} \mathrm{C} .{ }^{1} \mathrm{H} N M R\left(\mathrm{C}_{6} \mathrm{D}_{6}, 500 \mathrm{MHz}, 295\right.$ K) $\delta: 7.13\left(\mathrm{t},{ }^{3} \mathrm{~J}=8.1 \mathrm{~Hz}, 1 \mathrm{H}, \operatorname{ArH}\right) ; 6.86\left(\mathrm{~d},{ }^{3} \mathrm{~J}=7.3 \mathrm{~Hz}, 1 \mathrm{H}, \operatorname{ArH}\right) ; 6.68-6.63(\mathrm{~m}, 2 \mathrm{H}, \operatorname{ArH}) ; 3.92,2.58$ (AX spin system, ${ }^{2} J=10.3 \mathrm{~Hz}, 2 \mathrm{H}$, diastereotopic $\mathrm{CH}_{2}$ ); $3.87(\mathrm{~m}, 1 \mathrm{H}, \mathrm{CH}) ; 3.33(\mathrm{~m}, 1 \mathrm{H}, \mathrm{CH}) ; 2.24$ (br s, $\left.3 \mathrm{H}, \mathrm{NCH}_{3}\right) ; 1.49$ (br s, 3H, NCH 3$) ; 1.32\left(\mathrm{~s}, 6 \mathrm{H}, \mathrm{CH}_{3}\right) ; 1.15\left(\mathrm{~s}, 6 \mathrm{H}, \mathrm{CH}_{3}\right) ; 0.38\left(\mathrm{~s}, 9 \mathrm{H}, \mathrm{Si}\left(\mathrm{CH}_{3}\right)_{3}\right) .{ }^{13} \mathrm{C} \mathrm{NMR}$ $\left(\mathrm{C}_{6} \mathrm{D}_{6}, 125 \mathrm{MHz}, 295 \mathrm{~K}\right)$ \&: $166.4(\mathrm{NCN}) ; 157.2(\mathrm{Ar}-\mathrm{NH}) ; 132.8(\mathrm{Ar}) ; 128.5(\mathrm{Ar}) ; 127.0\left(\mathrm{Ar}^{-\mathrm{CH}_{2}}\right) ; 124.2$ (Ar); $116.5(\mathrm{Ar}) ; 65.2\left(\mathrm{CH}_{2}\right) ; 50.1(\mathrm{CH}) ; 47.3\left(\mathrm{NCH}_{3}\right) ; 26.8\left(\mathrm{CH}_{3}\right) ; 26.5\left(\mathrm{CH}_{3}\right) ; 25.9\left(\mathrm{CH}_{3}\right) ; 24.1\left(\mathrm{CH}_{3}\right) ; 3.58$

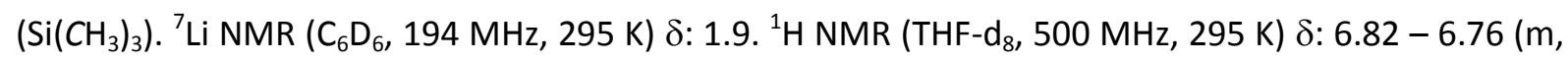


$2 \mathrm{H}, \operatorname{ArH}) ; 6.53\left(\mathrm{~d},{ }^{3} \mathrm{~J}=7.9 \mathrm{~Hz}, 1 \mathrm{H}, \operatorname{ArH}\right) ; 6.18\left(\mathrm{t},{ }^{3} \mathrm{~J}=7.2 \mathrm{~Hz}, 1 \mathrm{H}, \operatorname{ArH}\right) ; 3.73(\mathrm{~m}, 2 \mathrm{H}, \mathrm{CH}) ; 3.23(\mathrm{~s}, 2 \mathrm{H}$, $\left.\mathrm{CH}_{2}\right) ; 2.15\left(\mathrm{~s}, 6 \mathrm{H}, \mathrm{NCH}_{3}\right) ; 0.92\left(\mathrm{~d},{ }^{3} \mathrm{~J}=6.4 \mathrm{~Hz}, 12 \mathrm{H}, \mathrm{CH}_{3}\right) ; 0.22\left(\mathrm{~s}, 9 \mathrm{H}, \mathrm{Si}\left(\mathrm{CH}_{3}\right)_{3}\right) .{ }^{13} \mathrm{C}$ NMR $\left(\mathrm{THF}-\mathrm{d}_{8}, 125\right.$

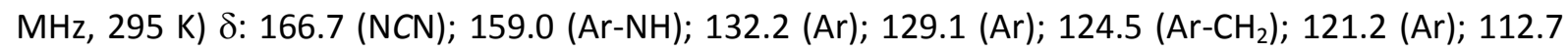
(Ar); $66.0\left(\mathrm{CH}_{2}\right) ; 46.6(\mathrm{CH}) ; 45.9\left(\mathrm{NCH}_{3}\right) ; 25.4\left(\mathrm{CH}_{3}\right) ; 7.6\left(\mathrm{Si}\left(\mathrm{CH}_{3}\right)_{3}\right) .{ }^{7} \mathrm{Li} \mathrm{NMR}\left(\mathrm{THF}-\mathrm{d}_{8}, 194 \mathrm{MHz}, 295 \mathrm{~K}\right) \delta$ : -1.5 .

\section{Preparation of 14}

$0.587 \mathrm{~g}$ (2.6 mmol) of 12, $0.932 \mathrm{~g}$ (2.6 mmol) of $N, N^{\prime}$-bis[2,6-di(propan-2-yl)phenyl]carbodiimide, 10 $\mathrm{ml}$ of $\mathrm{Et}_{2} \mathrm{O}, 5 \mathrm{ml}$ of hexane. $1.115 \mathrm{~g} \mathrm{(73 \% )} \mathrm{of} \mathrm{white} \mathrm{powder} \mathrm{of} \mathrm{14.} \mathrm{M.p.} \mathrm{205-207}{ }^{\circ} \mathrm{C}$. Single crystalline material suitable for XRD analyses were obtained under argon from saturated solution of 14 in $\mathrm{Et}_{2} \mathrm{O}$ cooled to $-30{ }^{\circ} \mathrm{C}^{1} \mathrm{H}$ NMR $\left(\mathrm{C}_{6} \mathrm{D}_{6}, 500 \mathrm{MHz}, 295 \mathrm{~K}\right) \delta: 7.03(\mathrm{br} \mathrm{s}, 3 \mathrm{H}, \mathrm{ArH}) ; 6.95\left(\mathrm{t},{ }^{3} \mathrm{~J}=8.2 \mathrm{~Hz}, 1 \mathrm{H}, \operatorname{ArH}\right)$; $6.87\left(\mathrm{~d},{ }^{3} \mathrm{~J}=8.1 \mathrm{~Hz}, 2 \mathrm{H}, \operatorname{ArH}\right) ; 6.85-6.75(\mathrm{~m}, 3 \mathrm{H}, \operatorname{ArH}) ; 6.51\left(\mathrm{~d},{ }^{3} \mathrm{~J}=7.4 \mathrm{~Hz}, 1 \mathrm{H}, \operatorname{ArH}\right) ; 6.35\left(\mathrm{t},{ }^{3} \mathrm{~J}=7.3\right.$ $\mathrm{Hz}, 1 \mathrm{H}, \mathrm{ArH}$ ); 4.09, 2.17 (br s, AX spin system, $2 \mathrm{H}$, diastereotopic $\mathrm{CH}_{2}$ ); 3.61 (br s, $\left.4 \mathrm{H}, \mathrm{CH}\right), 1.24$ (br s,

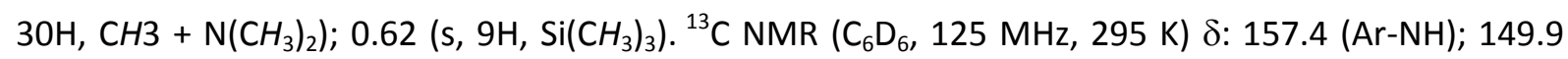
$\left(\mathrm{Ar}^{\mathrm{Dipp}}-\mathrm{N}\right) ; 131.3(\mathrm{Ar}) ; 128.7$ (Ar); 124.1 (br s, $\left.\mathrm{Ar}\right) ; 122.9(\mathrm{Ar}) ; 122.5\left(\mathrm{Ar}-\mathrm{CH}_{2}\right) ; 116.3(\mathrm{Ar}) ; 64.0\left(\mathrm{CH}_{2}\right)$; 45.6 (s, $\left.\mathrm{NCH}_{3}\right) ; 29.0(\mathrm{CH}) ; 28.4(\mathrm{CH}) ; 25.7$ (br s, $\left.\mathrm{CH}_{3}\right) ; 23.8$ (br s, $\left.\mathrm{CH}_{3}\right) ; 3.1\left(\mathrm{Si}\left(\mathrm{CH}_{3}\right)_{3}\right)$; rest of signals remained hidden due to fast exchange processes of some fragment in the molecule. ${ }^{7} \mathrm{Li} N M R\left(\mathrm{C}_{6} \mathrm{D}_{6}\right.$, $194 \mathrm{MHz}, 295 \mathrm{~K}) \delta:-2.1 .{ }^{1} \mathrm{H}$ NMR (THF-d $\left.8,500 \mathrm{MHz}, 295 \mathrm{~K}\right) \delta: 7.00$ (br s, 6H, ArH); $6.49-6.41$ (m, 3H, $\mathrm{ArH}) ; 6.02\left(\mathrm{t},{ }^{3} \mathrm{~J}=7.2 \mathrm{~Hz}, 1 \mathrm{H}, \mathrm{ArH}\right) ; 3.68(\mathrm{br} \mathrm{s}, 4 \mathrm{H}, \mathrm{CH}) ; 3.50\left(\mathrm{br} \mathrm{s}, 2 \mathrm{H}, \mathrm{CH}_{2}\right) ; 1.82\left(\mathrm{br} \mathrm{s}, 6 \mathrm{H}, \mathrm{N}\left(\mathrm{CH}_{3}\right)_{2}\right)$;

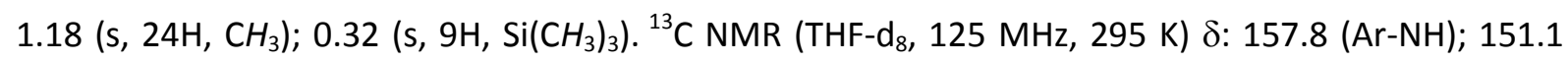
$\left(\mathrm{Ar}^{\mathrm{Dipp}}-\mathrm{N}\right)$; $131.3(\mathrm{Ar}) ; 128.0(\mathrm{Ar}) ; 124.7\left(\mathrm{Ar}-\mathrm{CH}_{2}\right) ; 124.6(\mathrm{Ar}) ; 123.9$ (br s, $\left.\mathrm{Ar}\right) ; 116.2(\mathrm{Ar}) ; 64.0\left(\mathrm{CH}_{2}\right)$; $45.8\left(\mathrm{NCH}_{3}\right) ; 28.8$ (br s, $\left.\mathrm{CH}\right) ; 25.8\left(\mathrm{br} \mathrm{s}, \mathrm{CH}_{3}\right) ; 24.2$ (br s, $\left.\mathrm{CH}_{3}\right) 3.5\left(\mathrm{Si}\left(\mathrm{CH}_{3}\right)_{3}\right)$; rest of signals remained hidden due to fast exchange processes of some fragment in the molecule. ${ }^{7} \mathrm{Li}$ NMR $\left(T H F-d_{8}, 194 \mathrm{MHz}\right.$, $295 \mathrm{~K}) \delta: 1.8$.

\section{References}

1 Patton, J. T.; Bokota, M. M.; Abboud, K. A. Indium-Bridged Chelating Diamide Group IV Metal Olefin Polymerization Catalysts. Organometallics 2002, 21, 2145-2148.

2 Luo, B.; Young, V. G.; Gladfelter, W. L. Si-C bond cleavage in the reaction of gallium chloride with lithium bis(trimethylsilyl)amide and thermolysis of base adducts of dichloro(trimethylsilyl)amido gallium compounds. J. Organomet. Chem. 2002, 649, 268-275.

3 Vaňkátová, H.; Broeckaert, L.; De Proft, F.; Olejník, R.; Turek, J.; Padělková, Z.; Růžička, A. Tetrylenes Chelated by Hybrid Amido-Amino Ligand: Derivatives of 2-[(,$N$-Dimethylamino)methyl]aniline Inorg. Chem. 2011, 50, 9454-9464.

4 Patt, S.; Shoolery, J. N. Attached proton test for carbon-13 NMR J. Magn. Reson. 1982, 46, 535-539. 5 Otwinowski, Z.; Minor, W. Processing of X-ray diffraction data collected in oscillation mode.

Methods Enzymol 1997, 276, 307-326. 
6 Coppens, P. In: Ahmed, F. R.; Hall, S. R.; Huber, C. P. Editors, Crystallographic Computing, 1970, pp. 255 - 270, Copenhagen, Munksgaard.

7 Altomare, A.; Cascarano, G.; Giacovazzo, C.; Guagliardi, A. Early finding of preferred orientation: a new method. J. Appl. Cryst. 1994, 27, 1045-1050.

8 Sheldrick, G. M. SHELXL-97, University of Göttingen: Göttingen, 2008 


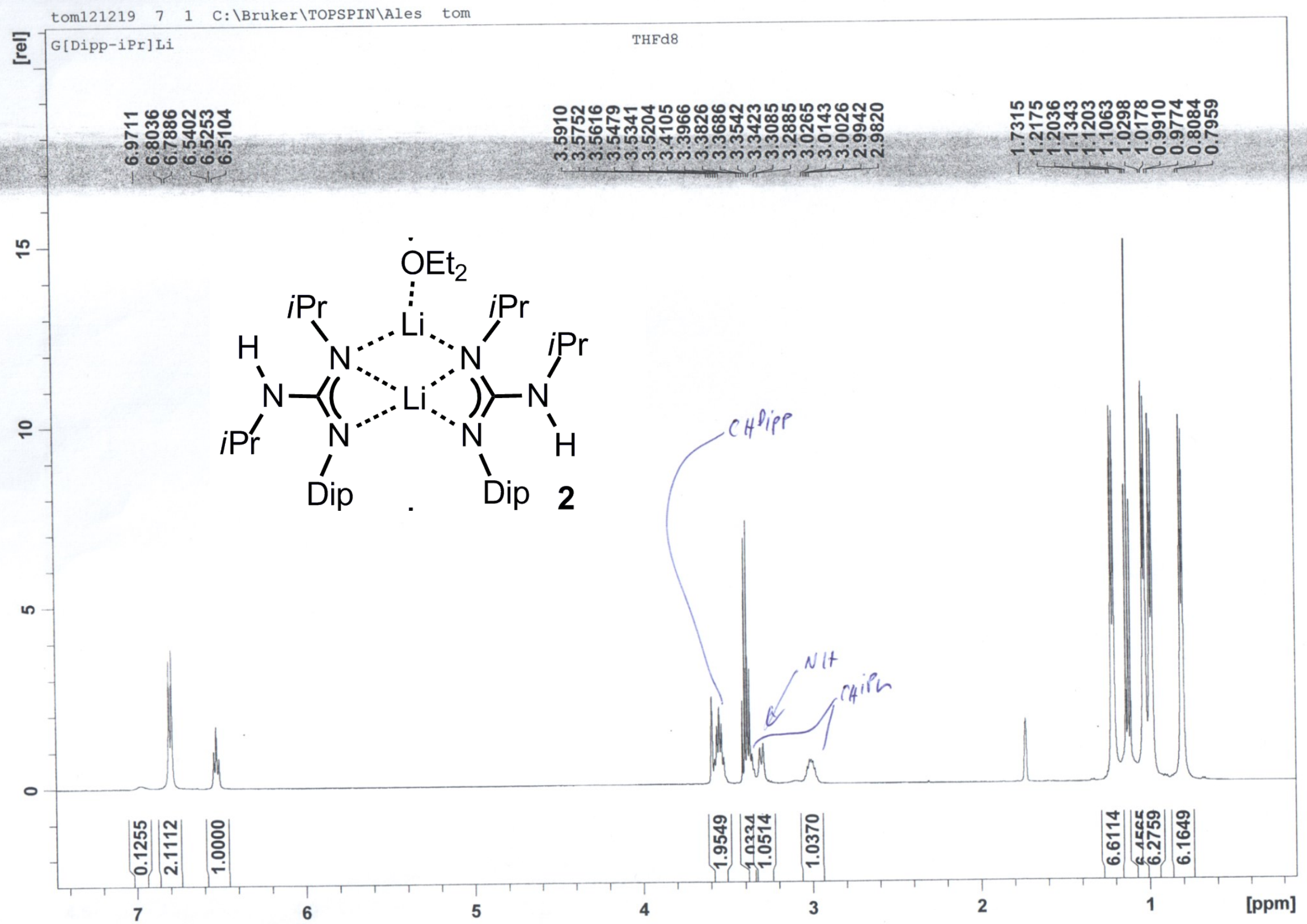




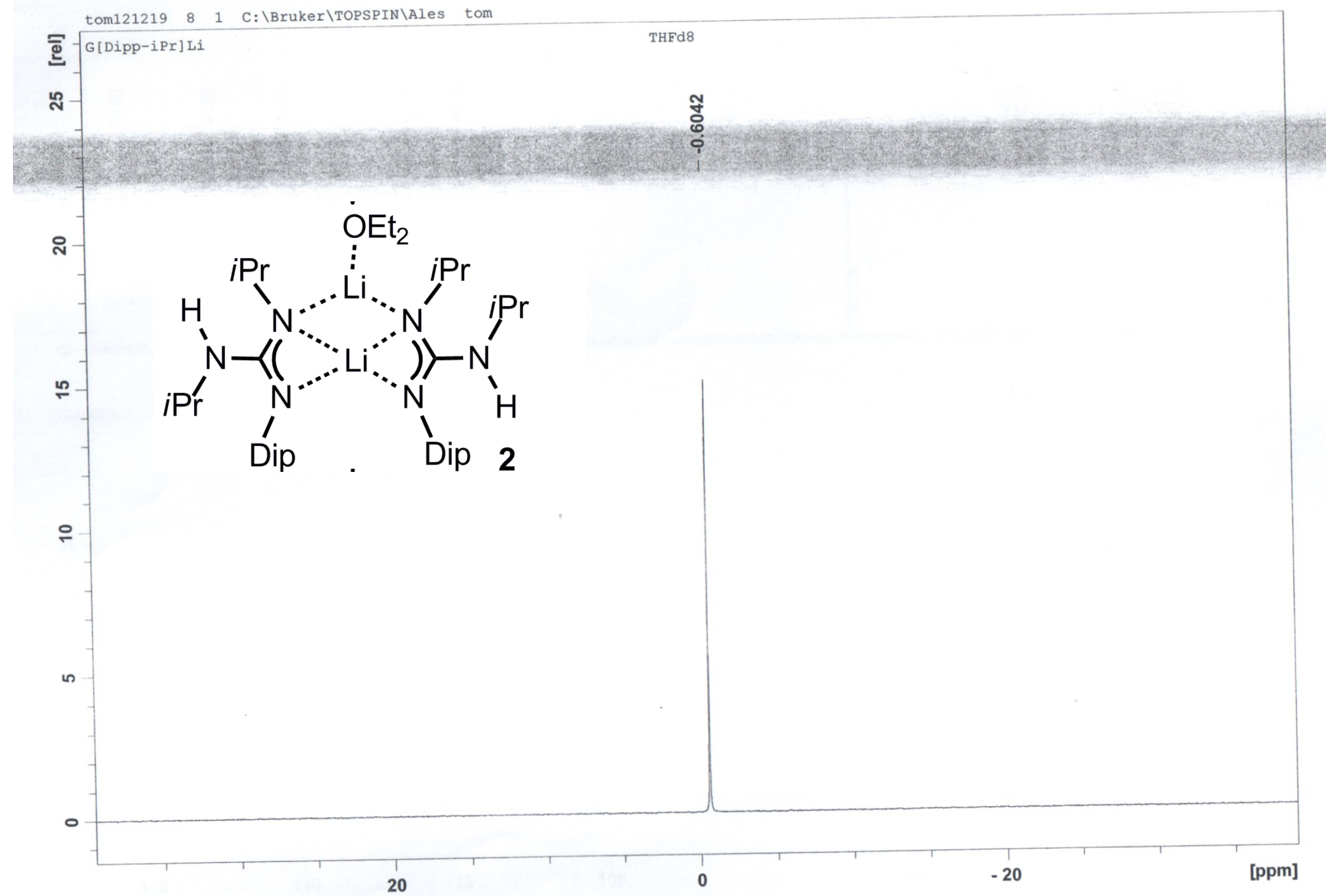




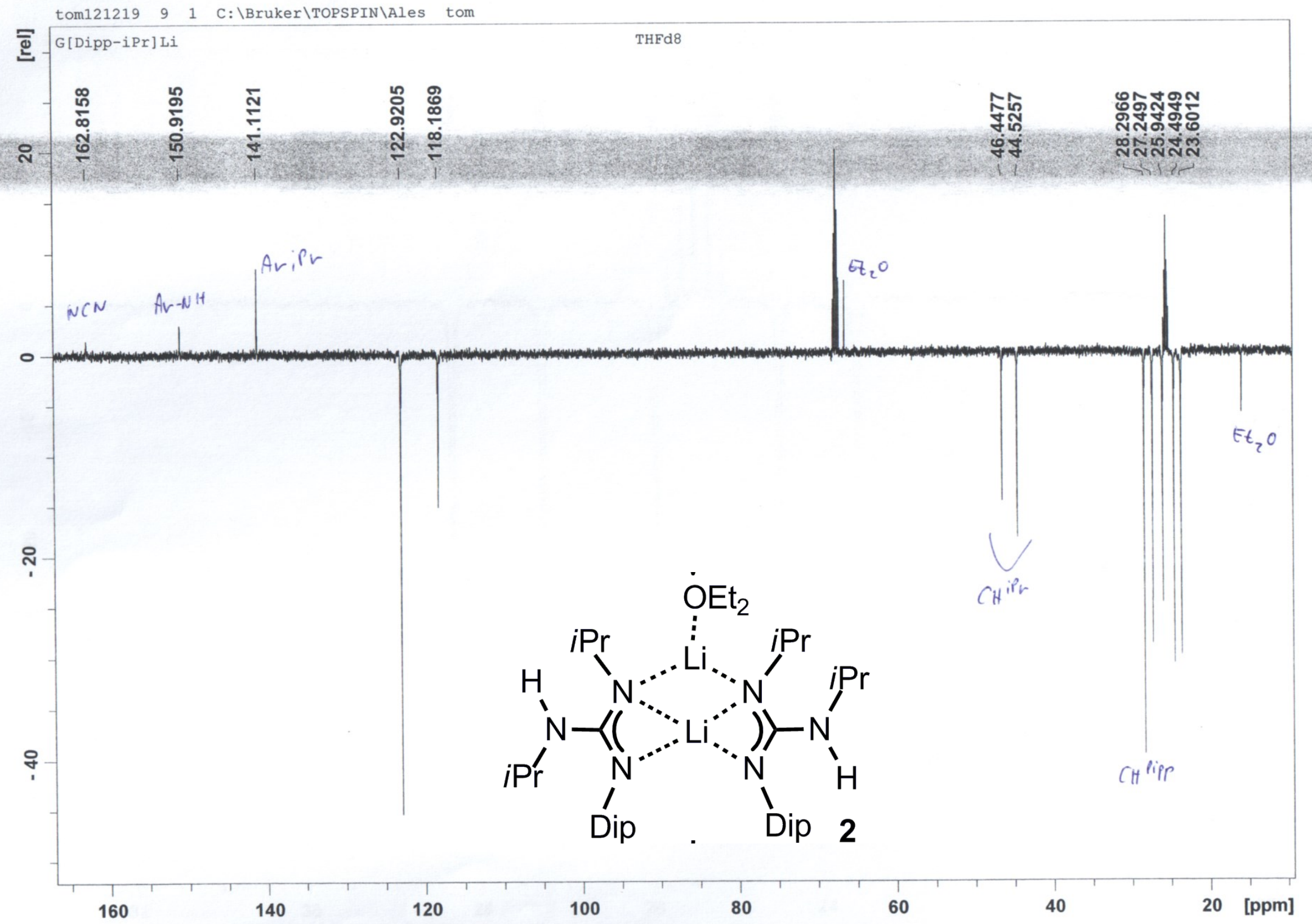




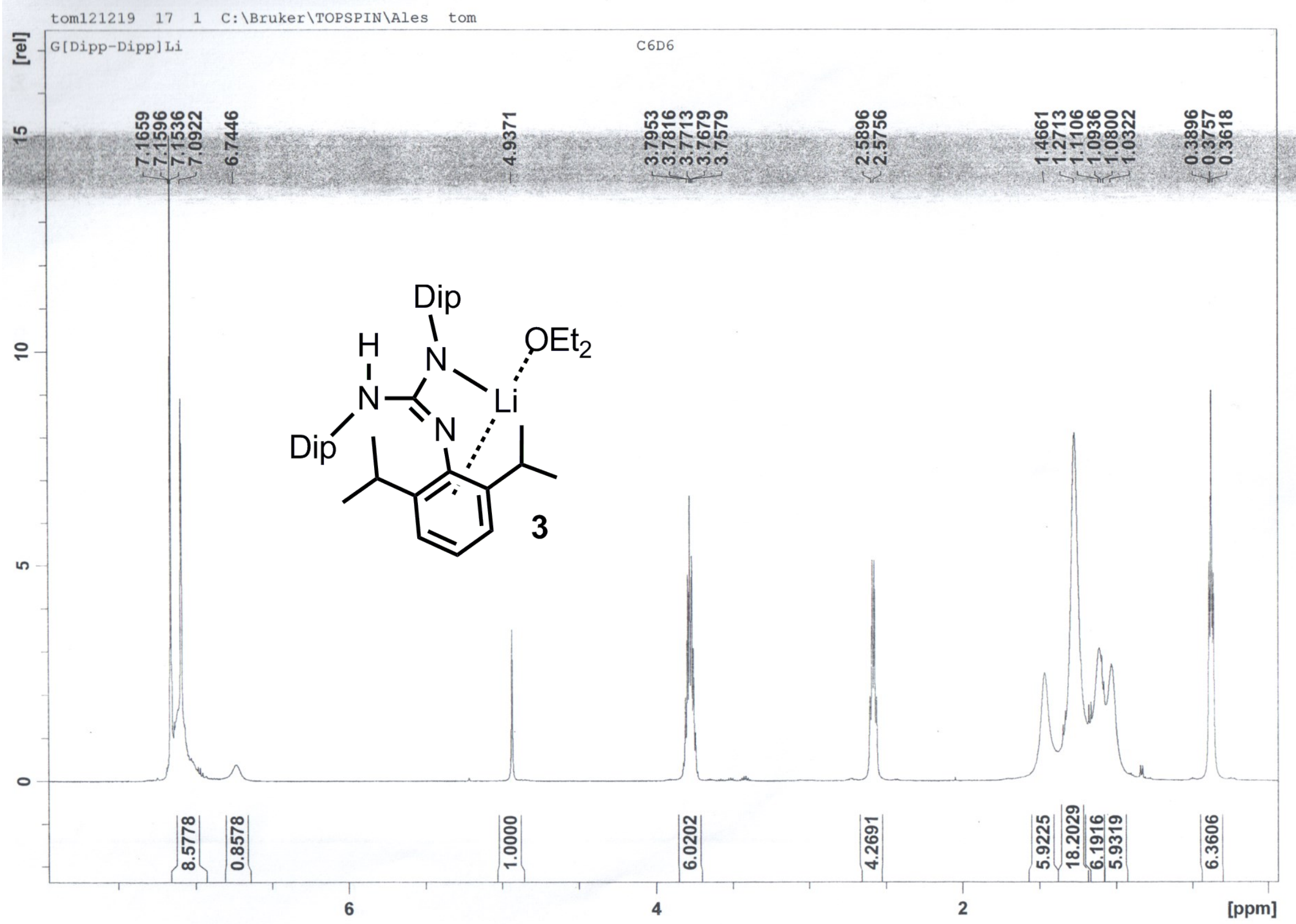




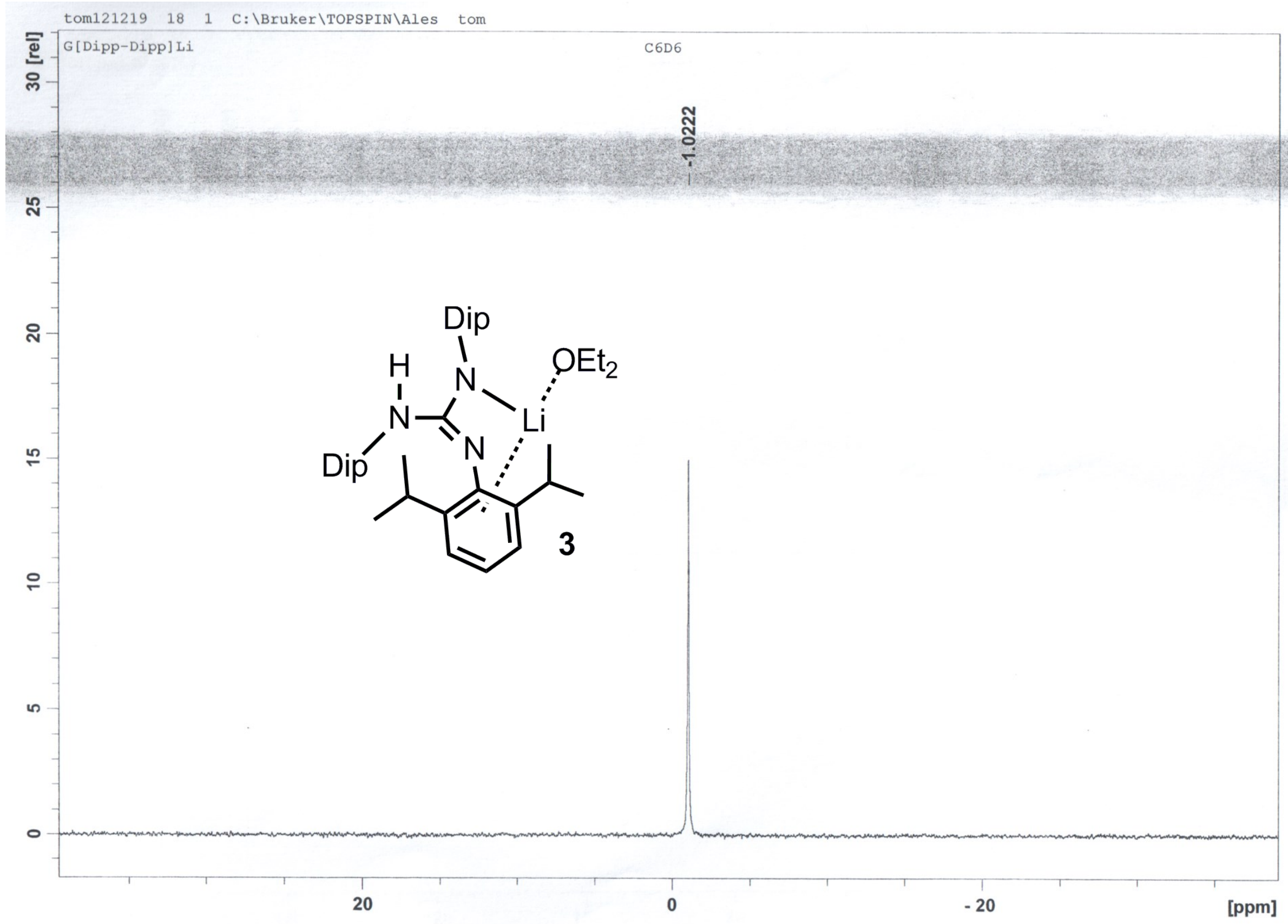




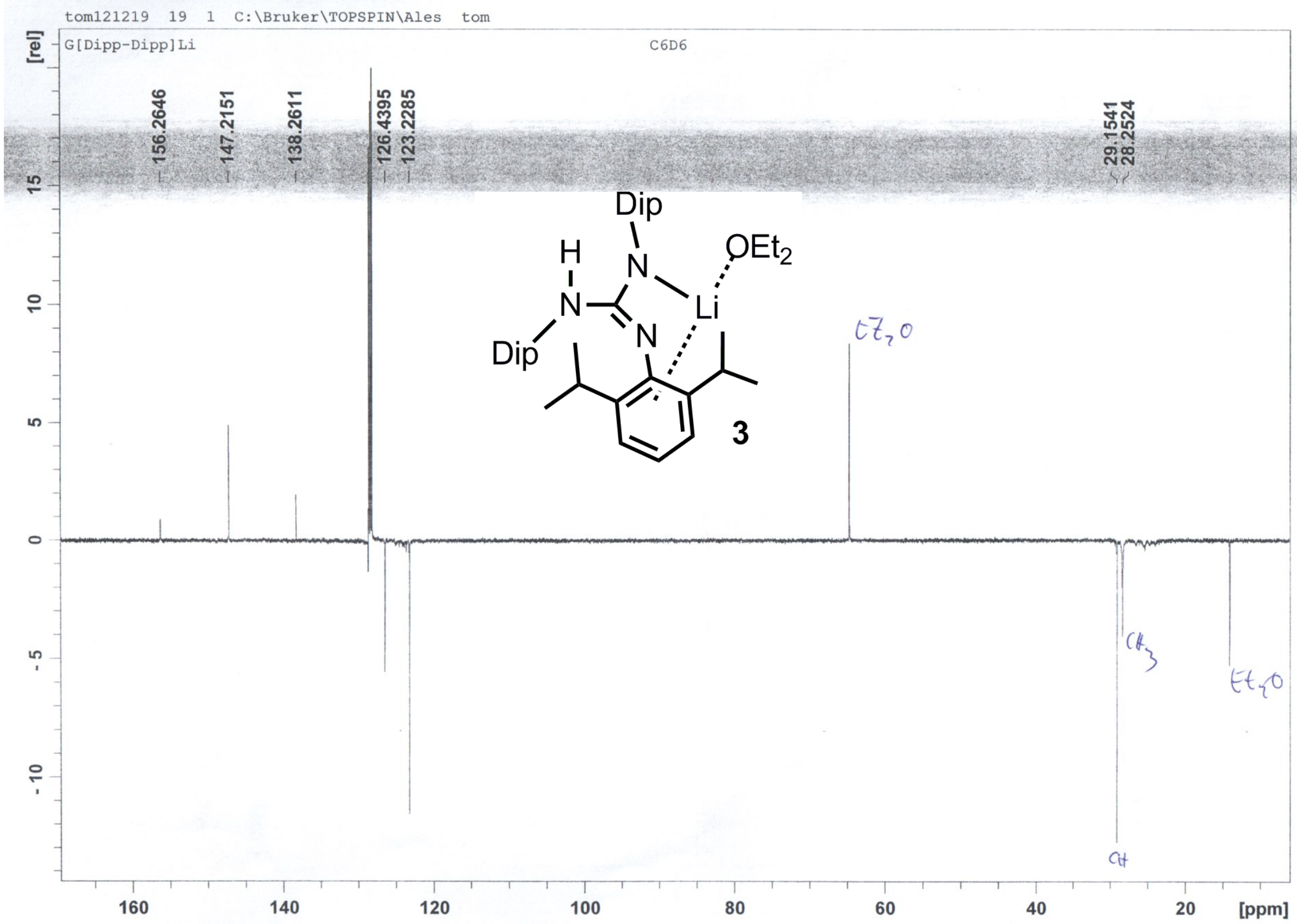




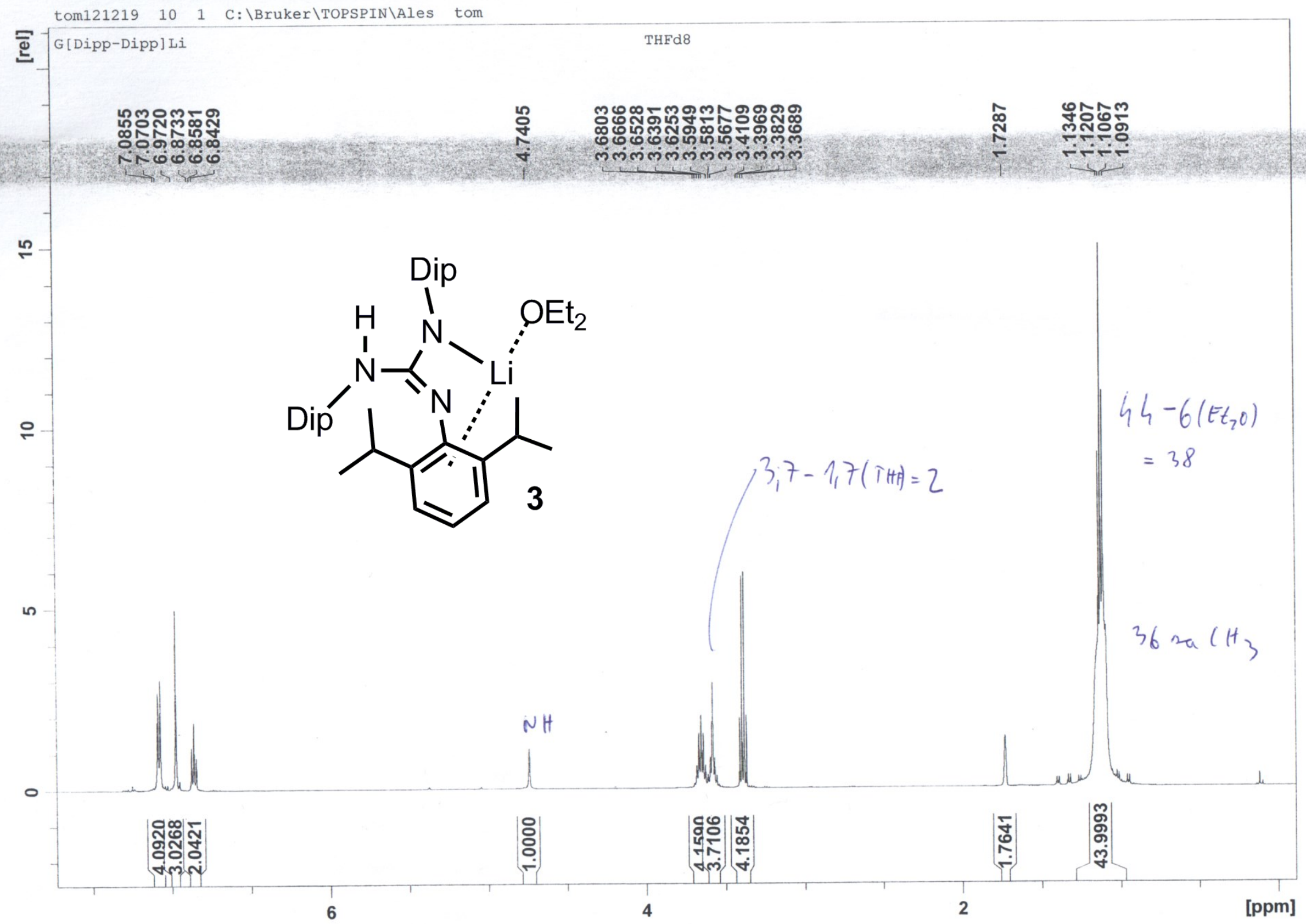




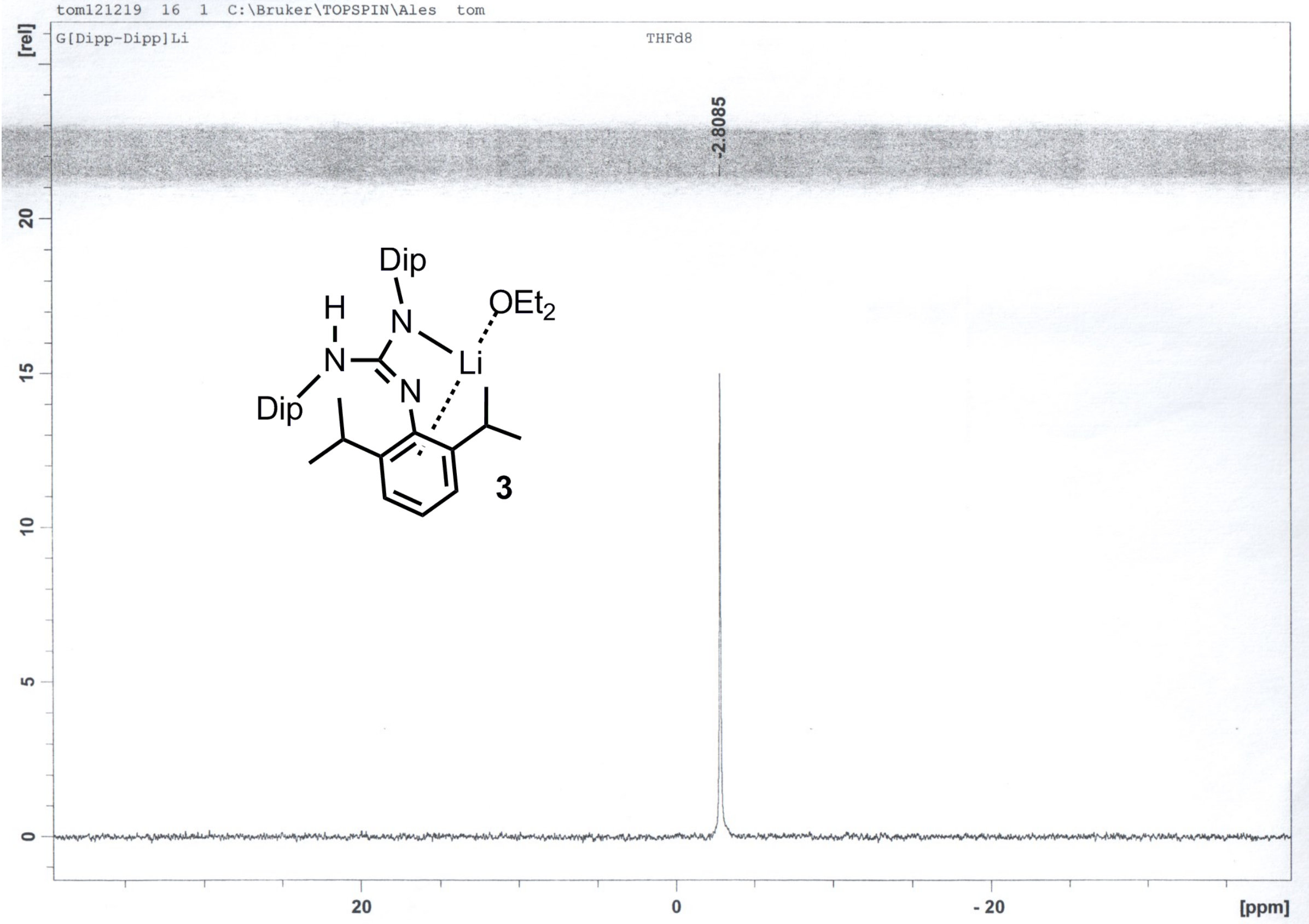




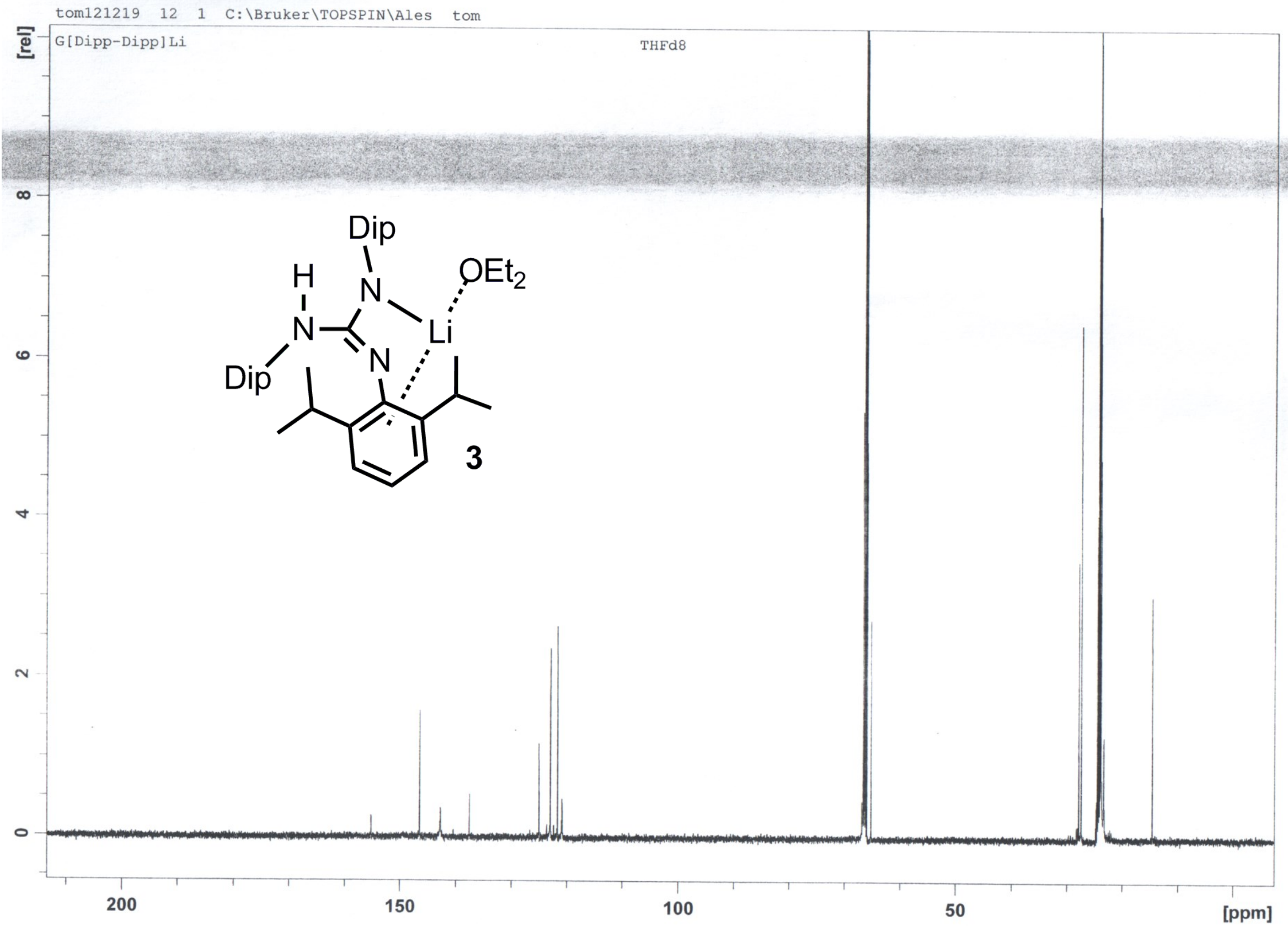




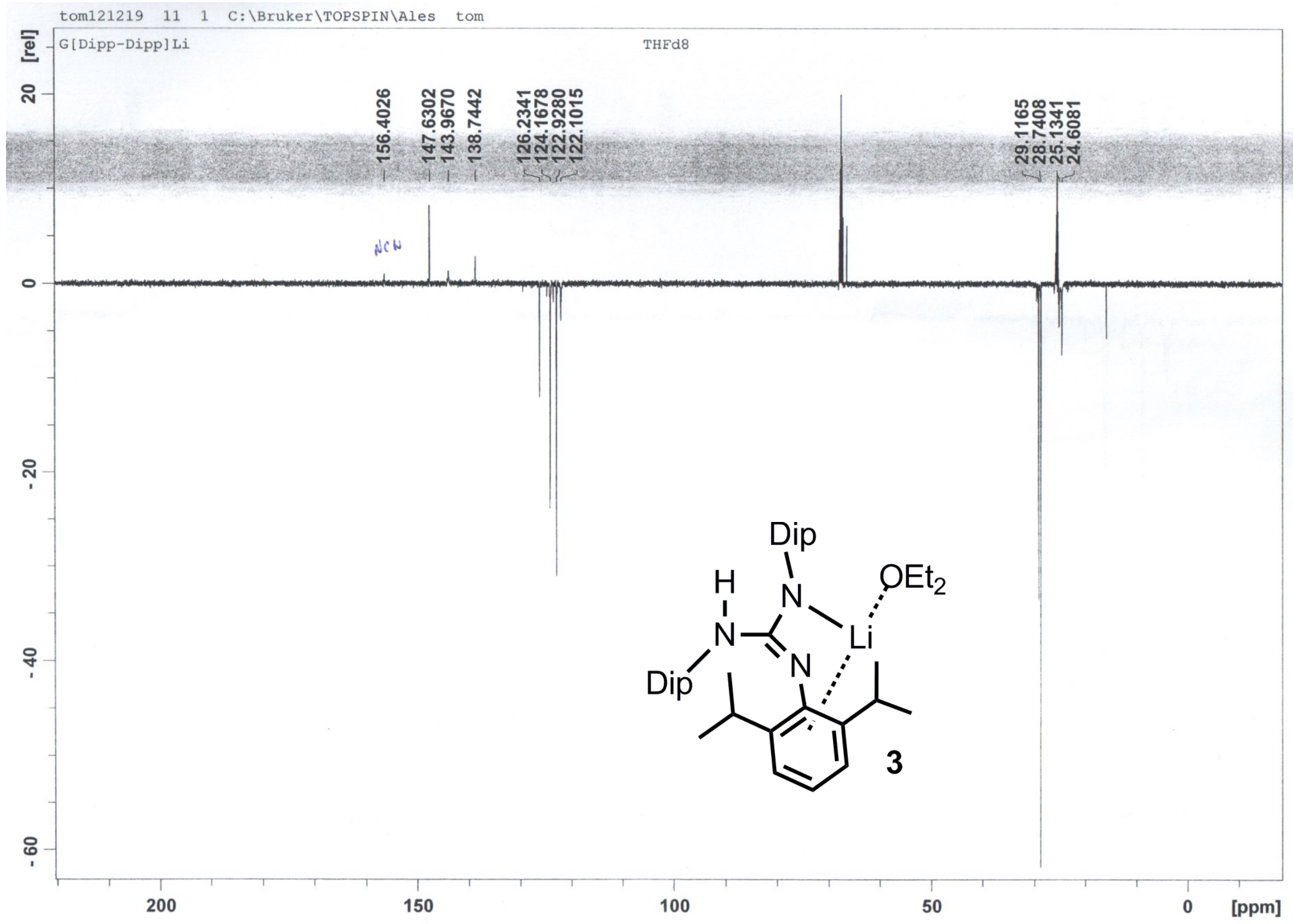




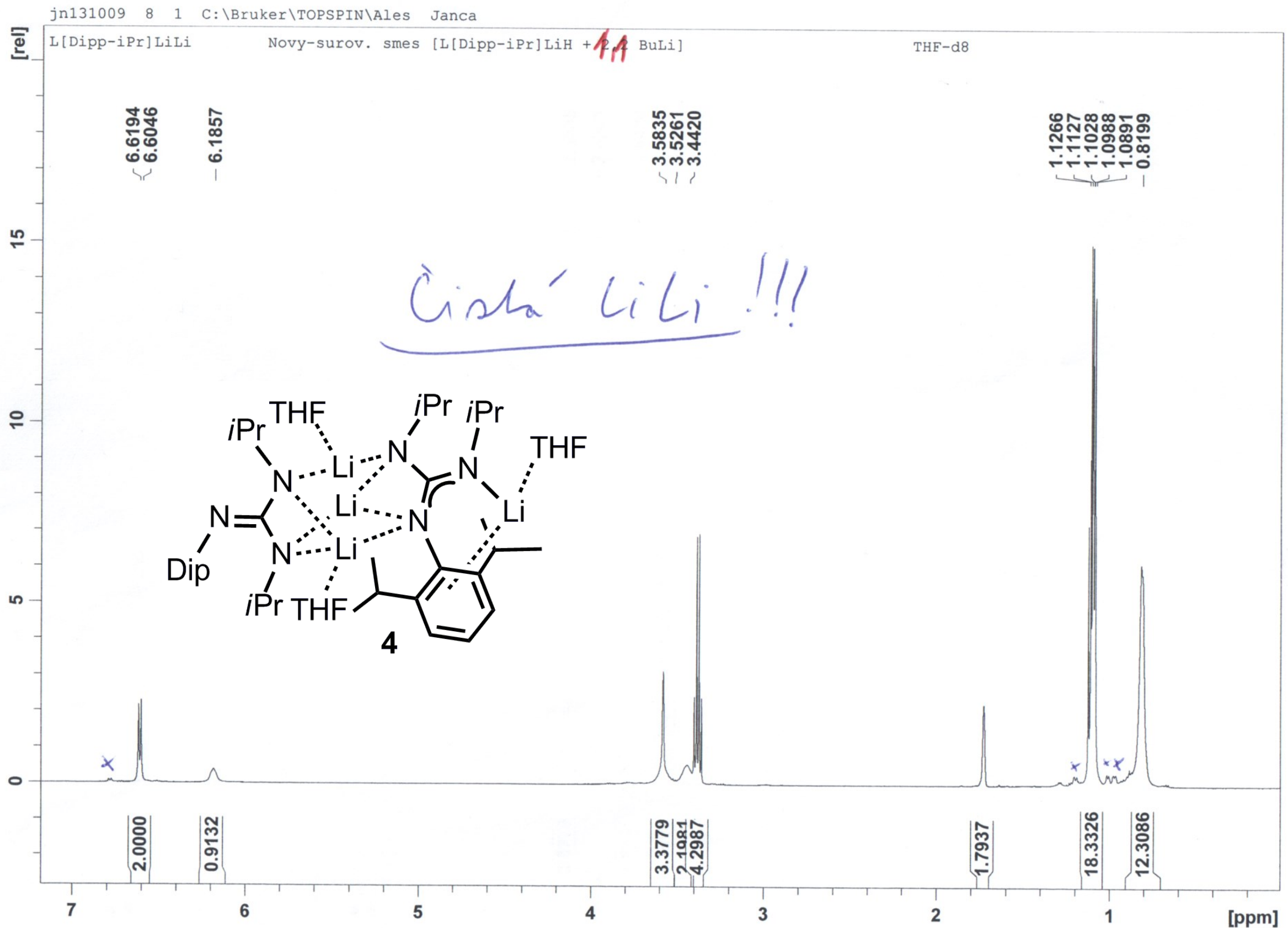




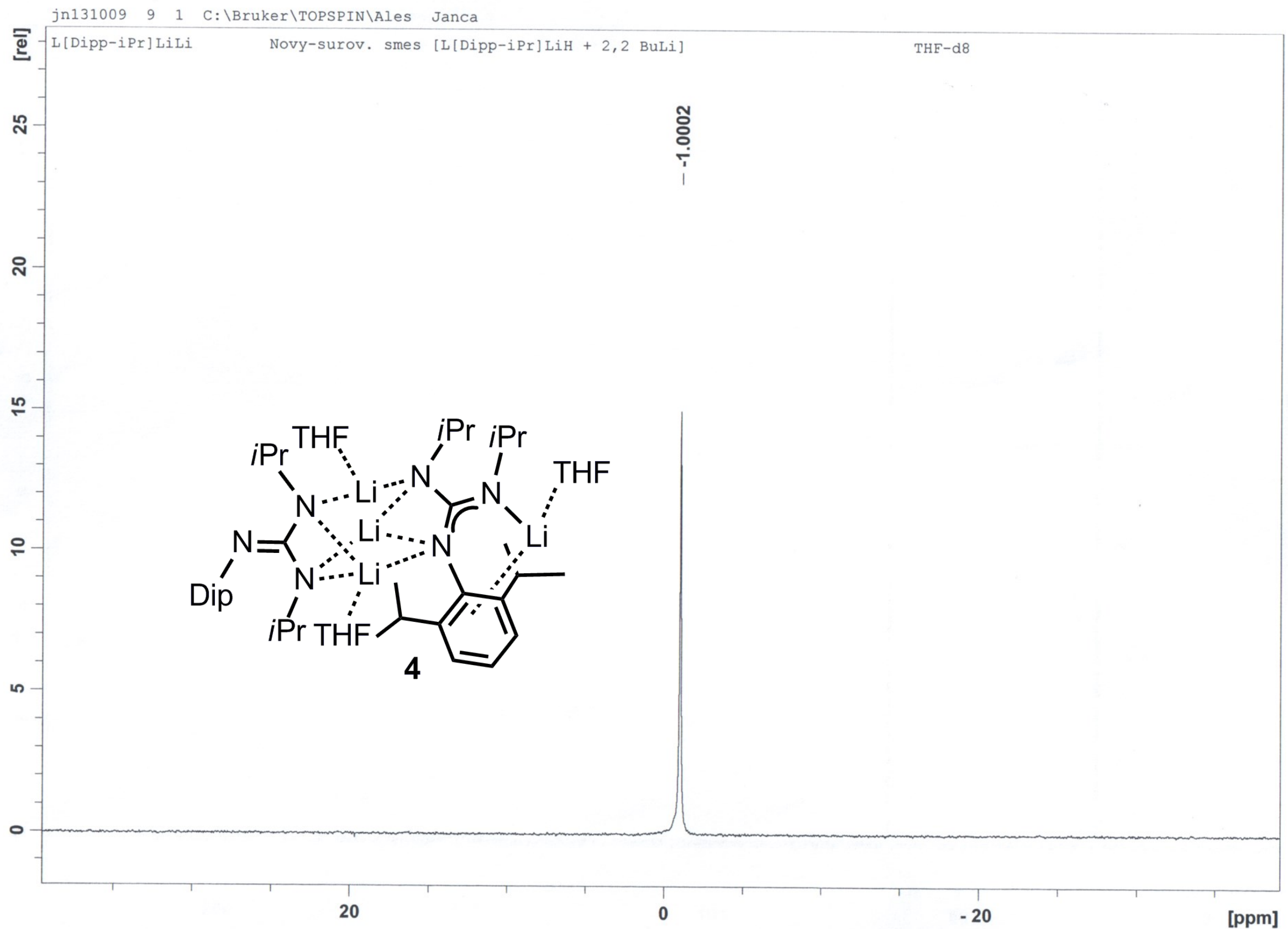




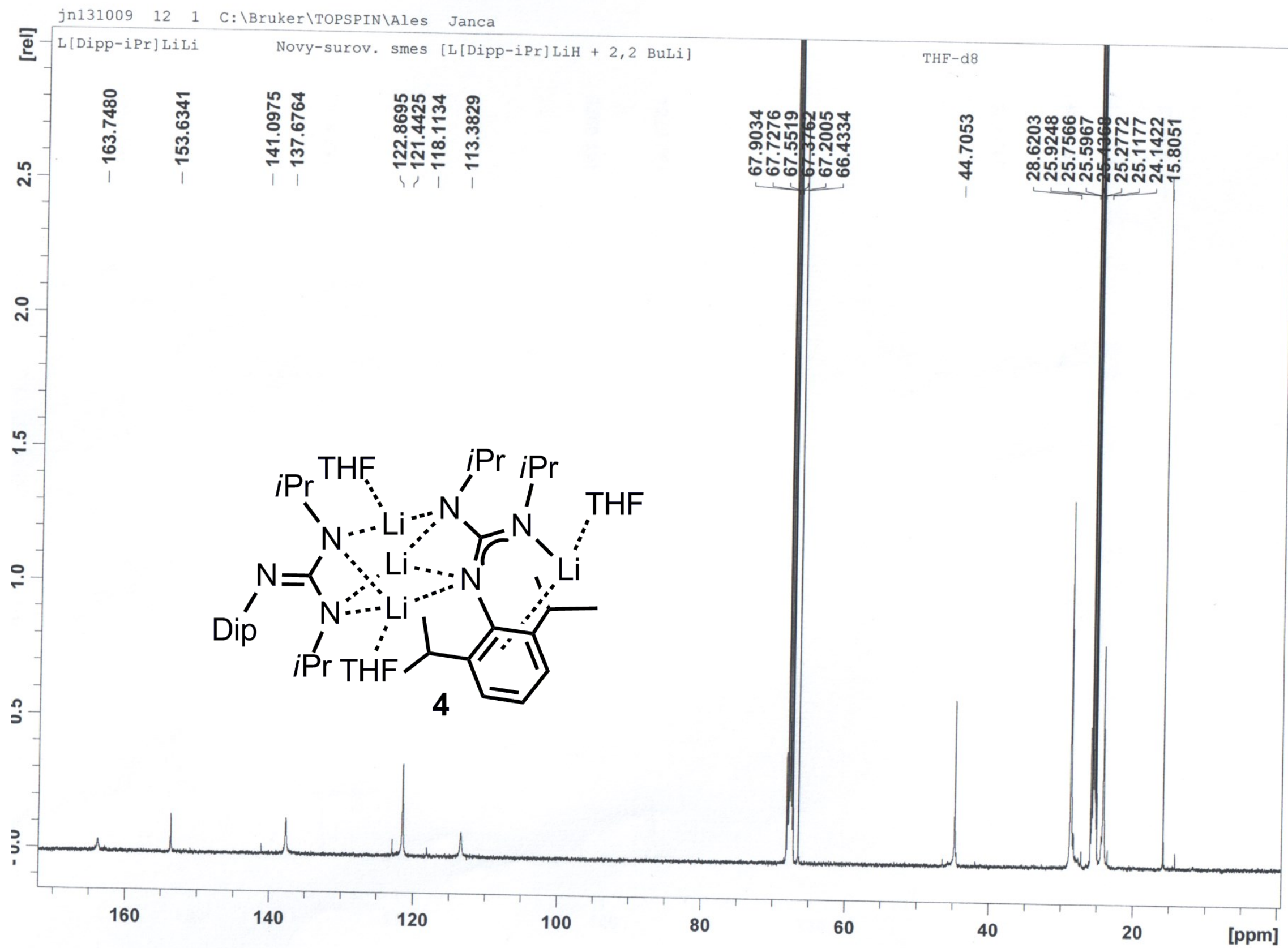




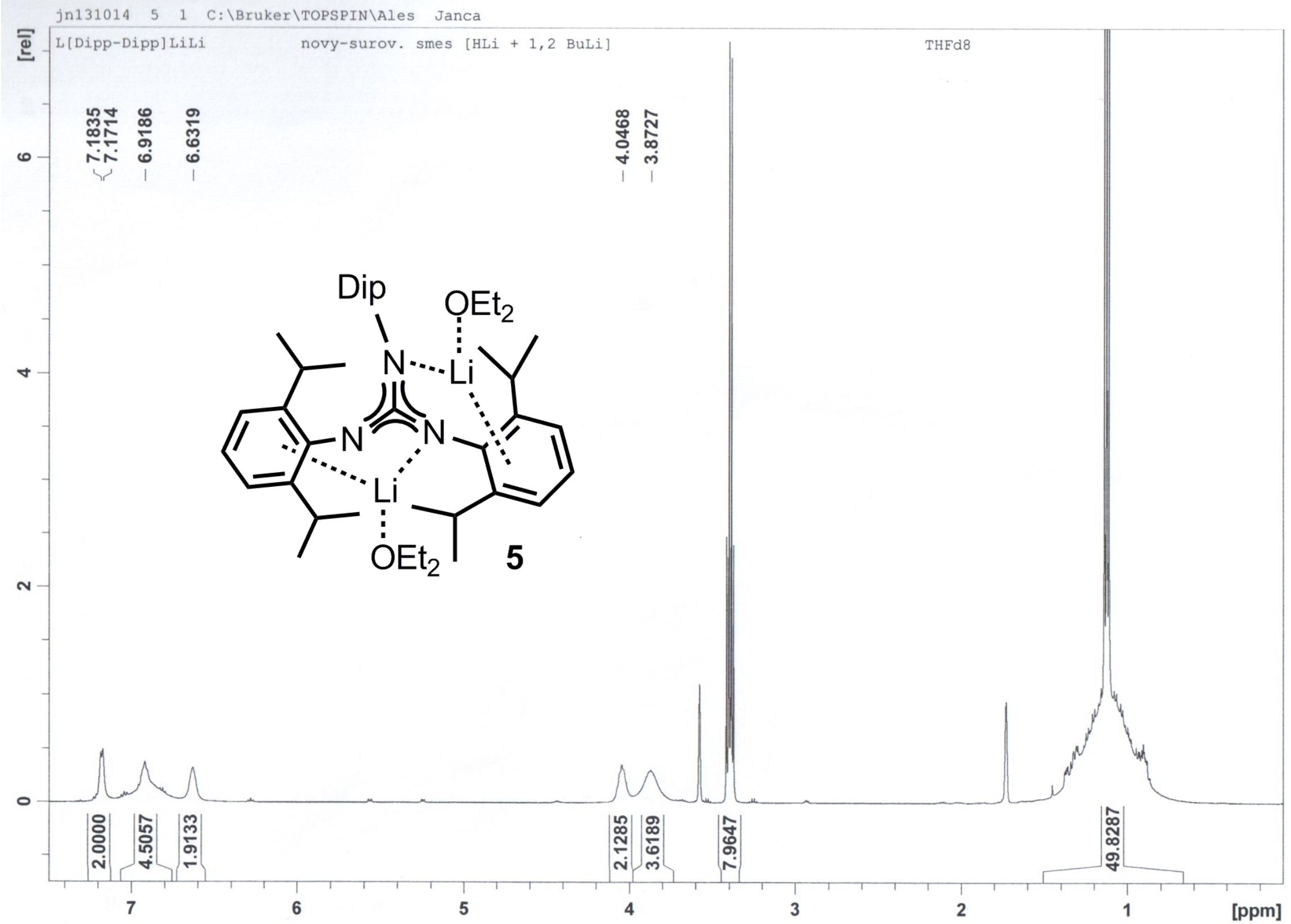




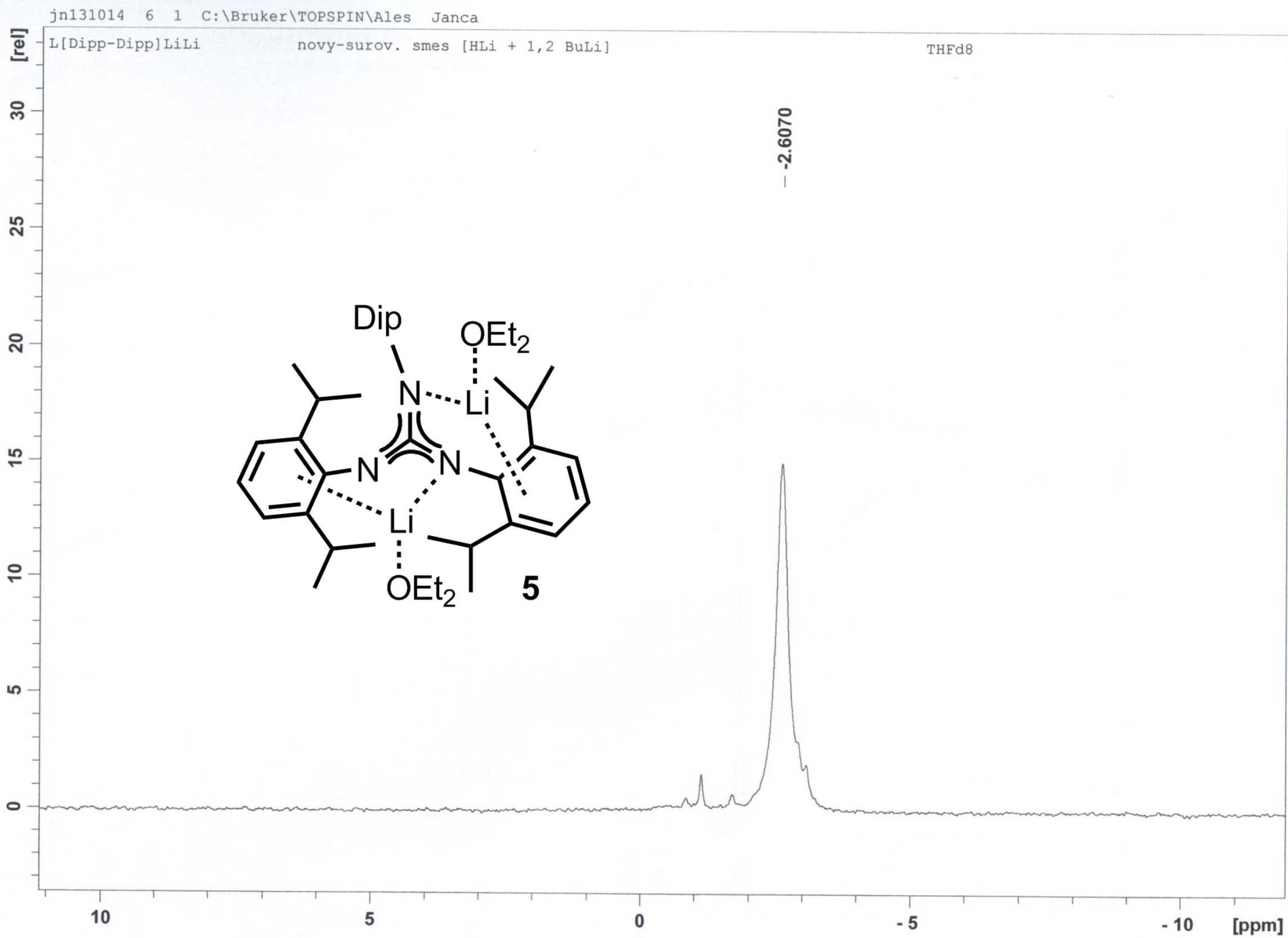




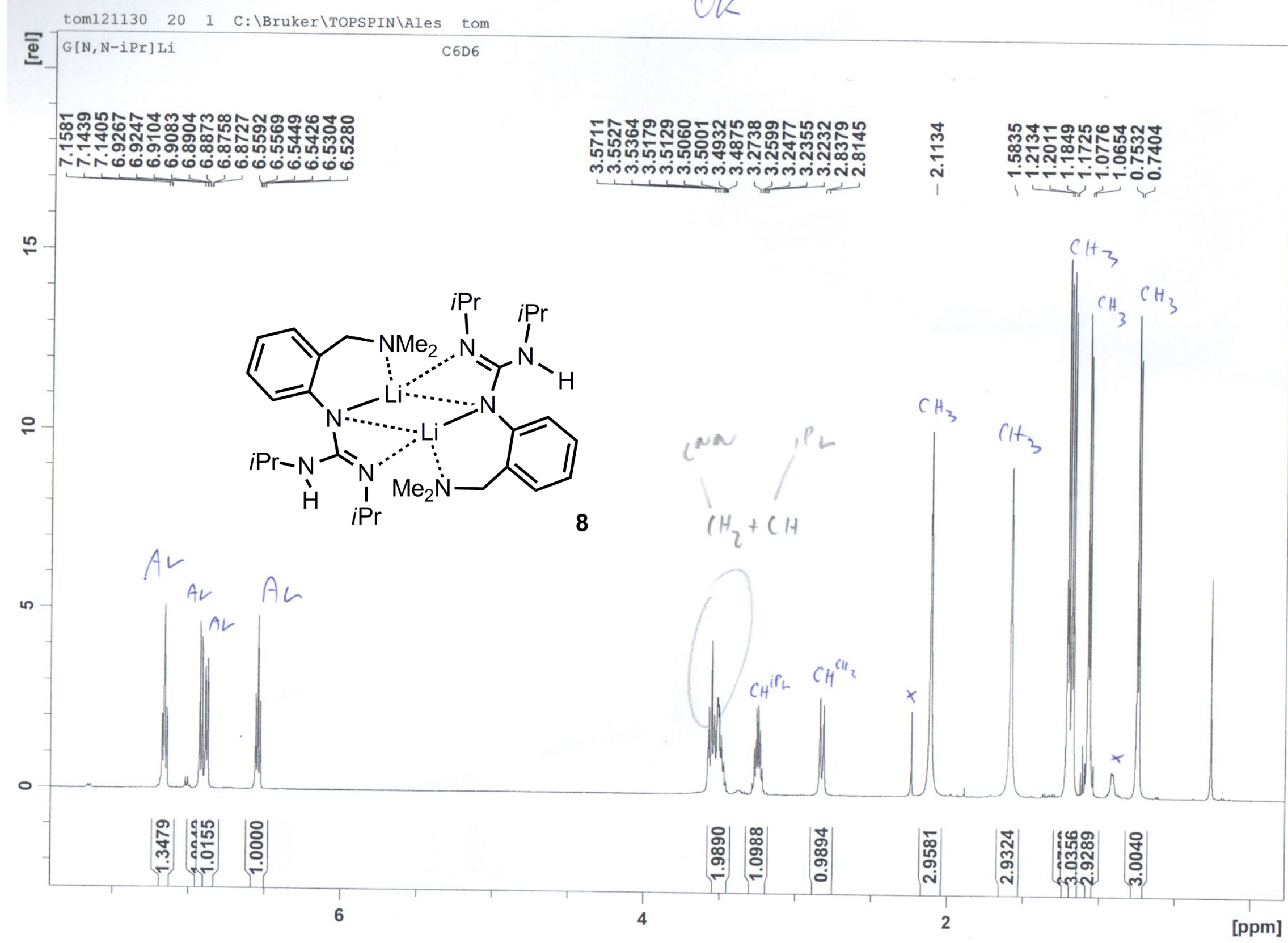




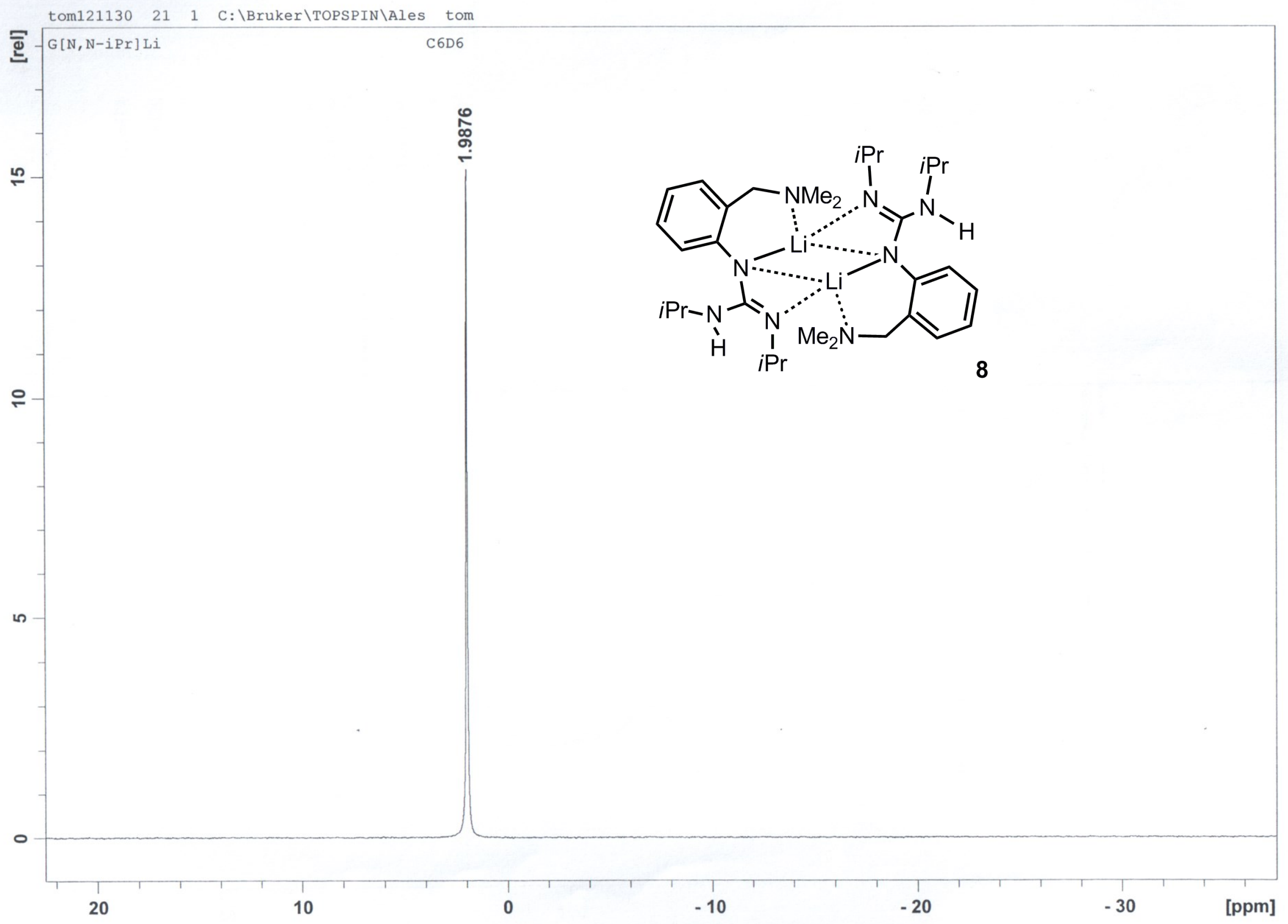




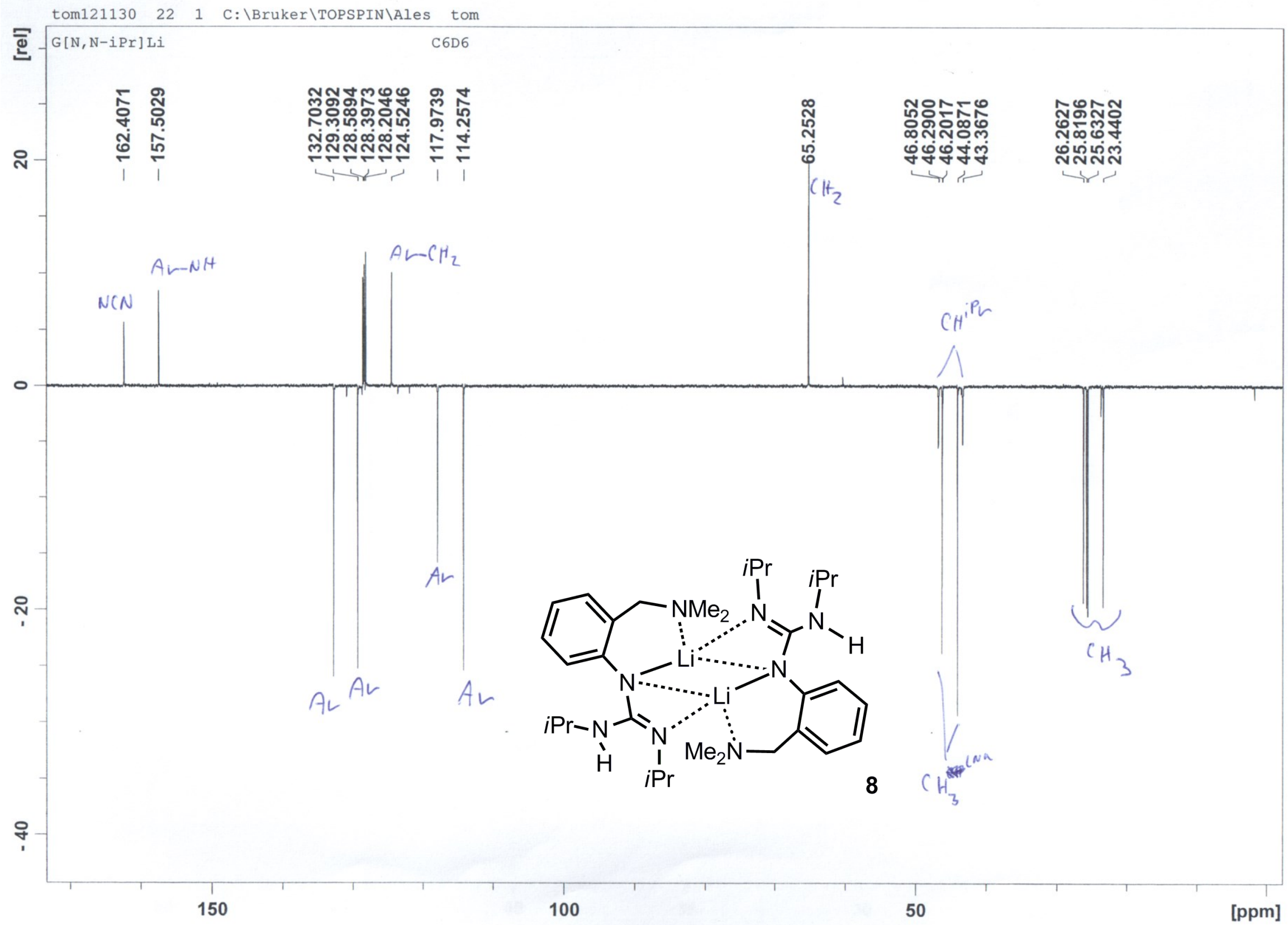




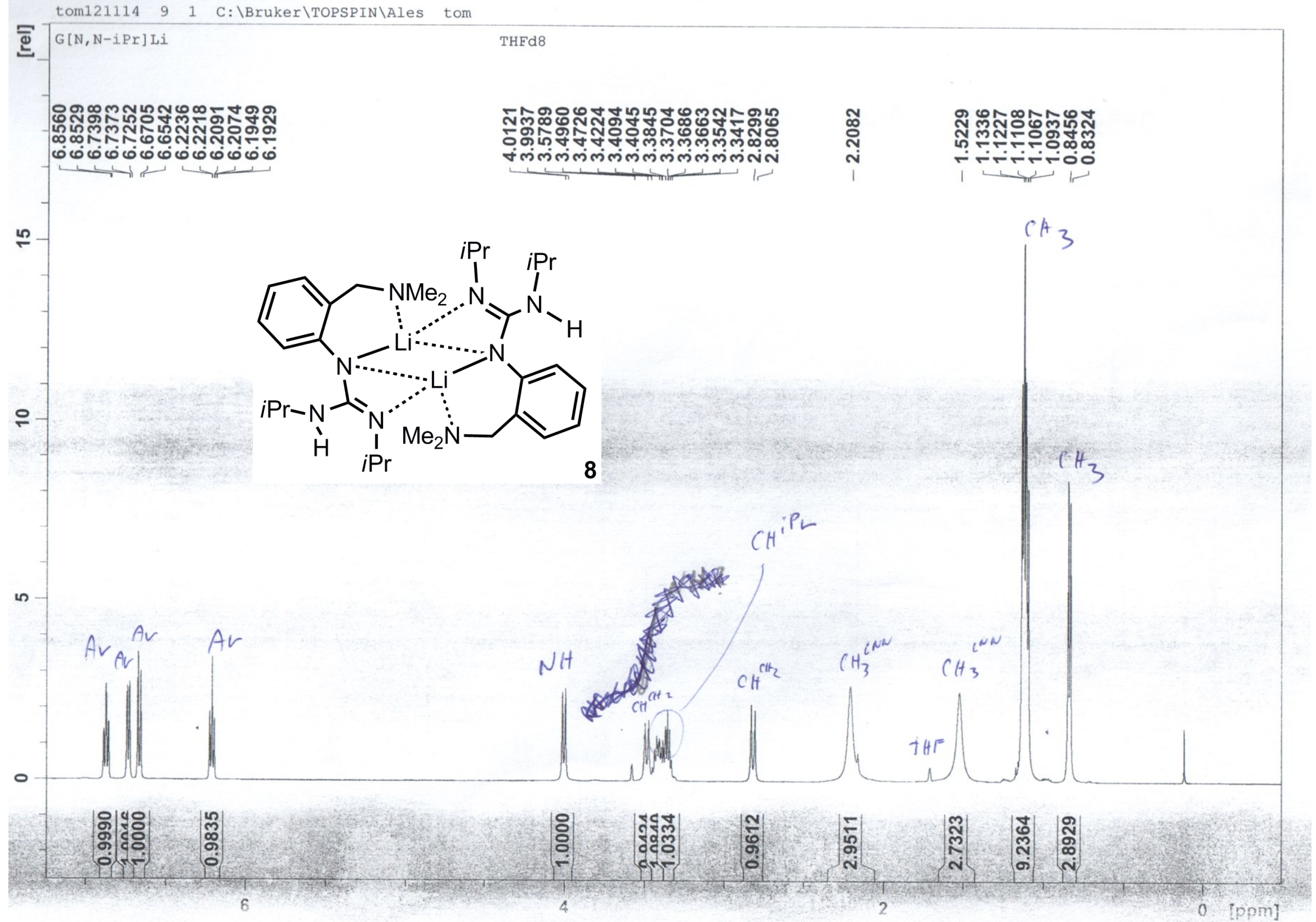




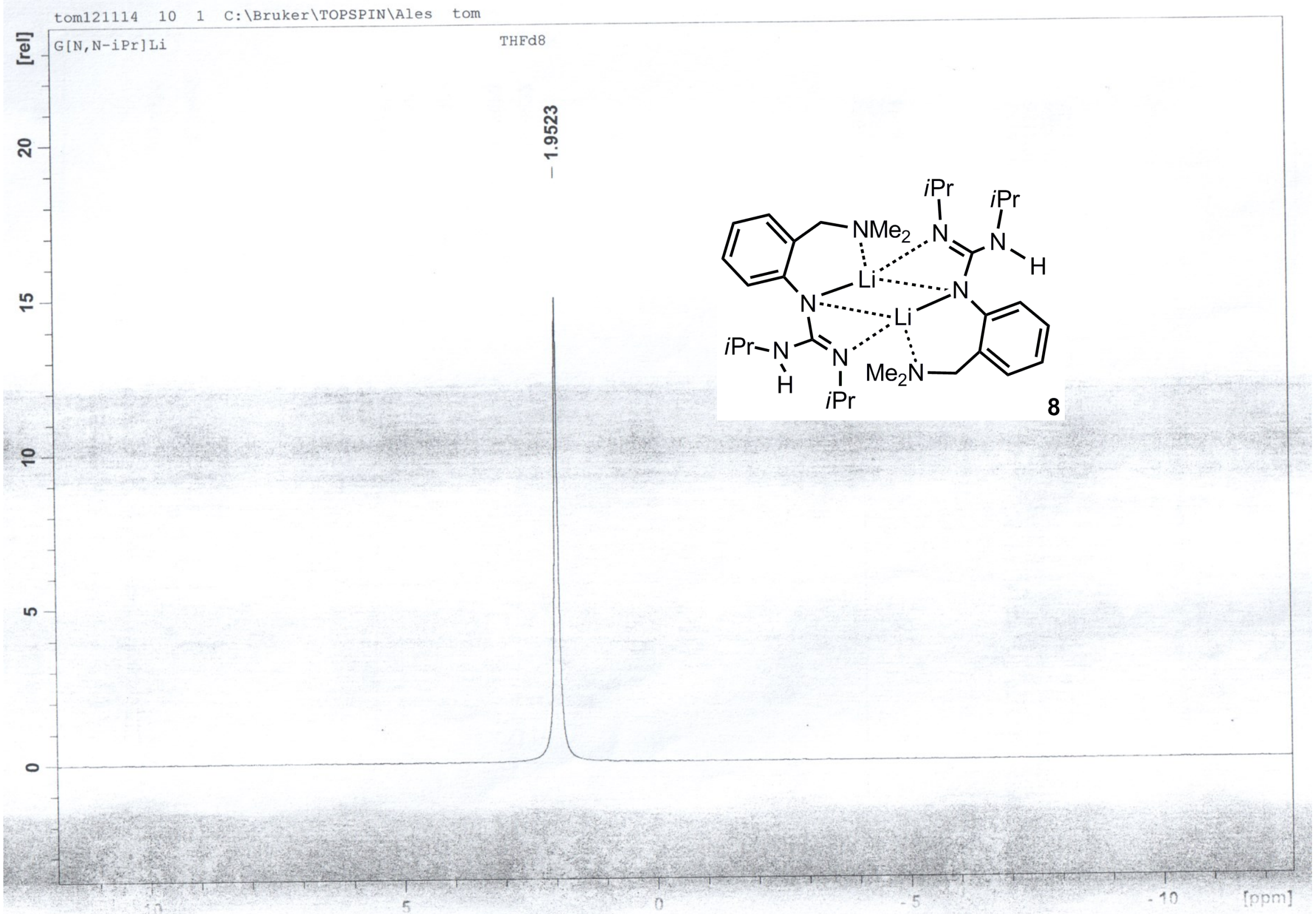




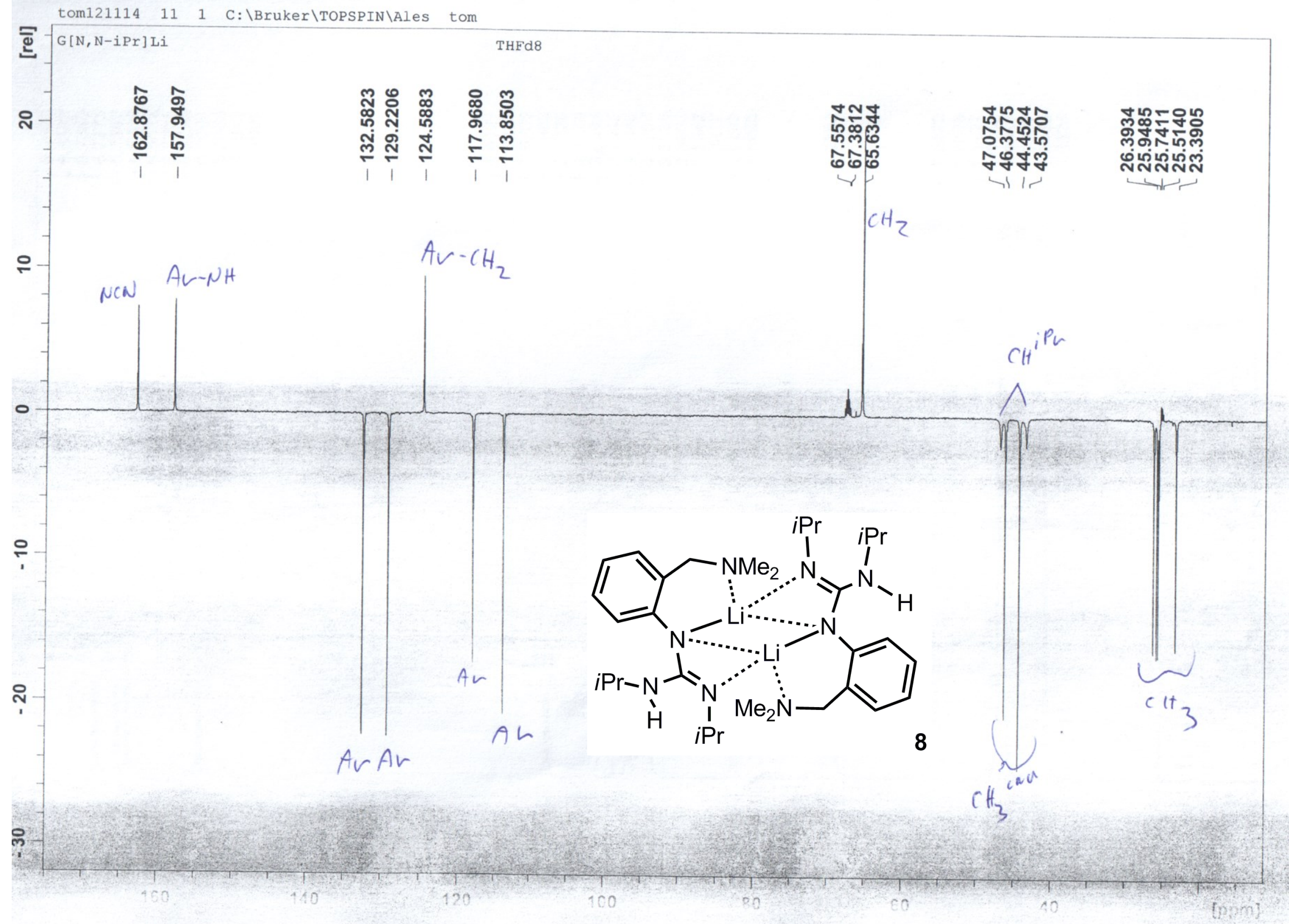




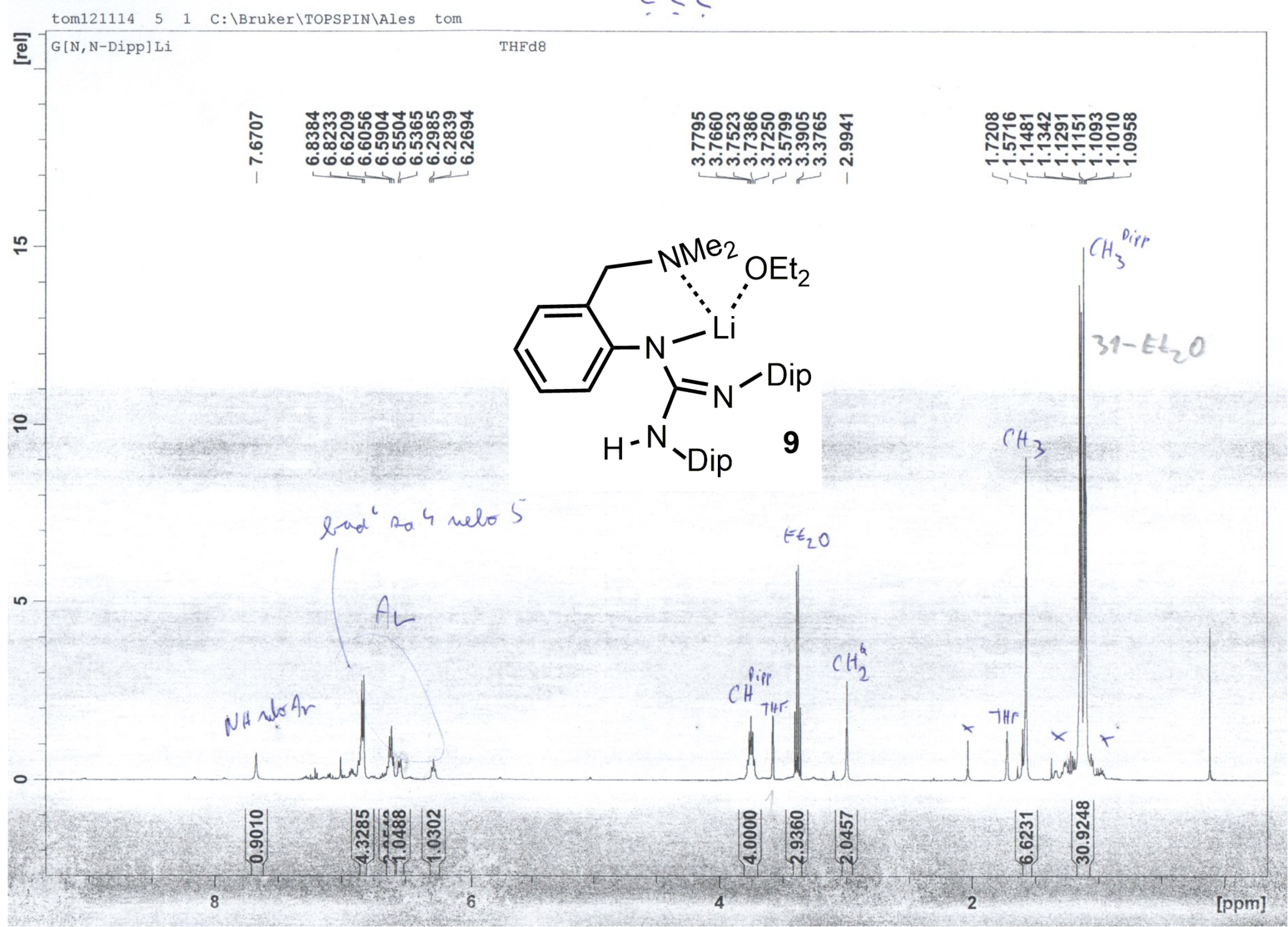




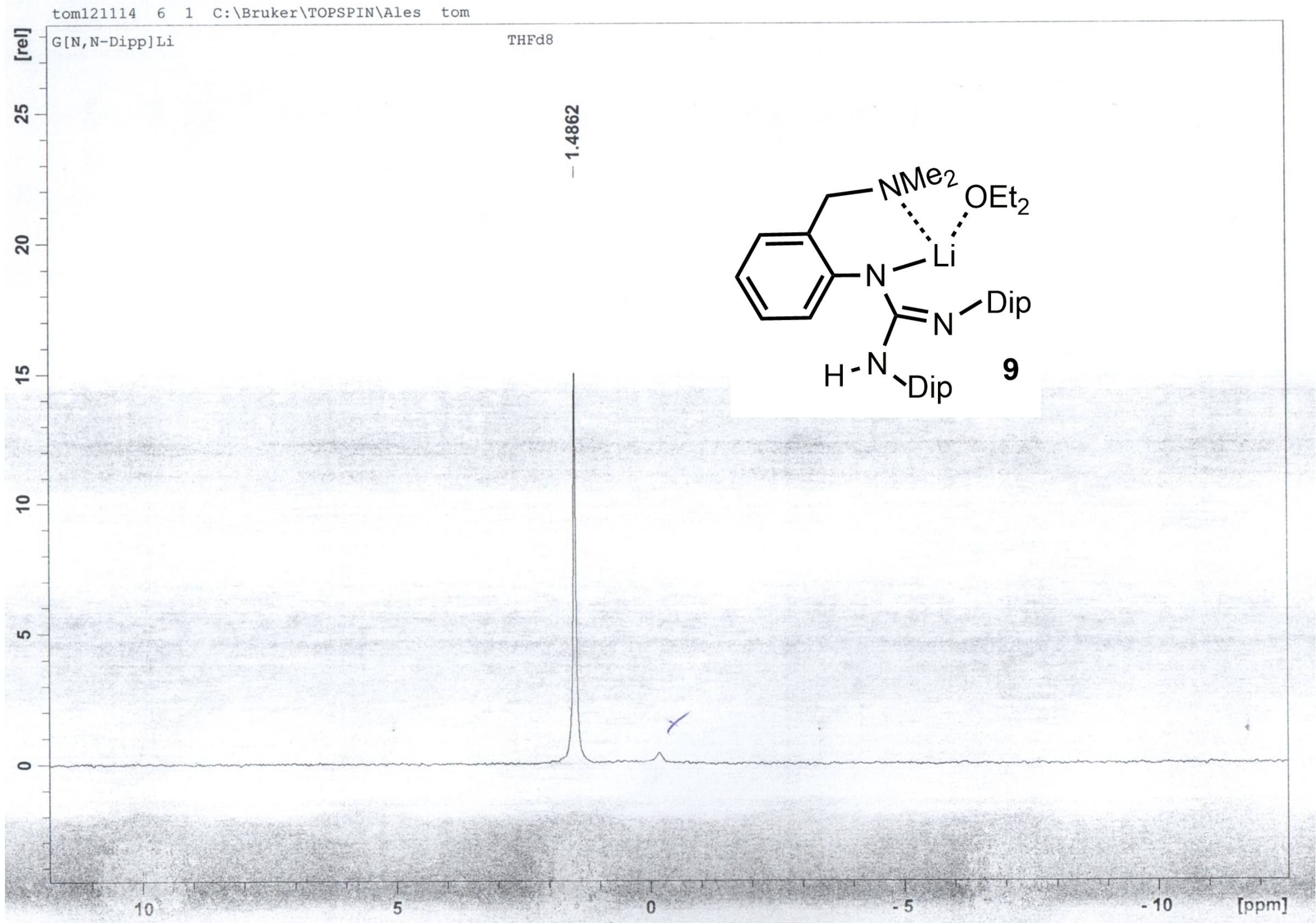




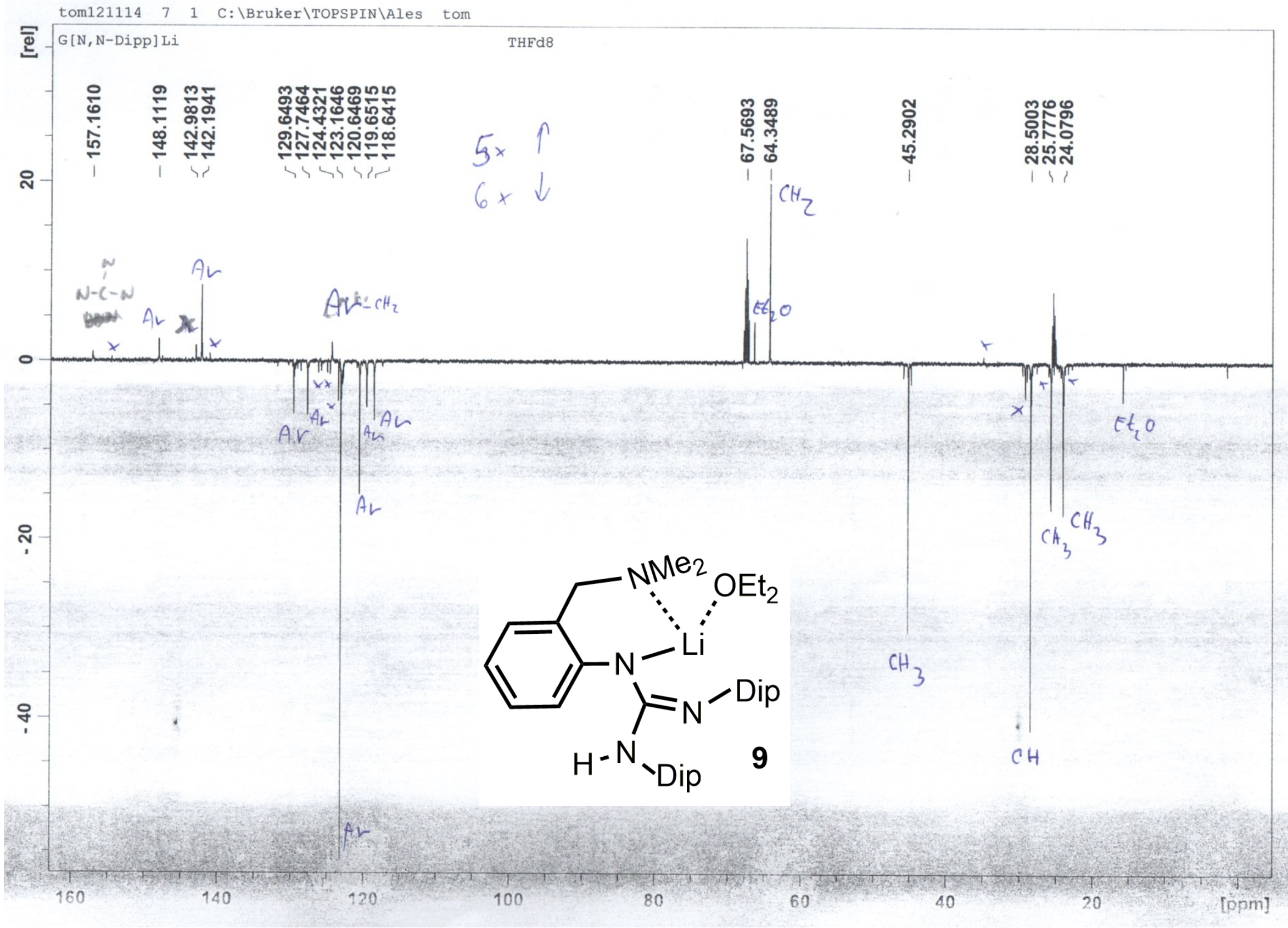




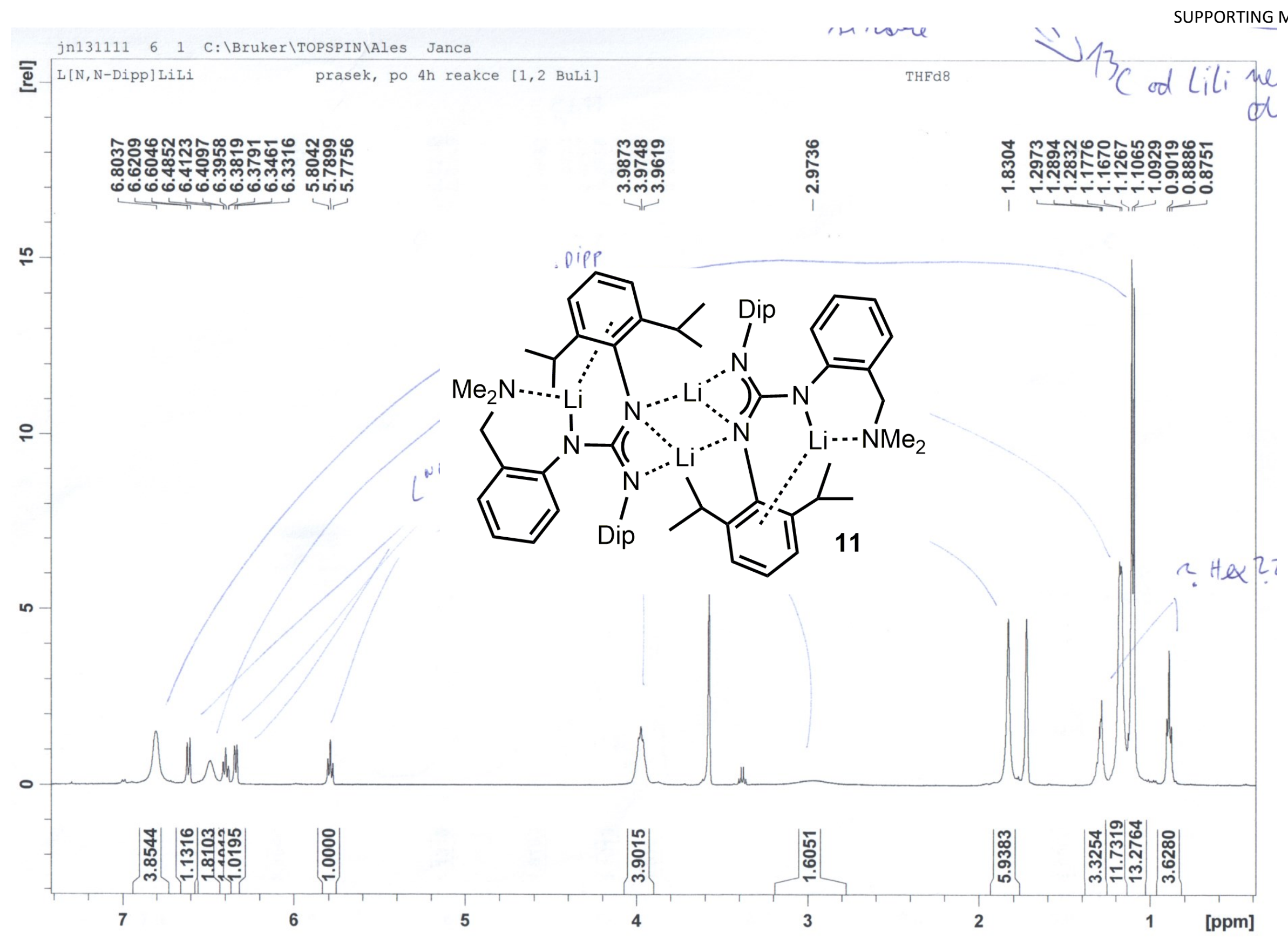




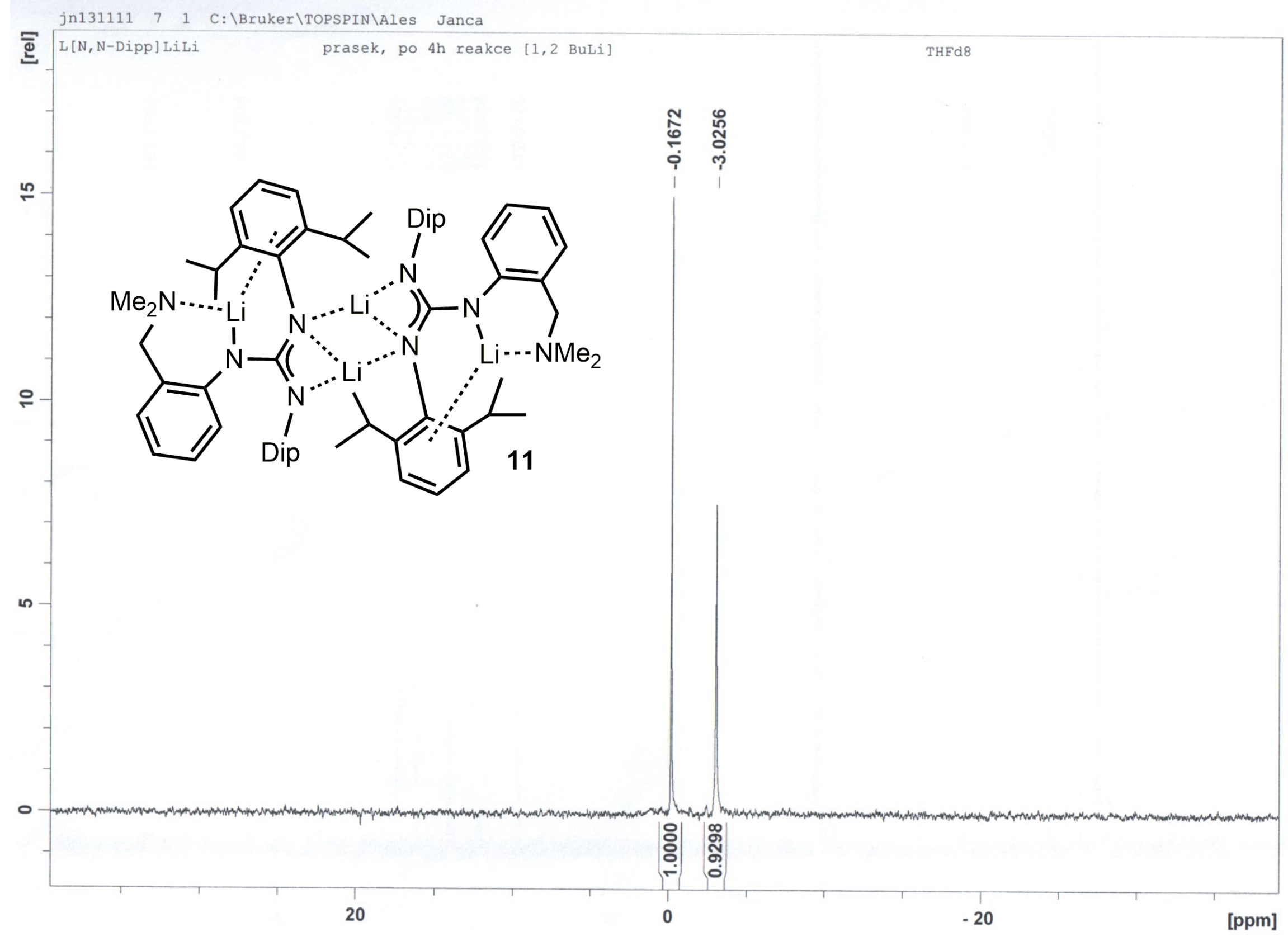




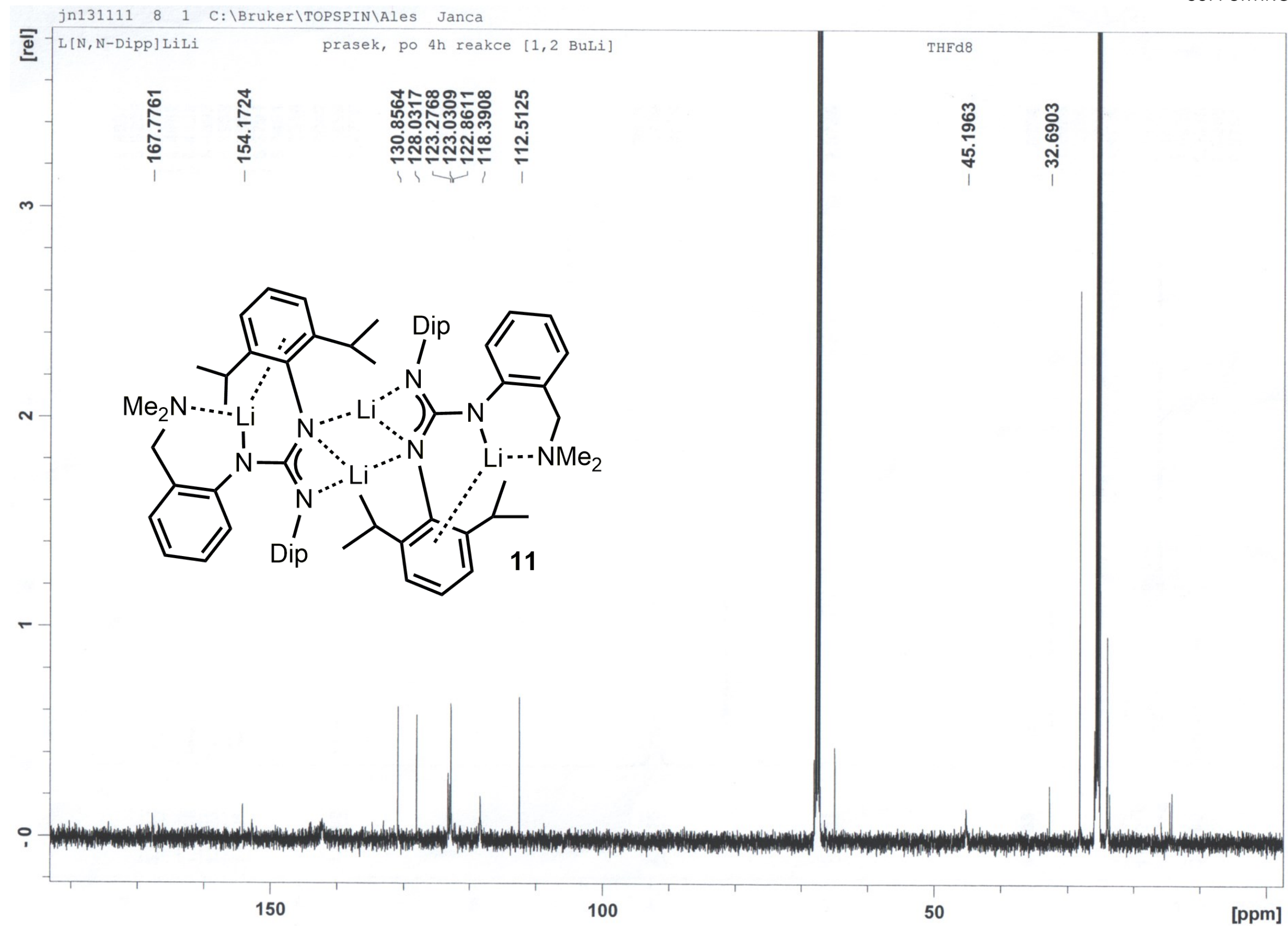




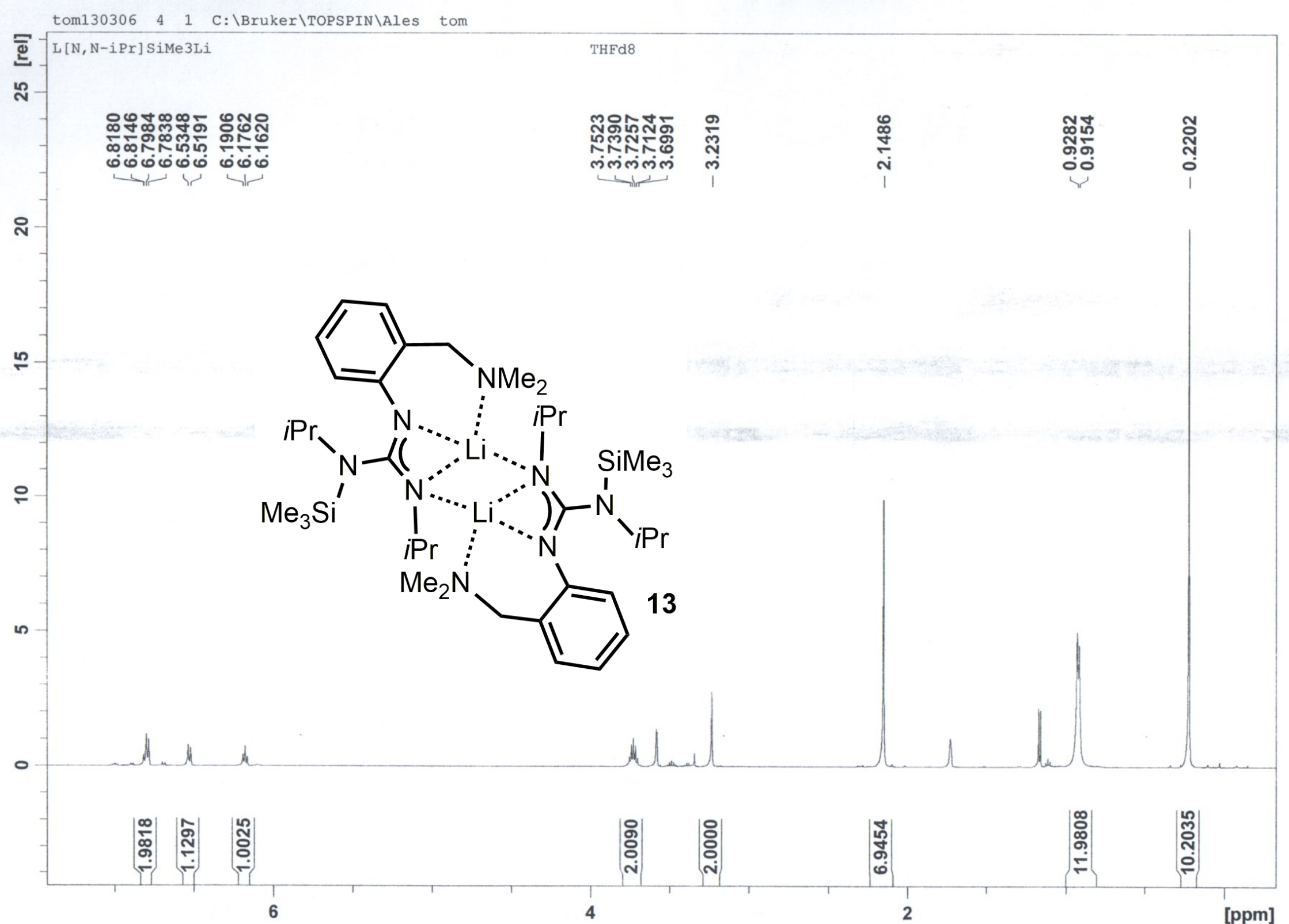




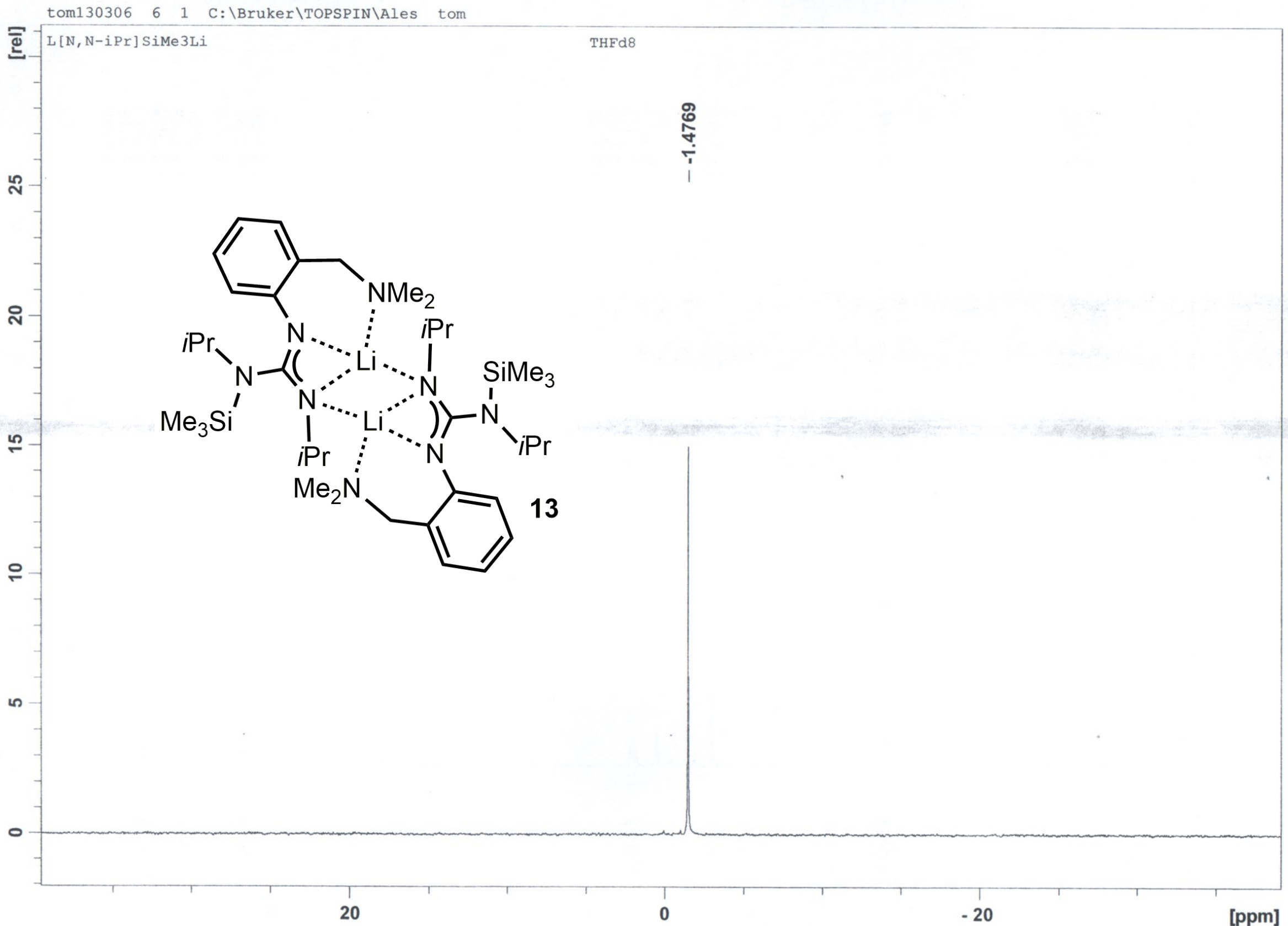




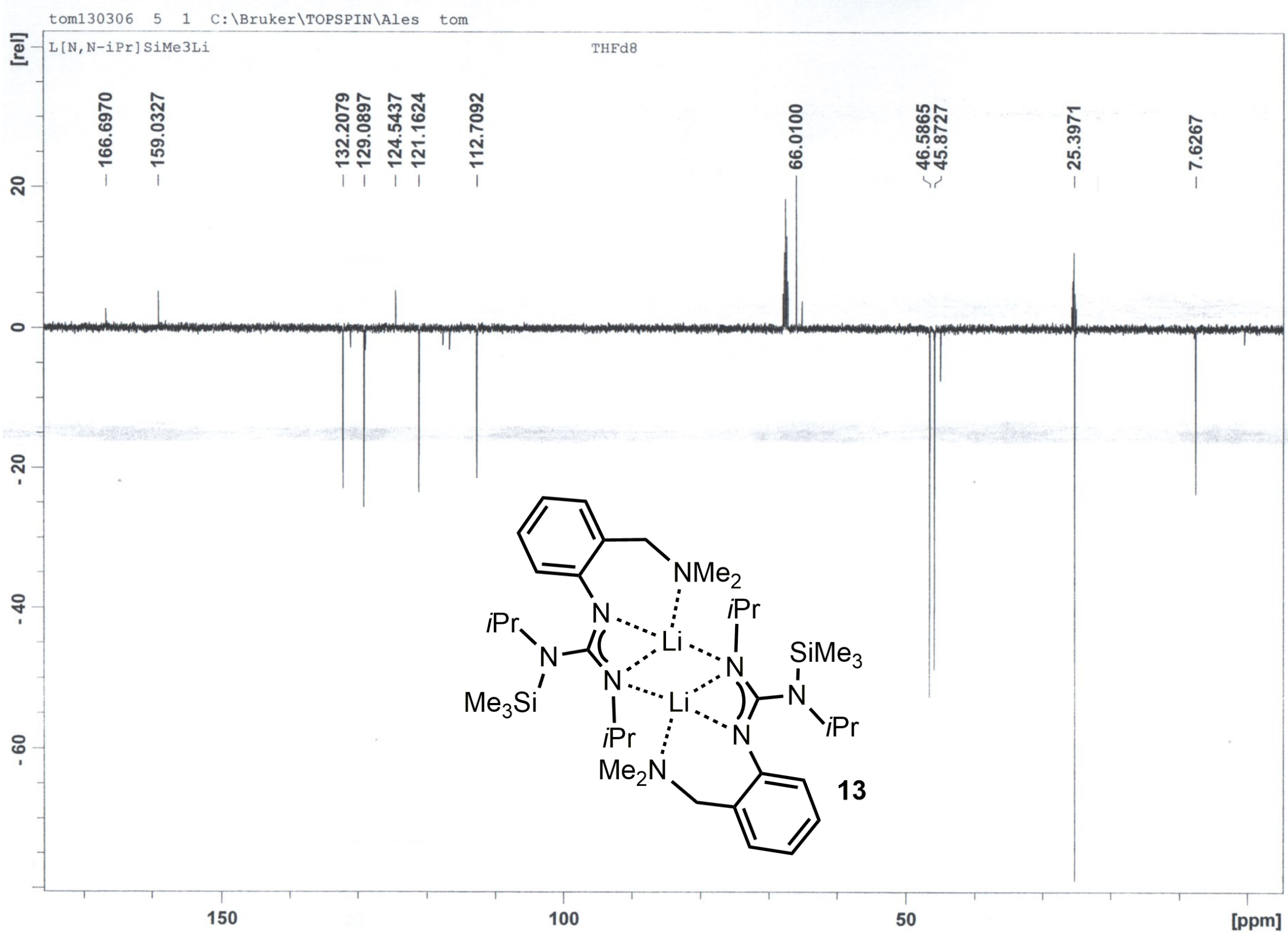




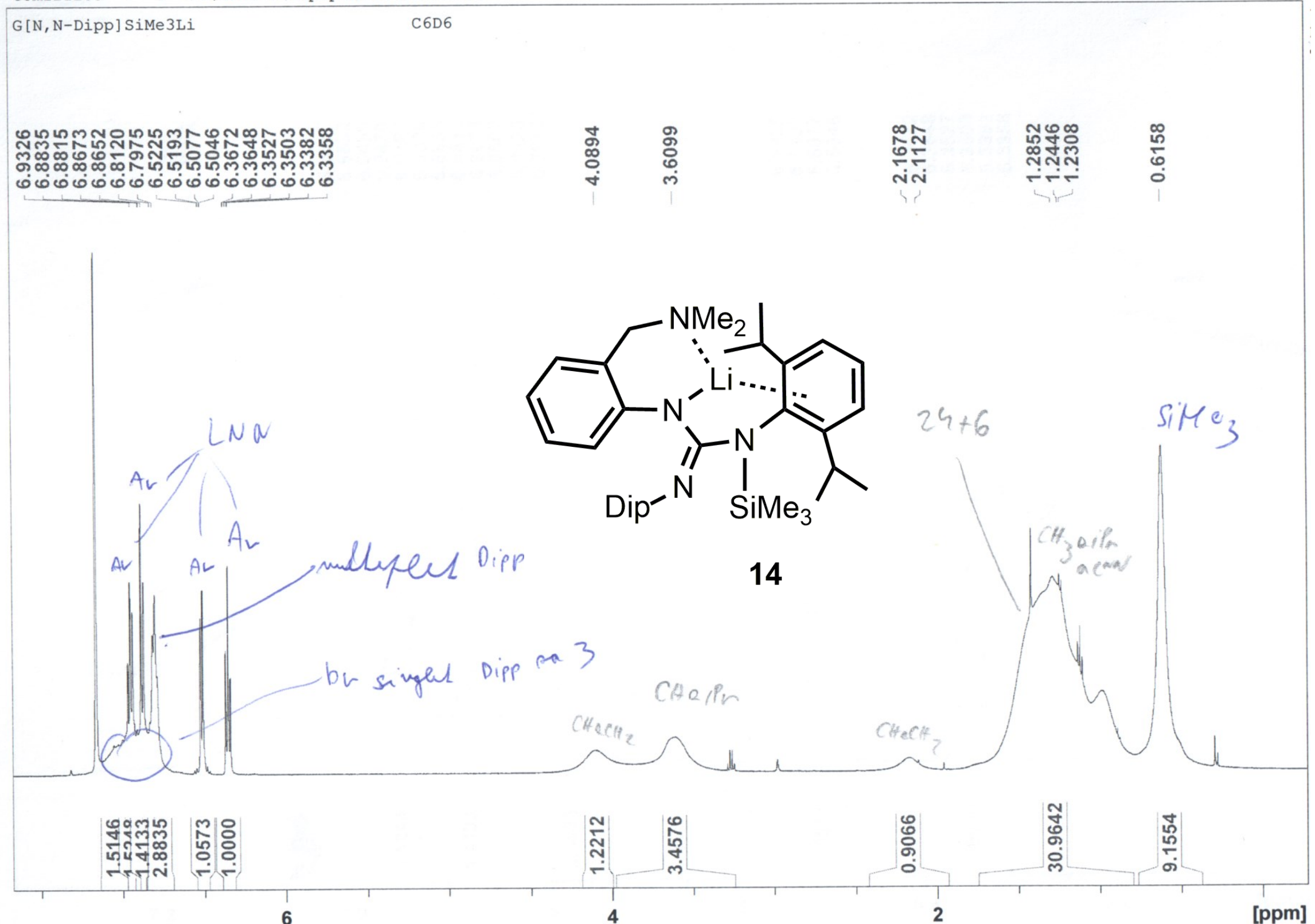




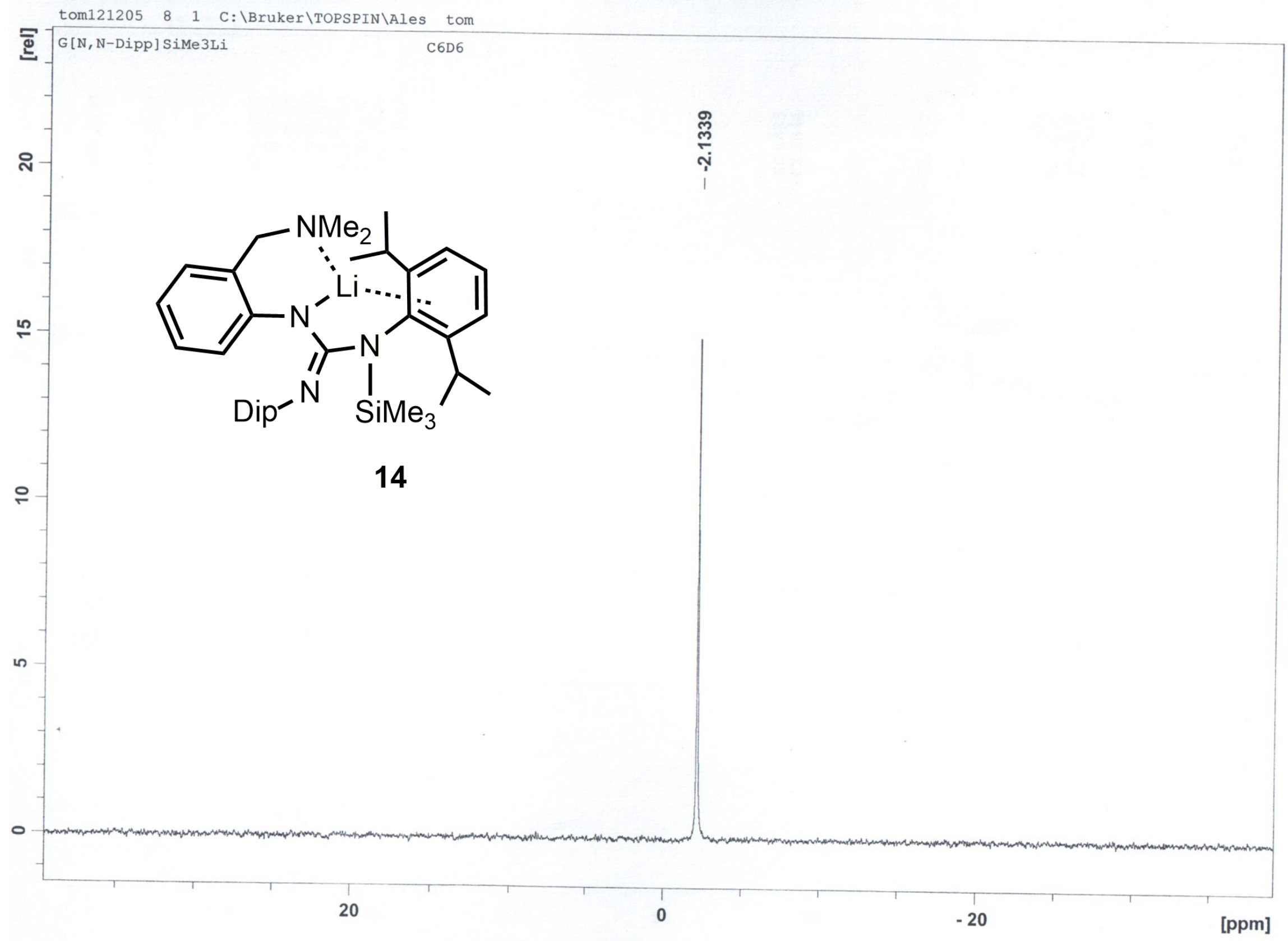




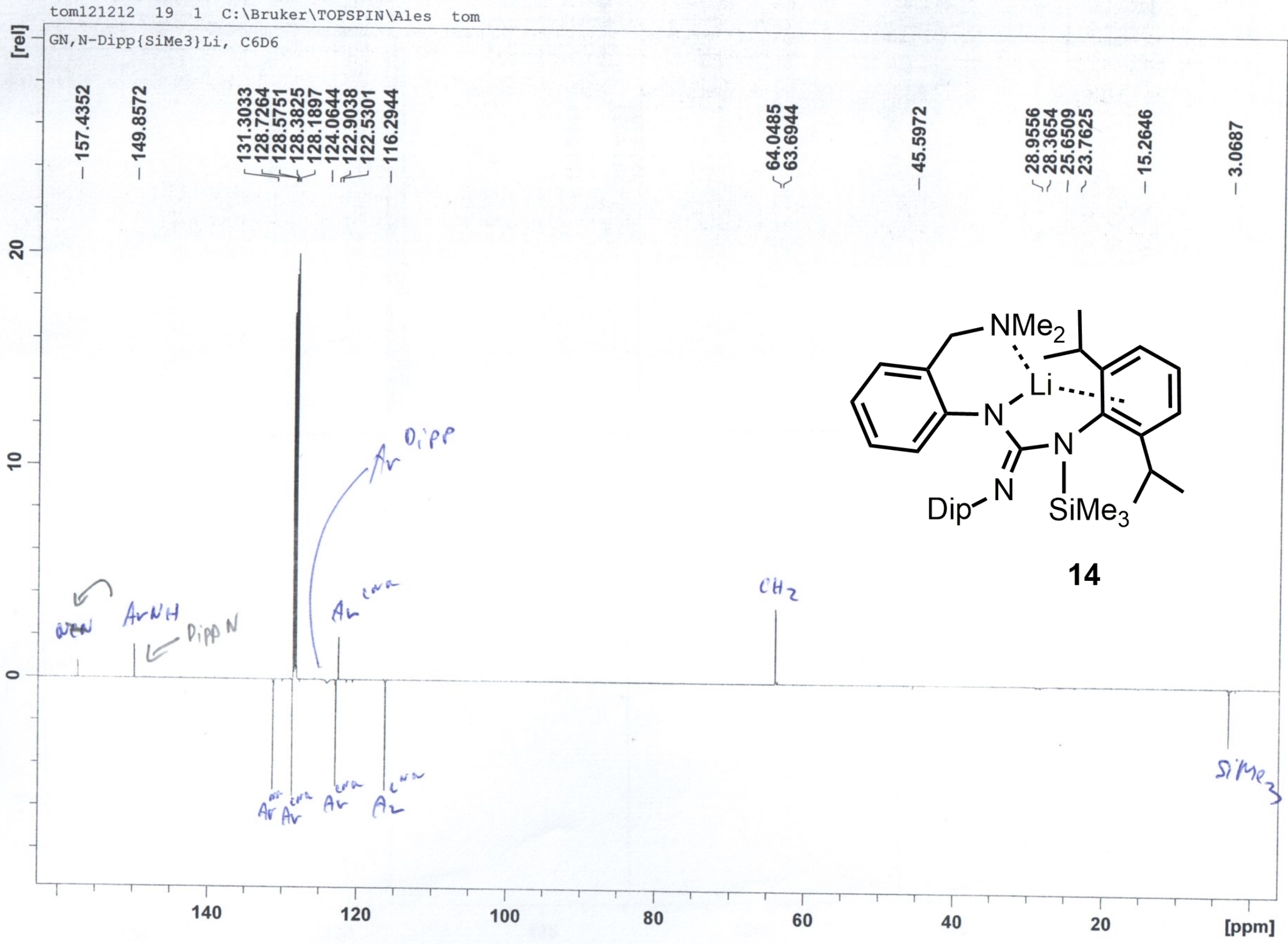




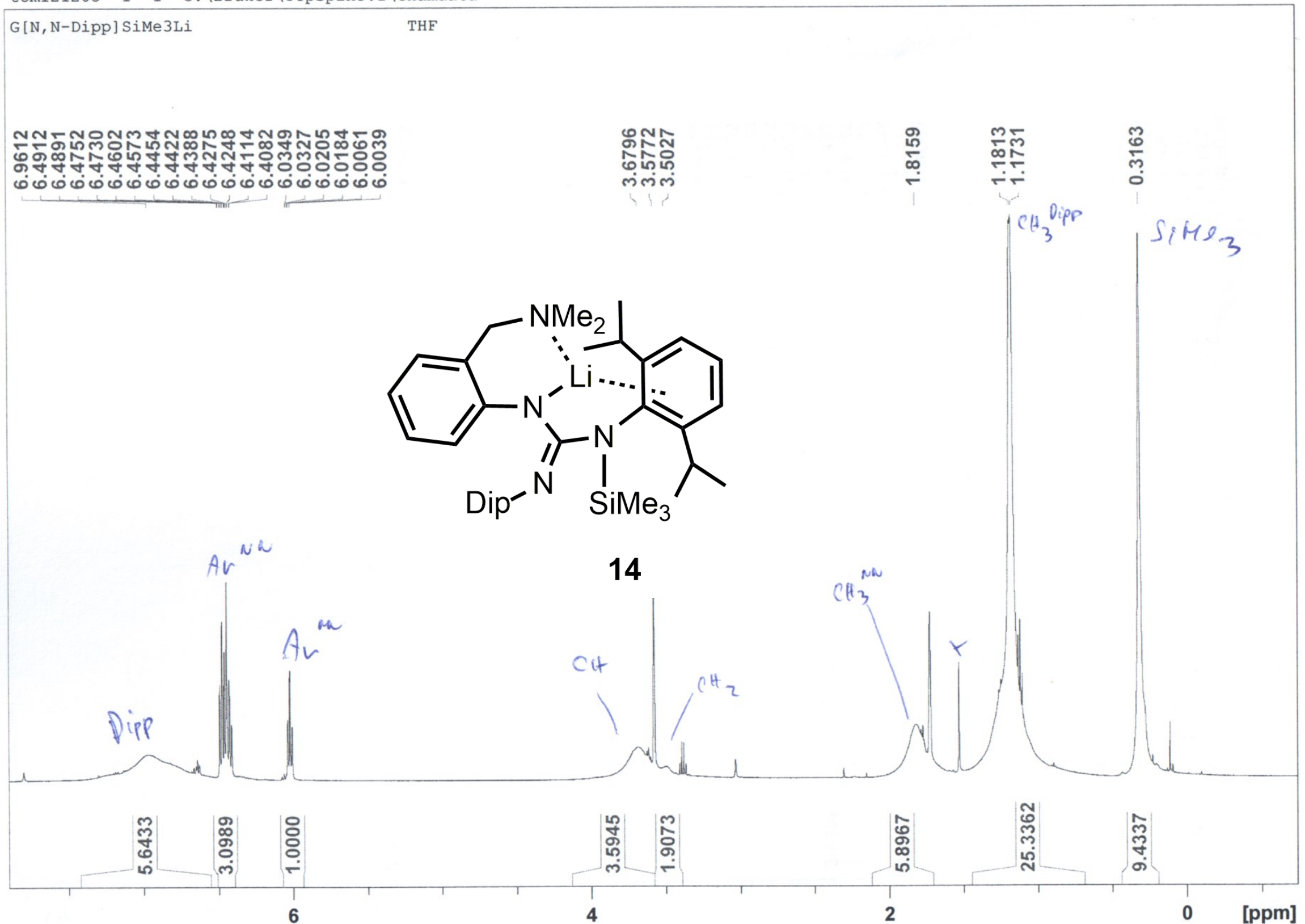




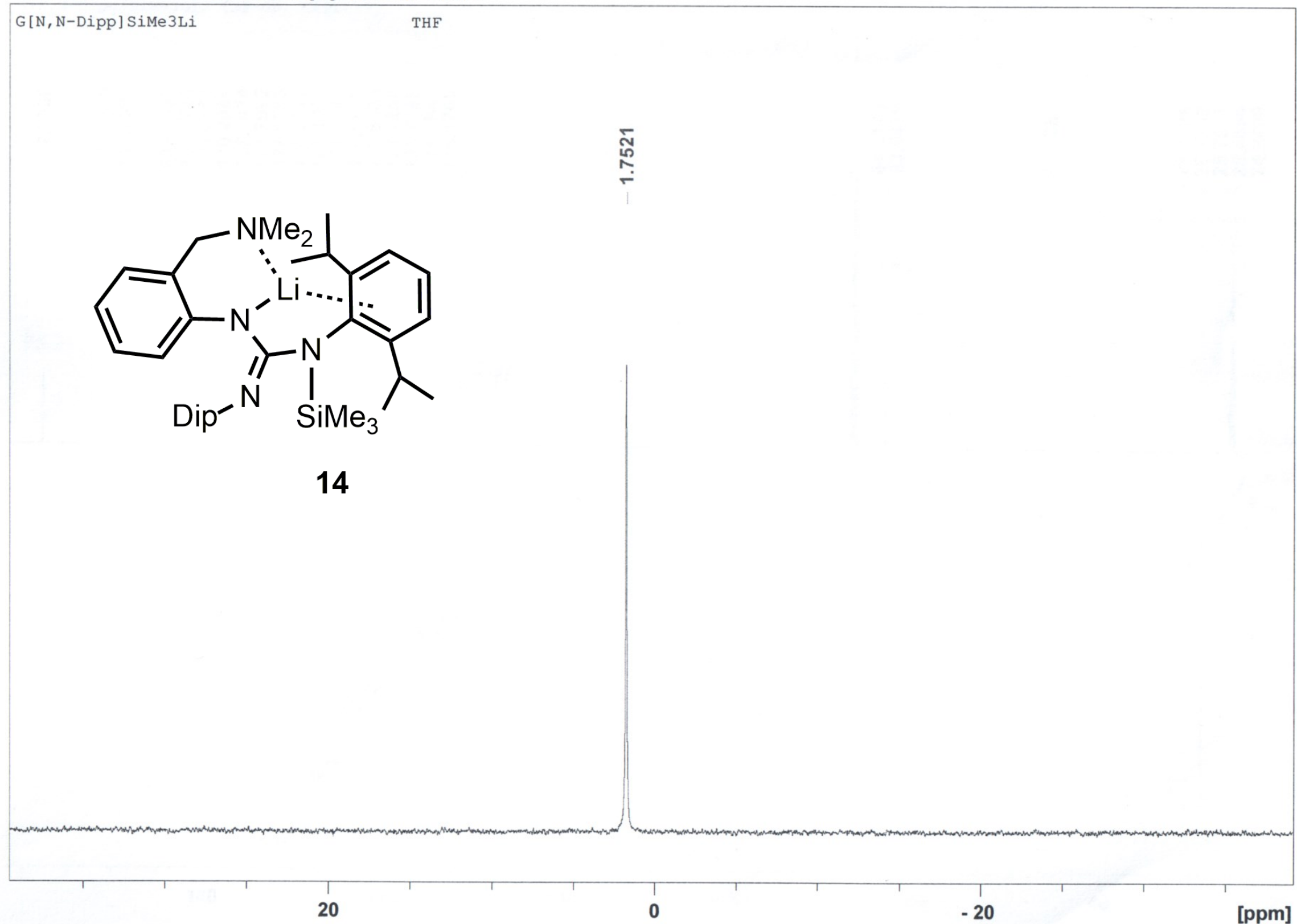




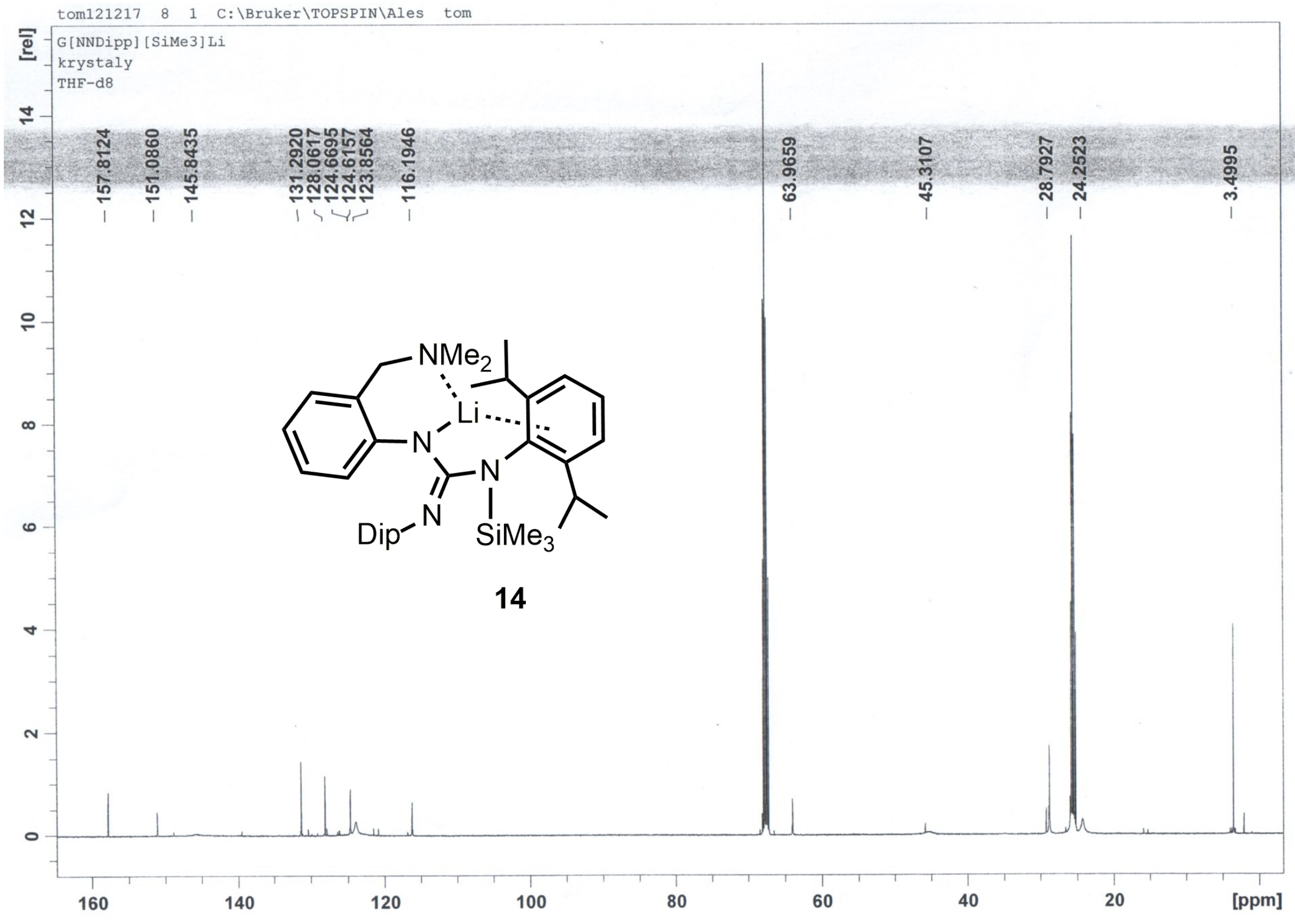




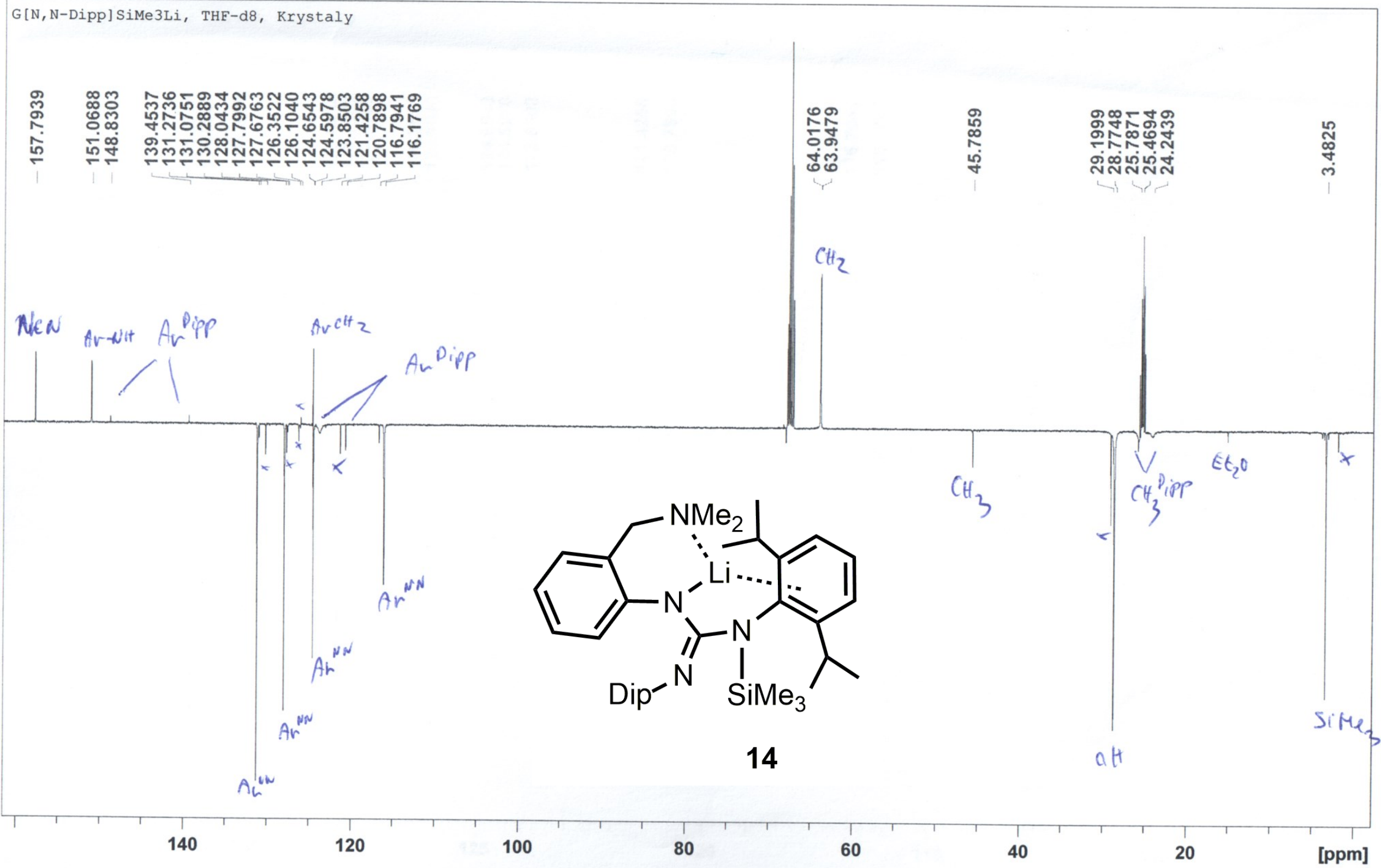

